\title{
Dust polarized emission observations of NGC 6334
}

\section{BISTRO reveals the details of the complex but organized magnetic field structure of the high-mass star-forming hub-filament network}

D. Arzoumanian ${ }^{1,2 \star}$, R. Furuya ${ }^{3,4}$, T. Hasegawa ${ }^{5}$, M. Tahani ${ }^{6}$, S. Sadavoy ${ }^{7}$, C. L. H. Hull ${ }^{8,9,10}$, D. Johnstone ${ }^{11,12}$, P. M. Koch ${ }^{13}$, S.-i. Inutsuka ${ }^{2}$, Y. Doi $^{14}$, T. Hoang ${ }^{15,16}$, T. Onaka ${ }^{17,18}$, K. Iwasaki ${ }^{5}$, Y. Shimajiri ${ }^{5}$, T. Inoue ${ }^{2}$, N. Peretto ${ }^{19}$, P. André ${ }^{20}$, P. Bastien ${ }^{21}$, D. Berry ${ }^{22}$, H.-R. V. Chen ${ }^{23}$, 13, J. Di Francesco $^{11,12}$, C. Eswaraiah ${ }^{24,25}$, L. Fanciullo ${ }^{13}$, L. M. Fissel ${ }^{7}$, J. Hwang ${ }^{15,16}$, J.-h. Kang ${ }^{15}$, G. Kim ${ }^{26}$, K.-T. Kim ${ }^{15}$, 16, F. Kirchschlager ${ }^{27}$, W. Kwon $^{28,29}$, C. W. Lee ${ }^{15,16}$, H.-L. Liu ${ }^{30,31}$, A.-R. Lyo ${ }^{15}$, K. Pattle ${ }^{32}$, A. Soam ${ }^{33}$, X. Tang ${ }^{34}$, A. Whitworth ${ }^{19}$, T.-C. Ching ${ }^{24,25}$, S. Coudé33, J.-W. Wang $^{13}$, D. Ward-Thompson ${ }^{35}$, S.-P. Lai ${ }^{23,13}$, K. Qiu ${ }^{36,37}$, T. L. Bourke ${ }^{38,39}$, D.-Y. Byun ${ }^{15,16}$, M. Chen ${ }^{12}$, Z. Chen ${ }^{40}$, W. P. Chen ${ }^{41}$, J. Cho ${ }^{42}$, Y. Choi $^{15}$, M. Choi ${ }^{15}$, A. Chrysostomou ${ }^{43,38}$, E. J. Chung ${ }^{42}$, S. Dai ${ }^{25}$, P. N. Diep ${ }^{44}$, H.-Y. Duan ${ }^{23}$, Y. Duan ${ }^{25}$, D. Eden ${ }^{45}$, J. Fiege ${ }^{46}$, E. Franzmann ${ }^{46}$, P. Friberg ${ }^{22}$, G. Fuller ${ }^{47}$, T. Gledhill ${ }^{43}$, S. Graves ${ }^{22}$, J. Greaves ${ }^{19}$, M. Griffin ${ }^{19}$, Q. Gu ${ }^{48}$, I. Han ${ }^{15,16}$, J. Hatchell ${ }^{49}$, S. Hayashi ${ }^{50}$, M. Houde ${ }^{51}$, I.-G. Jeong $^{15}$, M. Kang ${ }^{15}$, S.-j. Kang ${ }^{15}$, A. Kataoka ${ }^{5}$, K. Kawabata ${ }^{52,53,54}$, F. Kemper ${ }^{55,13}$, M.-R. Kim ${ }^{15}$, K. H. Kim ${ }^{15}$, J. Kim ${ }^{15}, 16$, S. Kim ${ }^{15,16}$, J. Kirk ${ }^{35}$, M. I.N. Kobayashi ${ }^{56}$, V. Konyves ${ }^{35}$, T. Kusune ${ }^{5}$, J. Kwon ${ }^{18}$, K. Lacaille ${ }^{57,58}$, C.-Y. Law ${ }^{48,59}$, C.-F. Lee ${ }^{13}$, Y.-H. Lee ${ }^{60,22}$, S.-S. Lee ${ }^{15,16}$, H. Lee ${ }^{42}$, J.-E. Lee ${ }^{60}$, H.-b. Li ${ }^{48}$, D. Li ${ }^{61}$, D. Li ${ }^{62}$, J. Liu ${ }^{63,64}$, T. Liu ${ }^{65}$, S.-Y. Liu ${ }^{13}$, X. Lu ${ }^{5}$, S. Mairs ${ }^{22}$, M. Matsumura ${ }^{66}$, B. Matthews ${ }^{11,12}$, G.

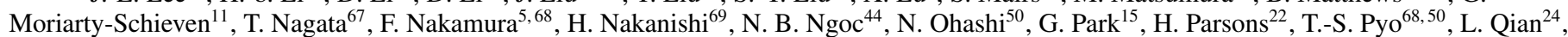
R. Rao $^{13}$, J. Rawlings ${ }^{27}$, M. Rawlings ${ }^{22}$, B. Retter ${ }^{19}$, J. Richer ${ }^{70,71}$, A. Rigby ${ }^{19}$, H. Saito ${ }^{72}$, G. Savini ${ }^{27}$, A. Scaife ${ }^{47}$, M. Seta ${ }^{73}$, H. Shinnaga ${ }^{69}$, M. Tamura $^{5,18,74}$, Y.-W. Tang ${ }^{13}$, K. Tomisaka ${ }^{5,68}$, L. N. Tram ${ }^{75}$, Y. Tsukamoto ${ }^{69}$, S. Viti ${ }^{27}$, H. Wang ${ }^{40}$, J. Xie ${ }^{25}$, H.-W. Yen $^{13}$, H. Yoo $^{15}$, J. Yuan $^{25}$, H.-S. Yun ${ }^{60}$, T. Zenko ${ }^{67}$, G. Zhang ${ }^{24}$, C.-P. Zhang ${ }^{25,24}$, Y. Zhang ${ }^{48}$, J. Zhou ${ }^{62}$, L. Zhu ${ }^{24}$, I. de Looze ${ }^{27}$, C. D. Dowell ${ }^{76}$, S. Eyres ${ }^{77}$, S. Falle ${ }^{78}$, R. Friesen $^{79}$, J.-F. Robitaille ${ }^{80}$, and S. van Loo $^{81}$

(Affiliations can be found after the references)

December 25, 2020

\section{ABSTRACT}

Context. Molecular filaments and hubs have received special attention recently thanks to new studies showing their key role in star formation. While the (column) density and velocity structures of both filaments and hubs have been carefully studied, their magnetic field (B-field) properties are not yet characterized. Consequently, the role of B-fields in the formation and evolution of hub-filament systems is not well constrained.

Aims. We aim to understand the role of the B-field and its interplay with turbulence and gravity in the dynamical evolution of the NGC 6334 filament network that harbours cluster-forming hubs and high-mass star formation.

Methods. We present new observations of the dust polarized emission at $850 \mu \mathrm{m}$ towards the $2 \mathrm{pc} \times 10 \mathrm{pc}$ map of NGC 6334 at a spatial resolution of $0.09 \mathrm{pc}$ obtained with the JCMT/SCUBA-2/POL-2 as part of the BISTRO survey. We study the distribution and dispersion of the polarized intensity $(P I)$, the polarization fraction $(P F)$, and the plane-of-the-sky (POS) B-field angle $\left(\chi_{\mathrm{B} \text { pos }}\right)$ towards the whole region, along the 10 pc-long ridge, and along the sub-filaments connected to the ridge and the hubs. We derive the power spectrum of the intensity and $\chi_{\mathrm{B}_{\mathrm{POS}}}$ along the ridge crest and compare with results obtained from simulated filaments.

Results. The observations span $\sim 3$ orders of magnitude in $I$ and $P I$ and $\sim 2$ orders of magnitude in $P F$ (from $\sim 0.2 \%$ to $\sim 20 \%$ ). A large scatter in $P I$ and $P F$ is observed for a given value of $I$. Our analyses show a complex B-field structure when observed over the whole region $(\sim 10 \mathrm{pc})$, however, at smaller scales $(\sim 1 \mathrm{pc}), \chi_{\mathrm{B}}$ vos varies coherently along the crests of the filament network. The observed power spectrum of $\chi_{\mathrm{B}}{ }_{\mathrm{POS}}$ can be well represented with a power law function with a slope $-1.33 \pm 0.23$, which is $\sim 20 \%$ shallower than that of $I$. We find that this result is compatible with the properties of simulated filaments and may indicate the physical processes at play in the formation and evolution of star-forming filaments. Along the sub-filaments, $\chi_{\mathrm{B} \text { pos }}$ rotates from being mostly perpendicular (or randomly orientated with respect) to the crests to mostly parallel as the sub-filaments merge with the ridge and hubs. This variation of the B-field structure along the sub-filaments may be tracing local velocity flows of matter in-falling onto the ridge and hubs. Our analysis also suggests a variation of the energy balance along the crests of these sub-filaments, from magnetically critical/supercritical at their far ends to magnetically subcritical near the ridge and hubs. We also detect an increase of $P F$ towards the high-column density $\left(N_{\mathrm{H}_{2}} \gtrsim 10^{23} \mathrm{~cm}^{-2}\right)$ star cluster-forming hubs that may be explained by the increase of grain alignment efficiency due to stellar radiation from the newborn stars, combined with an ordered B-field structure.

Conclusions. These observational results reveal for the first time the characteristics of the small scale (down to $\sim 0.1 \mathrm{pc}$ ) B-field structure of a $10 \mathrm{pc}$-long hub-filament system. Our analyses show variations of the polarization properties along the sub-filaments that may be tracing the evolution of their physical properties during their interaction with the ridge and hubs. We also detect an impact of feedback from young high-mass stars on the local B-field structure and the polarization properties, that could put constraints on possible models for dust grain alignment and provide important hints on the interplay between the star formation activity and interstellar B-fields.

Key words. stars: formation - ISM: clouds - ISM: structure - submillimeter: ISM - Dust polarization - Magnetic field structure

\section{Introduction}

In the last decade, observations tracing the thermal emission from the cold dust (e.g., with Herschel and Planck) and the

* Doris.Arzoumaniandastro.up.pt molecular gas of the interstellar medium (ISM) unveiled its highly filamentary structure (e.g., André et al. 2014; Molinari et al. 2010; Wang et al. 2015; Planck Collaboration Int. XXXII 2016; Hacar et al. 2018). Moreover, in star forming regions, prestellar cores and protostars are mostly observed along the 
sample of filaments that are gravitationally unstable (e.g., André et al. 2010; Könyves et al. 2015; Hacar et al. 2013). In addition, detailed analyses of the properties of these filaments and their cores indicate that a relatively small fraction $(\sim 15-20 \%)$ of the mass of the filaments is in the form of cores, and the mass distribution of these cores (CMF) has a shape similar to the initial mass function (IMF) of stars (e.g., André et al. 2010; Könyves et al. 2015, 2020).

While we still have to confirm whether or not the full mass spectrum of stars can be formed by the same physical process, these observational results suggest that fragmentation within self-gravitating filaments is the dominant mode for star formation (at least for the low- to intermediate-mass stars, e.g., André et al. 2014; Tafalla \& Hacar 2015). If this is the case, the mechanisms responsible for filament formation and fragmentation should explain the observed physical properties of the cores (and eventually that of the stars), e.g., spacing/clustering, mass functions (CMF/IMF), size distribution, angular momentum. Such a filament paradigm should also explain the global properties of the star formation process on the scales of galaxies, e.g., star formation efficiency and rate, (dense) gas depletion timescales. It is thus crucial to describe the properties and understand the dynamics of these filaments, which give the initial conditions of core/star formation and may be a main element to understand the global star formation properties on the scale of galaxies.

Observations suggest that, in star-forming molecular clouds, filaments are rarely isolated, but mostly organized in systems. We can divide these systems into two major groups:

1) The "ridge-dominated" group, which can be described by a single dense self-gravitating "main-filament" or "ridge" with a line mass $M_{\text {line }}$ larger than $M_{\text {line,crit }}$, the thermal critical value for equilibrium ${ }^{1}$. These filaments are gravitationally unstable and are observed to be forming chains of stars along their crests and are often connected from the side to lower-density "subfilaments" (e.g., Hill et al. 2011; Schneider et al. 2010; Hennemann et al. 2012; Palmeirim et al. 2013; Kirk et al. 2013a; Arzoumanian et al. 2017).

2) The "hub-dominated" group, composed by several starforming (mostly high-density) filaments merging into a highdensity "hub" (e.g., Myers 2009; Peretto et al. 2013, 2014; Williams et al. 2018). These hubs have also been identified to host young star-clusters, suggesting the important role of hubfilament configurations in star-cluster formation (e.g., Schneider et al. 2012; Kumar et al. 2020).

The formation of these ridge and hub systems is still under debate. Most observational work has focused on their (column) densities and velocity structures (e.g., Hennemann et al. 2012; Kirk et al. 2013b; Peretto et al. 2013), which have revealed velocity gradients along lower-density sub-filaments that may be feeding material to the ridges and/or hubs (e.g., Schneider et al. 2010; Palmeirim et al. 2013; Peretto et al. 2014). One remaining question, however, is the role of magnetic fields (B-fields) in hindering or supporting the flow of material. The relative role of B-fields with respect to both turbulence and gravity is not well constrained (e.g., Crutcher 2012; Hennebelle \& Inutsuka 2019).

B-fields are most often characterized via polarization measurements, assuming that interstellar dust grains are aspherical and that their major axes align perpendicular to the local

\footnotetext{
1 These filaments are thermally supercritical with line masses $\left(M_{\text {line }}\right)$ larger than the critical equilibrium value for isothermal cylinders (e.g. Inutsuka \& Miyama 1997), $M_{\text {line,crit }}^{T}=2 c_{\mathrm{s}}^{2} / G \sim 16.5 \mathrm{M}_{\odot} / \mathrm{pc}$ (Ostriker 1964), where $c_{\mathrm{s}} \sim 0.2 \mathrm{~km} \mathrm{~s}^{-1}$ is the sound speed for $T \sim 10 \mathrm{~K}$.
}

B-field orientation (e.g., Lee \& Draine 1985; Lazarian et al. 1997; Hildebrand et al. 2000; Andersson et al. 2015; Lazarian et al. 2015). Consequently, a fraction of the thermal emission from these dust grains will be linearly polarized relative to the direction of the plane-of-sky (POS) B-field (e.g., Jones \& Spitzer 1967; Hildebrand 1983; Andersson 2015). All-sky dust polarized emission of the ISM observed by Planck at $850 \mu \mathrm{m}$ has revealed organized B-fields on large scales $(>1-10 \mathrm{pc}$, see, e.g., Planck Collaboration Int. XIX 2015). Statistical analysis of the relative orientation between the filaments and the plane-of-the-sky (POS) B-field angle shows that low column density filaments are mostly parallel to the local B-field while high column density filaments tend to be perpendicular to the B-field lines (Planck Collaboration Int. XXXII 2016). Similar results are also inferred from infrared polarization data (e.g., Sugitani et al. 2011; Palmeirim et al. 2013; Cox et al. 2016; Soler et al. 2016). Comparisons of these observations with numerical simulations suggest that B-fields play a dynamically important role in the formation and evolution of filaments in a mostly sub/trans-Alfvénic turbulent ISM (Falceta-Gonçalves et al. 2008, 2009; Soler et al. 2013; Planck Collaboration Int. XXXV 2016). There is also an indication that the orientation of the B-field towards dense filaments changes from being nearly perpendicular in the surrounding cloud to more parallel in the filament interior (Planck Collaboration Int. XXXIII 2016). However, the low resolution of Planck data $\left(\sim 5^{\prime}-10^{\prime}\right.$ or $\sim 0.2-0.6 \mathrm{pc}$ at distance $\lesssim 500 \mathrm{pc}$ or $>0.5 \mathrm{pc}$ at distances $>1 \mathrm{kpc}$ ) is insufficient to resolve the B-field structure at the characteristic $\sim 0.1 \mathrm{pc}$ transverse size of molecular filaments (Arzoumanian et al. 2011, 2019), which is also the scale at which filament fragmentation and core formation occurs (Tafalla \& Hacar 2015; Kainulainen et al. 2017; Shimajiri et al. 2019). Consequently, the geometry of the B-field within filaments and its effects on fragmentation and star formation are mostly unknown.

To gain insight into the B-field structure along dense filaments and improve our understanding of the role of the magnetic field in the star formation process, we analyze new $850 \mu \mathrm{m}$ data obtained towards the NGC 6334 star-forming filamentary region observed as part of the B-field In STar-forming Region Observations (BISTRO) using SCUBA-2/POL-2 installed on the James Clerk Maxwell Telescope (JCMT). While the original BISTRO survey was designed to cover a variety of nearby star-forming regions, with a focus on the Gould Belt molecular clouds (Ward-Thompson et al. 2017), the NGC 6334 field is part of BISTRO-2, a follow-up BISTRO survey, which is an extension aiming at observing mostly high-mass star-forming regions.

NGC 6334 is a high-mass star-forming complex that lies within the Galactic plane at a relatively nearby distance of $1.3 \pm 0.3 \mathrm{kpc}$ (Chibueze et al. 2014). This region has been the target of multiple studies at different wavelengths (see Persi \& Tapia 2008, for an extensive review). At optical wavelengths, NGC 6334 is seen as a grouping of well-documented H II regions also known as the "Cat's Paw" (GUM61, GUM 62, GUM 63, GUM 64, H II 351.2+0.5, and GM1-24, Persi \& Tapia 2010; Russeil et al. 2016). In between these H II bubbles, along the Northeast-Southwest direction lies a 10 pc-long filamentary cloud that is very bright at (sub)millimeter wavelengths (Kraemer \& Jackson 1999; Matthews et al. 2008; Russeil et al. 2013; Zernickel et al. 2013; Tigé et al. 2017). This filamentary cloud is dominated by both a dense ridge threaded by sub-filaments, and by two hub-like structures towards its Northeast end (cf. Fig. 1). This ridge itself is actively forming high-mass stars as 
revealed by compact radio emission, ultra-compact $\mathrm{H}$ II regions, maser sources, and molecular outflows identified along or next to its crest (Sandell 2000; McCutcheon et al. 2000; Muñoz et al. 2007; Qiu et al. 2011). The NGC 6334 filament has a line mass $M_{\text {line }} \sim 1000 \mathrm{M}_{\odot} / \mathrm{pc}\left(>M_{\text {line,crit }}\right)$ with column densities $N_{\mathrm{H}_{2}} \gtrsim 10^{23} \mathrm{~cm}^{-2}$ over most of its $10 \mathrm{pc}$ long crest (André et al. 2016) and it is fragmented into a series of relatively massive cores with a mean mass $\sim 10 \mathrm{M}_{\odot}$ (e.g., Shimajiri et al. 2019). The filament inner width is observed to be of the order of $0.1 \mathrm{pc}$ (André et al. 2016), compatible with the findings derived from statistical analysis of dust continuum Herschel observations of nearby and less massive filaments (Arzoumanian et al. 2011, 2019; Koch \& Rosolowsky 2015). This similarity suggests that the formation process of stars from low to high-mass stars may be similar, and gravitational fragmentation of $\sim 0.1 \mathrm{pc}$-wide filaments may be also the main mode of intermediate- to high-mass star formation (Shimajiri et al. 2019; André et al. 2019).

The magnetic field structure of NGC 6334 has been investigated at large scales $(\sim 10 \mathrm{pc})$ using starlight polarization and at small scales $(\lesssim 0.1 \mathrm{pc})$ towards the dense cores using interferometric observations of dust polarized emission with the SMA (Li et al. 2006; Zhang et al. 2014; Li et al. 2015; Juárez et al. 2017). By combining and interpolating some of the latter measurements, Li et al. (2015) suggested that the orientation of the B-field does not change significantly over the various scales, pointing to the important dynamical role played by the B-field in the formation of NGC 6334. In this paper, we study the Bfield structure and the polarization properties towards the NGC 6334 high-mass star-forming region at scales ranging from about $0.1 \mathrm{pc}$ to $10 \mathrm{pc}$, using the dust polarized emission at $850 \mu \mathrm{m}$ observed as part of the BISTRO survey. We also present the Bfield structure observed at lower resolution by Planck around the NGC 6334 region up to scales of $\sim 40 \mathrm{pc}$. We further compare the characteristics derived from this high-mass star-forming region with those inferred from the analysis of molecular clouds forming lower mass stars. This paper is organized as follows: In Sect. 2, we describe the observations and the data reduction. In Sect. 3, we present the spatial distribution of the observed polarized emission and scatter plots relating the different polarization parameters. In Sect. 4, we analyse the polarization and physical properties of different crests identified in the filament network. In Sect. 5, we present a power spectrum analysis of the observed emission (total intensity and B-field structure) along the filament crest. In Sect. 6, we discuss the possible physical origin of the observed polarization properties and the B-field structure of the high-mass star-forming hub-filament system. We give a summary of the analysis and results in Sect. 7.

\section{Observations}

\subsection{SCUBA-2/POL-2 $850 \mu \mathrm{m}$ BISTRO observations}

The total and polarized dust thermal continuum emission towards NGC 6334 was observed using SCUBA-2/POL-2 (Bastien et al. 2011; Holland et al. 2013; Friberg et al. 2016) installed on the JCMT between August 2017 and April 2019 (project code M17BL011). The observations were carried out under dry weather conditions with the atmospheric opacity at $225 \mathrm{GHz}$ ranging between 0.03 and 0.07 . The observations were done with the standard SCUBA-2/POL-2 DAISY mapping mode with a constant scanning speed of $4^{\prime \prime} \mathrm{s}^{-1}$ (POL2-DAISY) and a data sampling rate of $8 \mathrm{~Hz}$. Two maps of about $12^{\prime}$ in diameter each were taken towards the North and South of the elongated filament to cover the entire $10 \mathrm{pc}-$ long structure. A total of 30 hours on-source were needed to observe the two fields (with 20 exposures per field). The North and South fields were centred on $(17: 20: 50.011,-35: 45: 34.33)$ and $(17: 20: 19.902,-35: 54: 30.45)$ in (R.A. J2000, Dec. J2000), respectively (see Fig. A.1 for the limits of the two observed DAISY sub-fields). The two sub-fields were then combined to create the mosaicked $\sim 20^{\prime} \times 20^{\prime}$ Stokes parameter maps (Fig. 1). The flux conversion factor (FCF) of POL-2 at $850 \mu \mathrm{m}$ is taken to be $725 \mathrm{Jy} \mathrm{pW}^{-1}$ beam $^{-1}$ for the three Stokes $I, Q$, and $U$ parameters (Friberg et al. 2016). The spatial distributions of the Stokes $I, Q$, and $U$ parameters are derived from their time-series measurements using the pol $2 \mathrm{map}^{2}$ data reduction pipeline (including skyloop) that is based on the Starlink routine makemap (Chapin et al. 2013; Currie et al. 2014) and optimized for the SCUBA-2/POL-2 data (c.f., Coudé et al. 2019 , for details on the data reduction process).

The JCMT has a diameter of $15 \mathrm{~m}$ and achieves an effective half-power-beam-width (HPBW) angular resolution of 14" at $850 \mu \mathrm{m}$ (Dempsey et al. 2013; Friberg et al. 2016). The data are projected onto grid maps with pixel sizes of $4^{\prime \prime}$ and $12^{\prime \prime}(\sim$ HPBW), both at the spatial resolution of $14^{\prime \prime}$ (HPBW of the JCMT at $850 \mu \mathrm{m}$ ).

Figure 1 presents the maps of the three Stokes parameters of NGC 6334. The Stokes I map is very similar to what was observed by the previous generation bolometer on the JCMT (e.g., Matthews et al. 2008) and the APEX/ArTéMiS map at $350 \mu \mathrm{m}$ (at an angular resolution of $8^{\prime \prime}$, André et al. 2016). The Stokes $Q$ and $U$ parameter maps provide a first insight into the spatial structure of the linearly polarized emission of the cold dust grains. The spatial distribution of these two parameters is different and some abrupt changes from positive to negative can be seen. These variations are a first indication of the change of the POS B-field structure.

\subsection{Polarization parameters}

The polarization parameters are calculated from the combination of the Stokes $I, Q$, and $U$ parameters using the following relations

$$
\begin{aligned}
& P I_{\mathrm{obs}}=\sqrt{Q^{2}+U^{2}} \\
& P F_{\mathrm{obs}}=P I_{\mathrm{obs}} / I \\
& \psi=0.5 \arctan (U, Q),
\end{aligned}
$$

where $P I_{\mathrm{obs}}, P F_{\mathrm{obs}}$, and $\psi$ are the observed polarized intensity, the observed polarization fraction, and the polarization angle, respectively. The polarization angle is calculated in the IAU convention, i.e., North to East in the equatorial coordinate system. The POS magnetic field ( $\left.B_{\mathrm{POS}}\right)$ orientation is obtained by adding $90^{\circ}$ to the polarization angle

$\chi_{B_{\mathrm{POS}}}=\psi+90^{\circ}$.

Given the noise present in the observed Stokes parameters, $P I_{\mathrm{obs}}$ and $P F_{\text {obs }}$ are biased positively. We debias $P I_{\mathrm{obs}}$ and $P F_{\text {obs }}$ taking into account the uncertainties on $Q$ and $U, \delta Q$ and $\delta U$, respectively, using the relations (e.g., Vaillancourt 2006):

$$
\begin{aligned}
& P I=\sqrt{Q^{2}+U^{2}-0.5\left(\delta Q^{2}+\delta U^{2}\right)} \\
& P F=P I / I .
\end{aligned}
$$

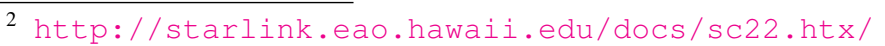
sc22. html 

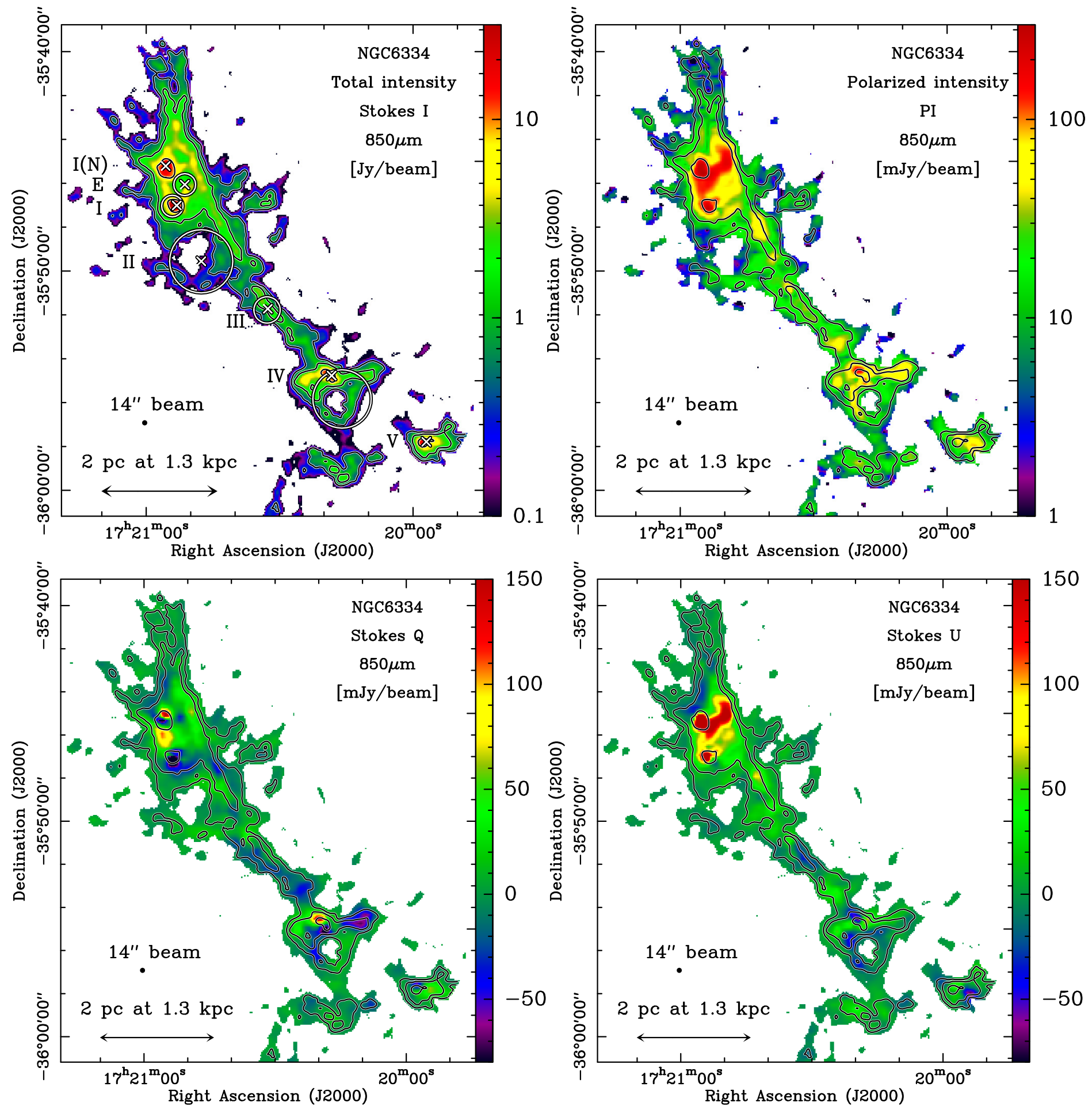

Fig. 1. Stokes parameter maps of the total $I$ (top-left) and the polarized $Q$ and $U$ (bottom-left and -right, respectively) thermal dust emission at $850 \mu \mathrm{m}$ observed with the JCMT SCUBA-2/POL-2 towards NGC 6334 as part of the BISTRO survey. The top right hand side panel shows the map of the (debiased) polarized emission, PI. The half-power-beam-width (HPBW) resolution of these maps is 14". For all maps, the plotted emission corresponds to $S N R(I)>25$. The contours, which are the same for all plots, correspond to $I=0.4,1.4$, and $8 \mathrm{Jy}_{\text {beam }}^{-1}$ or $N_{\mathrm{H}_{2}} \sim$ $\left(2,6\right.$, and 37) $\times 10^{22} \mathrm{~cm}^{-2}$. The lowest contour of $I=0.4 \mathrm{Jy}_{\text {beam }}{ }^{-1}$ is equivalent to $S N R(I) \sim 300$ and $S N R(P I) \sim 10$. The white crosses on the $I$ map indicate the seven high-mass star-forming regions numbered from I to V from North to South in addition to I(N) and E in the North of the field. The white circles show the positions and sizes of UCH II and H II regions (see Persi \& Tapia 2008, and references therein).

We calculate the uncertainties on the polarization parameters as:

$\delta P I=\sqrt{(Q \delta Q)^{2}+(U \delta U)^{2}} / P I$

$\delta P F=P F \sqrt{(\delta P I / P I)^{2}+(\delta I / I)^{2}}$

$\delta \psi=0.5 \sqrt{(U \delta Q)^{2}+(Q \delta U)^{2}} / P I^{2}$.

Article number, page 4 of 31
We estimate the signal-to-noise-ratio $(S N R)$ for each of the quantities and use the following notation in the paper: $S N R($ value $)=$ value/dvalue, where value is $I, P I$, or PF. Appendix A presents the distribution of the uncertainties of the different parameters over the observed region (Fig. A.1). The scat- 

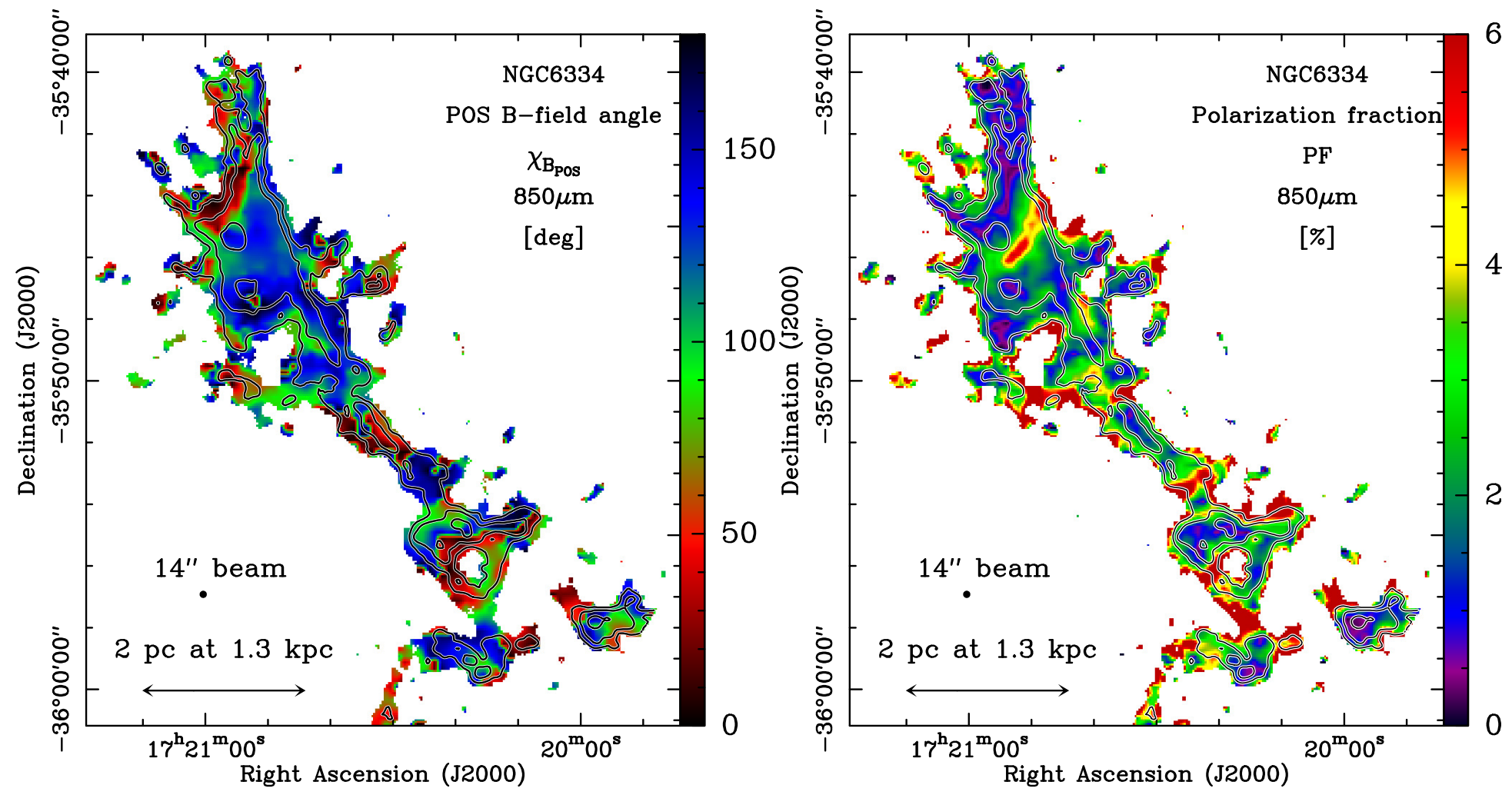

Fig. 2. Maps of the POS B-field angle $\chi_{B_{\mathrm{POS}}}($ Left $)$ and of the polarization fraction PF (Right). The HPBW resolution of the maps is $14^{\prime \prime}$. The plotted emission correspond to $S N R(I)>25$. The contours are $I=0.4,1.4$, and $8 \mathrm{Jy} \mathrm{beam}^{-1}$, as in Fig. 1 .

ter plots and histograms of Figs. A.2, A.4, and A.5 show the uncertainties of the Stokes parameters and of the polarization properties derived from the BISTRO data.

The relations we used to debias $P I$ and $P F$ (Eqs. 2 and 3) have been shown to be reliable for $S N R(P I) \gtrsim 3$ (Vaillancourt 2006; Plaszczynski et al. 2014; Montier et al. 2015; Hull \& Plambeck 2015). We thus limit our quantitative analysis for data points with $S N R(P I)>3$.

Figures 1, 2, 3, and 4 show the maps of the derived polarization properties. NGC 6334 is very bright at $850 \mu \mathrm{m}$ in both total and polarized emission. Most of the emission (corresponding to gas column density $N_{\mathrm{H}_{2}} \gtrsim 1 \times 10^{21} \mathrm{~cm}^{-2}$, see also below) is detected with $S N R(I) \gg 25$ and $S N R(P I) \gg 3$ (see Figs. A.3 and A.4). For our analysis, when not mentioned otherwise, we select data points with $S N R(I)>25$, which encompass $96 \%$ of the emission with $S N R(P I)>3$ and correspond to $\sim 1300$ independent beams towards the observed $2 \mathrm{pc} \times 10 \mathrm{pc}$ field.

\subsection{Column density and comparison with Herschel data}

We estimate the column density $\left(N_{\mathrm{H}_{2}}\right)$ from the total intensity Stokes $I$ values at $850 \mu \mathrm{m}$ with the relation $N_{\mathrm{H}_{2}}=$ $I_{850} /\left(B_{850}[T] \kappa_{850} \mu_{\mathrm{H}_{2}} m_{\mathrm{H}}\right)$, where $B_{850}$ is the Planck function, $T=$ $20 \mathrm{~K}$ is the mean dust temperature of NGC 6334 derived from Herschel data (see, Russeil et al. 2013, and Appendix B.1 for a discussion on this adopted value), $\kappa_{850}=0.0182 \mathrm{~cm}^{2} / \mathrm{g}$ is the dust opacity per unit mass of dust + gas at $850 \mu \mathrm{m}$ (e.g., Ossenkopf \& Henning 1994), $\mu_{\mathrm{H}_{2}}=2.8$ is the mean molecular weight per hydrogen molecule (e.g., Kauffmann et al. 2008), and $m_{\mathrm{H}}$ is the mass of a hydrogen atom.

In Appendix B.2, we compare the column density map derived from BISTRO $850 \mu \mathrm{m}$ data with the column density map derived from SPIRE+ArTéMiS $350 \mu \mathrm{m}$ at $T=20 \mathrm{~K}$ (presented by André et al. 2016, at a spatial resolution of $8^{\prime \prime}$ ) smoothed to the same resolution of the BISTRO 14" data. As can be seen in Fig. B.2, for column densities $\gtrsim 3 \times 10^{22} \mathrm{~cm}^{-2}$ both maps agree within a factor $<2$. Furthermore, when we subtract a "Galactic emission" of $3 \times 10^{22} \mathrm{~cm}^{-2}$ (André et al. 2016) from the SPIRE+ArTéMiS column density map, this latter map matches very well with the column density map derived from BISTRO observations at $850 \mu \mathrm{m}$ (within the dispersion of the emission towards the observed region). This "Galactic emission" of $3 \times 10^{22} \mathrm{~cm}^{-2}$ (equivalent to $124 \mathrm{MJy} / \mathrm{sr}$ ) may correspond to a combination of extended emission physically linked to the NGC 6334 molecular complex and large-scale foreground or background emission observed towards NGC 6334, which is itself located within $0.6 \mathrm{deg}$ of the Galactic Plane. As is usual with ground-based submillimeter (submm) continuum observations, the SCUBA2-POL2 observations carried out with the JCMT are affected by correlated sky noise over the entire observed field. Given that the data reduction subtracts this correlated noise, the data are significantly affected by missing flux (e.g., Sadavoy et al. 2013) at scales $\gtrsim 6^{\prime}$ larger than the field-of-view of the DAISY map with uniform noise coverage.

Comparing with the emission observed with the Herschel space telescope, which is in principle sensitive to the large scale emission, we conclude that the BISTRO data of NGC 6334 recover most of the (total intensity) flux of the dense, thin, and elongated molecular filamentary structure on which we focus our analysis in the following (c.f., Appendix B.2).

\subsection{Comparison with Planck data}

As for the total intensity (see Sect.2.3), the polarized intensity observed by the JCMT/SCUBA2-POL 2 may be affected by missing flux at large scales. To estimate this missing flux, we use 


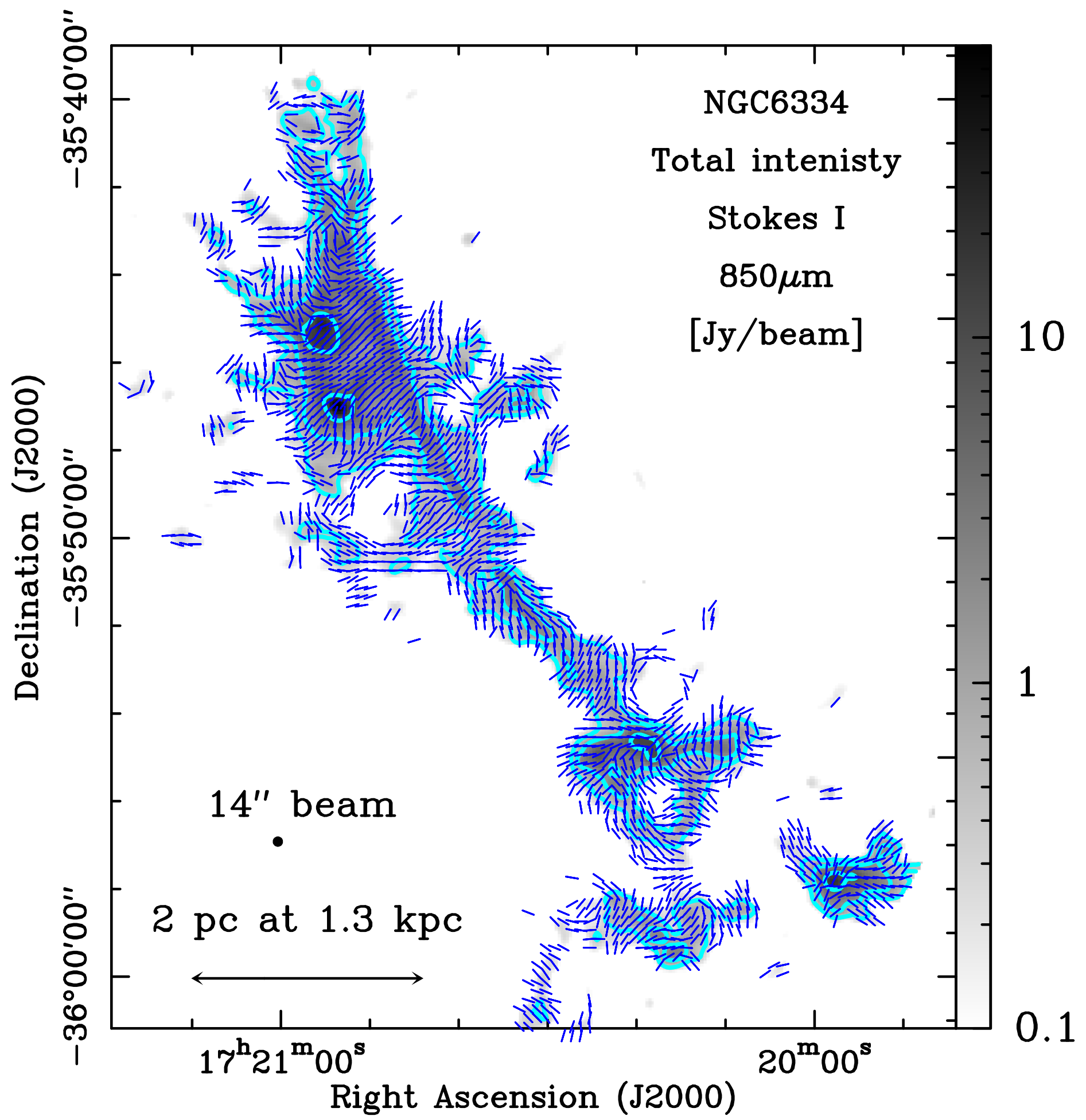

Fig. 3. Total intensity Stokes $I$ map (same as Fig. 1-top left). The blue short lines (all with the same length) show the orientation of the POS B-field angle $\left(\chi_{B_{\mathrm{POS}}}\right)$ for $S N R(I)>25$ and $S N R(P I)>3$. The contours are $I=0.4,1.4$, and $8 \mathrm{Jy}_{\text {beam }}{ }^{-1}$, as in Fig. 1.

the $850 \mu \mathrm{m}$ Planck ${ }^{3}$ polarization data at a resolution of $5^{\prime}$. From the Stokes $I^{\mathrm{p}}, Q^{\mathrm{p}}$, and $U^{\mathrm{p}}$ Planck maps, we derive the polarization properties $\chi_{B_{\mathrm{POS}}}^{\mathrm{p}}, P I^{\mathrm{p}}$, and $P F^{\mathrm{p}}$ using Eqs. 1,2, and 3, respectively. Figure 5 shows a large-scale map around NGC 6334 at a resolution of $5^{\prime}$. NGC 6334 lies within $0.6^{\circ}$ of the Galactic plane and the surrounding POS B-field is mostly observed parallel to this plane. Towards the NGC 6334 filamentary molec-

\footnotetext{
3 Planck archival data have been retrieved from http: / / www . esa. int/Planck
}

ular cloud, however, the B-field changes orientation to become mostly perpendicular to the elongated filament. This was previously reported by a study using starlight polarization ( $\mathrm{Li}$ et al. 2006). At the NGC 6334 distance of $1.3 \mathrm{kpc}$, the Planck data with a resolution of $5^{\prime}(\sim 2 \mathrm{pc}$ at $1.3 \mathrm{kpc})$ cannot resolve the Bfield structure of NGC 6334 with a transverse size $\ll 2$ pc. This small-scale $B_{\mathrm{POS}}$ structure is now revealed by BISTRO observations at $14^{\prime \prime}$ (see Figs. 3 and 4 ). 


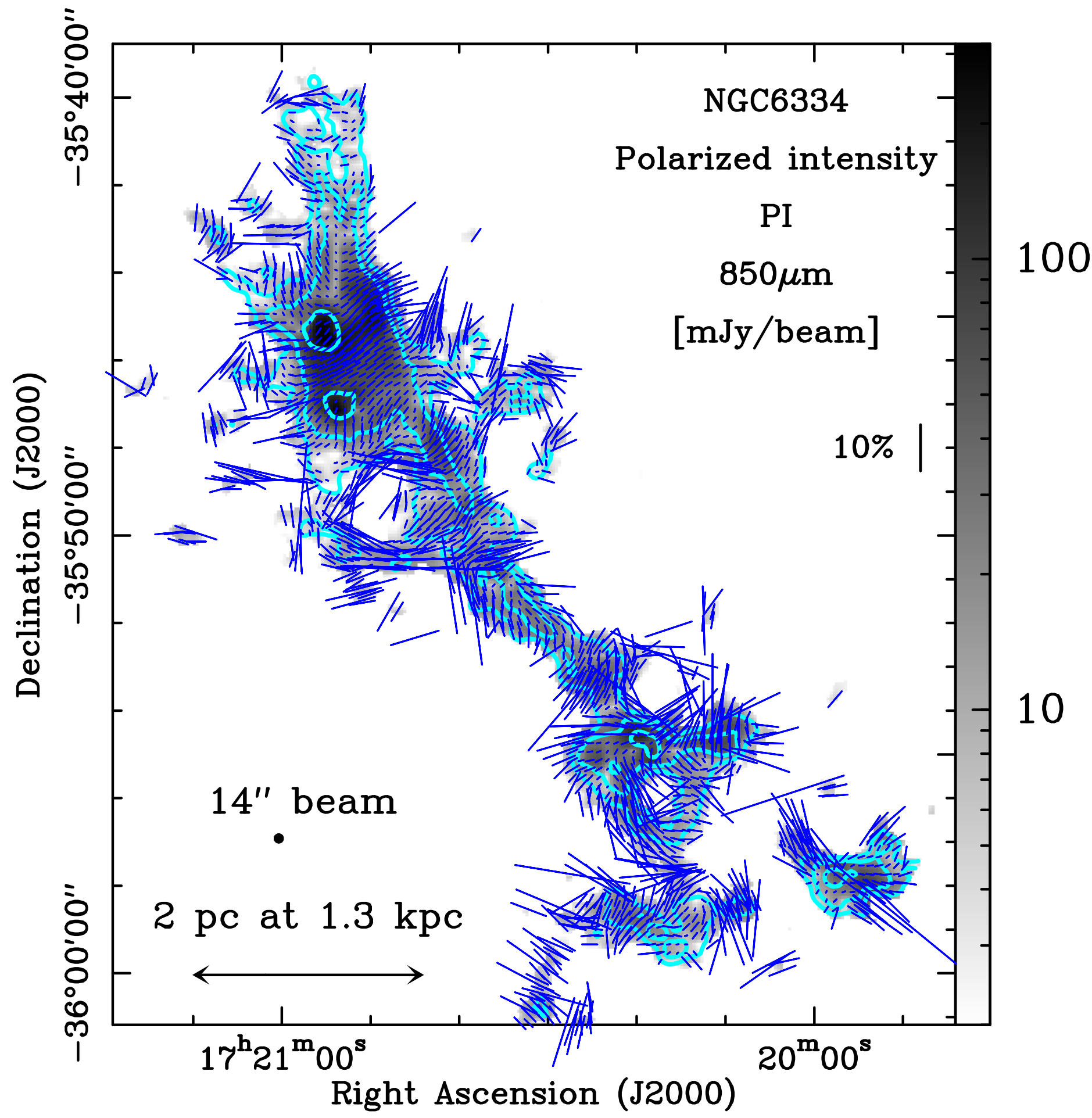

Fig. 4. Polarized intensity (PI) map (c.f., Fig. 1-top right). The blue short lines show the orientation of the POS B-field angle (c.f., Fig. 3) and their lengths are proportional to the polarization fraction. A line showing a polarization fraction of $10 \%$ is indicated on the plot. The contours are $I=0.4,1.4$, and $8 \mathrm{Jy}_{\text {beam }}{ }^{-1}$, as in Fig. 1.

To estimate the missing flux of the BISTRO data, we compare the total and polarized intensities of the BISTRO and Planck maps at the same $5^{\prime}$ resolution and towards the same area. The mean values derived from Planck are $I^{\mathrm{p}} \sim 206 \mathrm{MJy} / \mathrm{sr}$ and $P I^{\mathrm{P}} \sim 3.4 \mathrm{MJy} / \mathrm{sr}$. The mean values derived from Planck data in the surroundings of the BISTRO map are $I^{\text {p,Galactic } ~}$ $100 \mathrm{MJy} / \mathrm{sr}$ and $P I^{\mathrm{p} \text {,Galactic }} \sim 1.5 \mathrm{MJy} / \mathrm{sr}$ (corresponding to the closest red contour to the blue contour in Fig. 5). The mean values derived from BISTRO data smoothed to the $5^{\prime}$ resolu- tion are $I^{5^{\prime}} \sim 106 \mathrm{MJy} / \mathrm{sr}$ and $P I^{5^{\prime}} \sim 1 \mathrm{MJy} / \mathrm{sr}$. The $I^{5^{\prime}}$ value is equal to the "Galactic emission" subtracted Planck value of

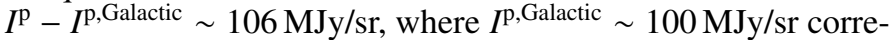
sponds to a column density value $\sim 2.4 \times 10^{22} \mathrm{~cm}^{-2}$. The $P I^{5^{\prime}}$ value is compatible within a factor of 2 with the "Galactic emission" subtracted Planck value of $P I^{\mathrm{p}}-P I^{\mathrm{p} \text {,Galactic }} \sim 1.9 \mathrm{MJy} / \mathrm{sr}$.

This comparison with the Planck data suggests that the BISTRO data recover most of the total and polarized intensity of the NGC 6334 filaments and are essentially free from large 


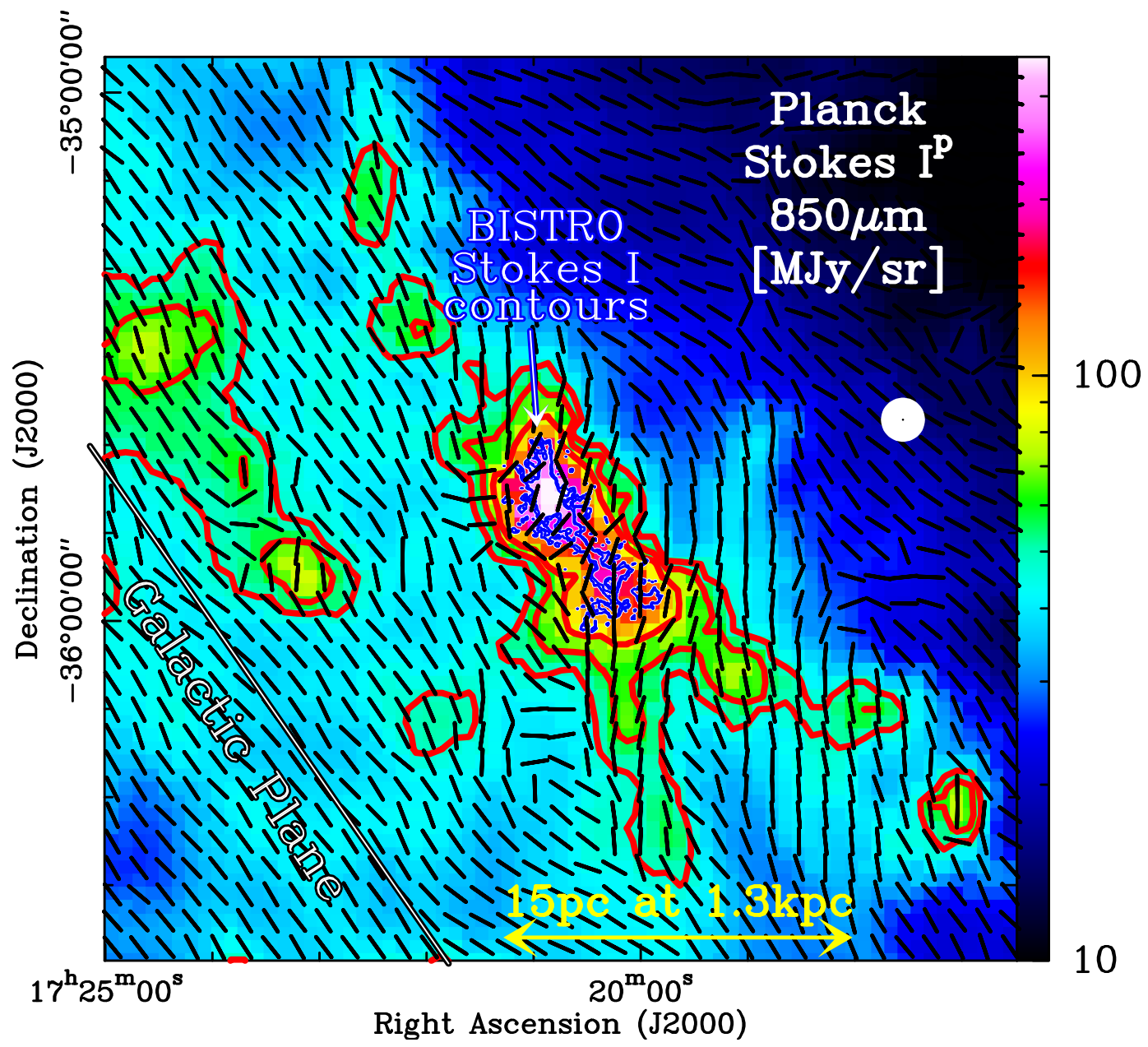

Fig. 5. Planck total intensity $I^{\mathrm{P}}$ map of the $850 \mu \mathrm{m}$ emission towards NGC 6334 . The red contours show the Planck intensity at $I^{\mathrm{P}} \sim 48,60$, and $80 \mathrm{MJy} / \mathrm{sr}$ (at 5' resolution) and the blue contour the BISTRO intensity $0.4 \mathrm{Jy} / 14^{\prime \prime}$-beam or $75 \mathrm{MJy} / \mathrm{sr}$ (at $14^{\prime \prime}$ resolution). The black lines are the POS B-field angles derived from Planck data at the resolution of $5^{\prime}$. The Planck (5') and BISTRO (14") beams are shown on the right hand side of the plot as concentric filled white and black circles, respectively. The Galactic Plane is also indicated.

scale emission corresponding to extended emission physically linked to the NGC 6334 molecular cloud in addition to lineof-sight foreground/background emission. Since, here, we are interested in studying the polarization properties of this cold and dense gas component towards NGC 6334, BISTRO highangular-resolution and high-sensitivity data are very powerful for such an analysis. If we were to analyse Planck data of NGC 6334, we would have had to remove the contribution to the emission of the surrounding ISM following the method described in, e.g., Planck Collaboration Int. XXXIII (2016).

\section{Statistical characterization of the whole field}

\subsection{Histogram and dispersion of the B-field angles}

Figure 6-top shows the histogram of the plane-of-the-sky Bfield angles $\chi_{B_{\mathrm{POS}}}$ towards the whole field for $\operatorname{SNR}(I)>25$ and $S N R(P I)>3$. The distribution of $\chi_{B_{\mathrm{POS}}}$ spans all the possible values (from $0^{\circ}$ to $180^{\circ}$ ). There is also a peak at an angle of $\sim 130^{\circ}$, which corresponds to the mean $\chi_{B_{\mathrm{POS}}}^{\mathrm{p}}$ observed by Planck (at a resolution of $5^{\prime}$ ) towards the same region (Fig. 5). The mean POS orientation of the filamentary structure of NGC 6334 elongated Northeast-Southwest is measured to be $\theta_{\text {fil }} \sim 30^{\circ}$ (East of North). Thus, while there is a large observed scatter of $\chi_{B_{\mathrm{POS}}}$ towards NGC 6334 , about $40 \%$ of the $\chi_{B_{\mathrm{POS}}}$ are within $\pm 25^{\circ}$ of being perpendicular to the filament.

All the $\chi_{B_{\mathrm{POS}}}$ measurements for $S N R(I)>25$ and $S N R(P I)>$ 3 have statistical uncertainties smaller than $10^{\circ}$ with typical uncertainties of $\sim 7^{\circ}, \sim 4^{\circ}$, and $\sim 1^{\circ}$ for $3<S N R(P I)<5$, $5<S N R(P I)<10$, and $S N R(P I)>10$, respectively (see Fig. A.2right). The observed scatter in $\chi_{B \mathrm{POS}}$ is much larger than the intrinsic uncertainties on the measurements and thus traces the variation of the B-field structure over the whole NGC 6334 region.

Figure 3 displays the spatial distribution of $\chi_{B_{\mathrm{POS}}}$ over the whole field indicating that the observed statistical scatter does not result from a completely random field. Indeed, the $\chi_{B_{\text {POS }}}$ values seem to be uniform in patches. This morphology suggests a locally ordered magnetic field with a varying $B_{\mathrm{POS}}$ structure over larger scales. To assess this "ordered variation" of the B-field, we calculate the dispersion function of $\chi_{B_{\mathrm{POS}}}$ given by

$S(x)=\sqrt{\frac{1}{N} \sum_{i=1}^{N}\left[\chi_{B_{\mathrm{POS}}}(x)-\chi_{B_{\mathrm{POS}}}\left(x_{i}\right)\right]^{2}}$,

where $x$ is the pixel position, $x_{i}$ is the neighbouring pixel to $x$, and $N$ is the number of $x_{i}$ pixels surrounding the pixel $x$. Here we are interested in the measure of the local dispersion of $\chi_{B_{\mathrm{POS}}}$. 

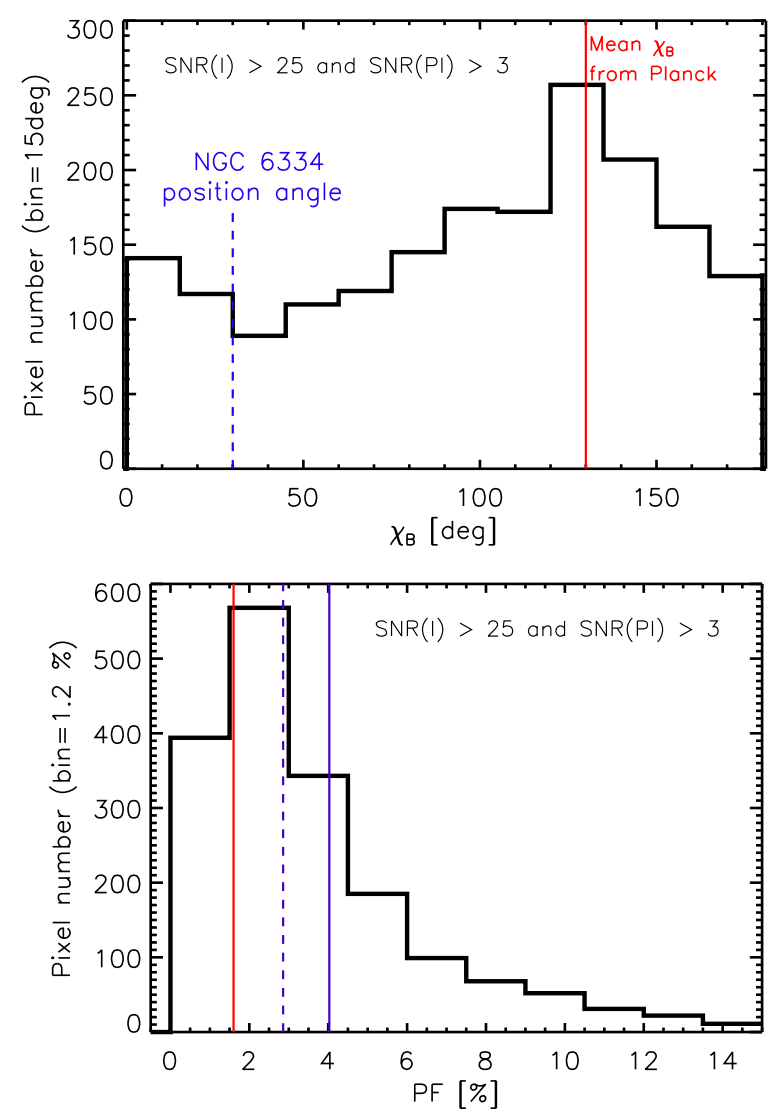

Fig. 6. Top: Distribution of $\chi_{B_{\mathrm{POS}}}$. The blue dashed line at $30^{\circ}$ indicates the mean position angle $\left(\theta_{\text {fil }}\right.$ of NGC 6334 . The red solid line at $130^{\circ}$ shows the mean $\chi_{B \mathrm{POS}}^{\mathrm{p}}$ derived from Planck data (at the resolution of $5^{\prime}$ ) towards the field observed with BISTRO (cf., Fig. 5). Bottom: Distribution of the polarization fraction $P F$. The solid and dashed blue lines indicate the mean and median values of $P F$ at $14^{\prime \prime}$, respectively. The solid red line shows the mean $P F^{\mathrm{p}}$ value derived from Planck data at $5^{\prime}$ resolution towards the BISTRO field.

We calculate $S$ for the nearest four and next-nearest four neighbours surrounding a given pixel on a regular grid, i.e., $N=8$ corresponding to a box of $3 \times 3$ pixels or $36^{\prime \prime} \times 36^{\prime \prime}$. For each pixel position, the $\left|\chi_{B_{\mathrm{POS}}}(x)-\chi_{B_{\mathrm{POS}}}\left(x_{i}\right)\right| \leq 90^{\circ}$ condition is imposed on the right hand side of Eq. 4 and the supplementary angle $\left(180^{\circ}-\left|\chi_{B_{\mathrm{POS}}}(x)-\chi_{B_{\mathrm{POS}}}\left(x_{i}\right)\right|\right)$ is taken otherwise.

The map of $S$ is shown in Fig. 7 where patches of low $\chi_{B_{\mathrm{POS}}}$ dispersions (i.e., ordered B-field structure) are separated by large values of $S$.

\subsection{Histogram of the polarization fraction}

Figure 6-bottom shows the distribution of the polarization fraction, $P F$, derived from the BISTRO data for $S N R(I)>25$ and $S N R(P I)>3$. The mean, median, and standard deviation are $\sim 4 \%, \sim 2.9 \%$, and $\sim 3.9 \%$, respectively. Typical statistical uncertainties $^{4}$ of the $P F$ measurements are $1.1 \%, 0.7 \%$, and

\footnotetext{
4 These statistical uncertainties are derived from sinusoidal function fitting to the sinusoidal time series data obtained with the POL2-DAISY observation mode of POL-2. The absolute $P F$ values discussed in this paper are corrected from the instrumental polarization (IP) offset, with a systematic uncertainty on the IP model estimated to be about $0.3 \%$ (c.f., https://www.eaobservatory.org/jcmt/2019/05/investigatingthe-effects-of-uncertainties-in-the-pol2-ip-model/) as derived
} from the BISTRO DR3 August 2019 IP model

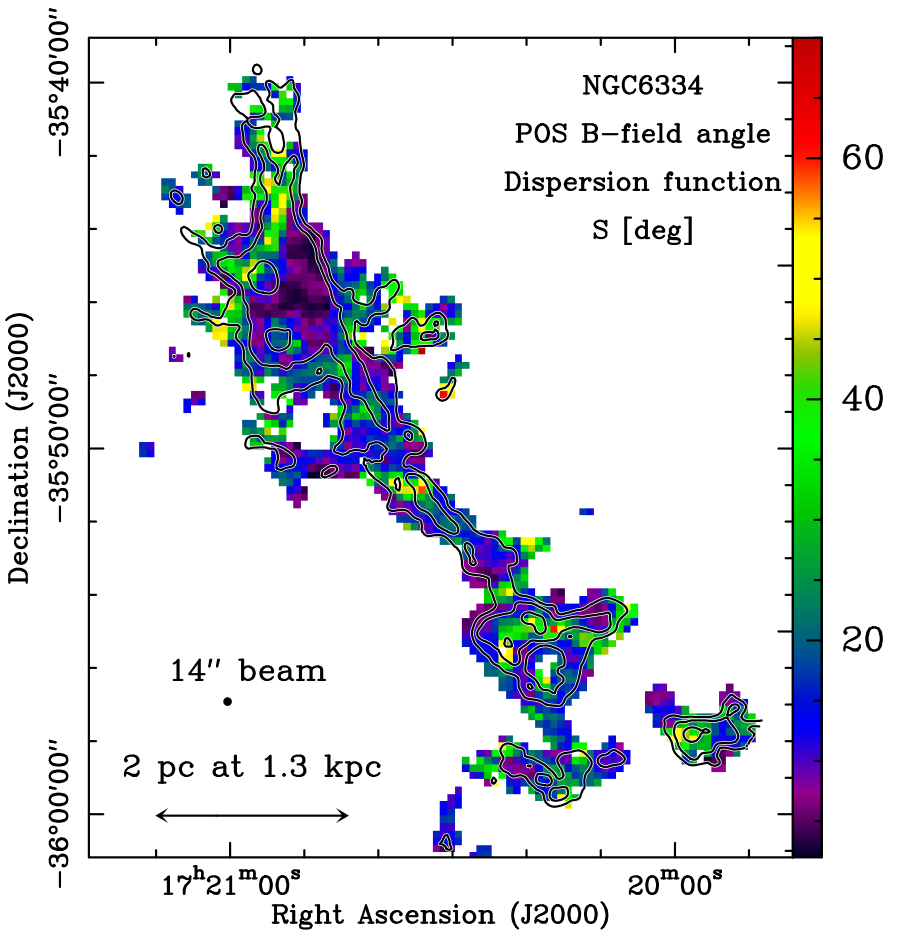

Fig. 7. Map of $S$ (dispersion function of $\chi_{B_{\mathrm{POS}}}$, see Eq. 4) for $S N R(I)>$ 25 and $S N R(P I)>3$. The contours are $I=0.4,1.4$, and $8 \mathrm{Jy} \mathrm{beam}^{-1}$, as in Fig. 1.

$0.2 \%$ for samples with $3<S N R(P I)<5,5<S N R(P I)<10$, and $S N R(P I)>10$, respectively (c.f., Fig. A.2-middle). The scatter of $P F$ observed here is larger than the measurement uncertainties and may be used to study the physical origin of the $P F$ distribution. The mean polarization fraction observed by Planck towards the same region mapped by BISTRO observations is equal to $\sim 1.6 \%$ at the $5^{\prime}$ resolution. The lower polarization fraction at the coarser Planck resolution is probably due to depolarization within the beam of unresolved B-field structure.

\subsection{Scatter plots and correlations between the parameters}

Figure 8 shows scatter plots between $I, P I$, and $P F$. We note that the two plots shown in Fig. 8 are different projections of three quantities $(I, P I$, and $P F)$, which are, by definition, not independent (with $P F=P I / I$, see Eq. 3). We thus expect clear relations among the distributions. We discuss this below.

We estimate the mean and median values of $\log (P I)$ and $\log (P F)$ as well as their $16 \%$ and $84 \%$ percentiles (corresponding to the standard deviation of a normal distribution) per bin of $\log (I)$ for data points with $S N R(I)>25$ and $S N R(P I)>3$. We also show, the distribution of the data with $1<S N R(P I)<3$ in polarized intensity (that are not correctly debiased here) as a reminder of possible shortcomings in our interpretation due to the removal of low $S N R(P I)$ and small $P F$ data points when only high-SNR $(S N R(P I)>3)$ data points are selected. For $I<150 \mathrm{mJy} /$ beam (indicated as a dotted vertical line in Fig. 8) the mean/median of the distributions of $P I$ versus $I$ and $P F$ versus $I$ are significantly affected by the selection effect of highSNR data points.

(https://www.eaobservatory.org/jcmt/2019/08/new-ip-models-forpol2-data/). 

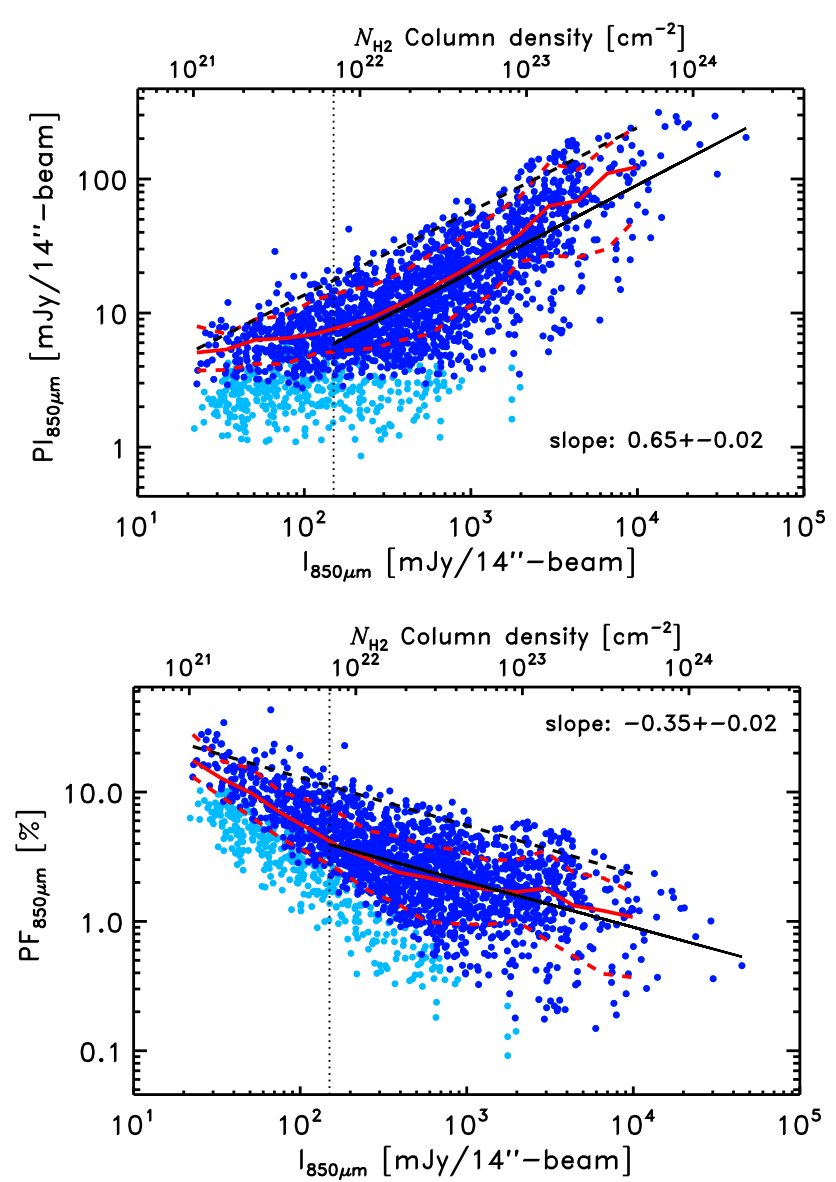

Fig. 8. Top: $P I$ versus $I$ for $S N R(I)>25$. The light and dark blue symbols are data points with $1<S N R(P I)<3$ and $S N R(P I)>3$, respectively. The dots correspond to $12^{\prime \prime}$ pixels. The red solid curve shows the median $\log (P I)$ per bin of $\log (I)$. The lower and the upper dashed red lines show the $16 \%$ and $84 \%$ percentiles, respectively. The black solid line is a linear fit to the distribution for $S N R(P I)>3$ and $I>150 \mathrm{mJy} /$ beam (indicated by the dotted vertical line). The value of the slope is given on the plot. The black dashed line is a linear fit to the upper $95 \%$ percentiles (see Sect. 3.3). The upper $x$-axis gives an estimate of the $N_{\mathrm{H}_{2}}$ column density (see Sect. 2.3). Bottom: $P F$ versus $I$. The dots, curves, and colours are the same as the upper plot for $P F$ versus $I$.

Since the polarized emission depends to first order (for an ordered B-field structure along the LOS and uniform grain alignment efficiency) on the column density of dust present along the LOS emitting polarized emission, $P I$ increases with increasing I. We can see this positive trend over $\sim 3$ orders of magnitude in both $P I$ and $I$, as $P I \propto I^{0.65 \pm 0.02}$, where the slope is derived from a linear fit (in log-log scale) to the data points for $I \geq 150 \mathrm{mJy} / \mathrm{beam}$. We can also notice, for a given value of $I$, a large scatter in $P I$. This scatter by more than one order of magnitude in $P I$ is seen for all the $I$ values from a few $10 \mathrm{mJy} / \mathrm{beam}$ to $10^{4} \mathrm{mJy} / \mathrm{beam}$, i.e., spanning $\sim 3$ orders of magnitude in $I$. With such a scatter a linear fit to the upper envelope of the distribution may indicate the maximum observed $P I$ for a given $I$. In addition, this upper envelope is more robust with respect to high-SNR data selection effects, while it may be limited by the angular resolution of the observations. A fit to the upper envelope yields a slope of $0.62 \pm 0.03$ similar to the slope derived for the full distribution for $S N R(P I)>3$ and $I \geq 150 \mathrm{mJy} / \mathrm{beam}$.

Figure 8-Bottom shows the distribution of $P F$ as a function of $I$. There is an overall decreasing trend of $P F$ with increas- ing $I$ over $\sim 2$ orders of magnitude in $P F$ (from $\sim 0.2 \%$ to $\sim 20 \%$ ) with $P F \propto I^{-0.35 \pm 0.02}$. The slope is derived from a linear fit (in log-log scale) to the data points for $S N R(P I)>3$ and $I \geq 150 \mathrm{mJy} / \mathrm{beam}$. This best fit slope is expected from the result of Fig. 8-Top since $P F=P I / I$ and $P I \propto I^{0.65 \pm 0.02}$. A steeper slope $\left(P F \propto I^{-0.46 \pm 0.01}\right)$ is obtained if the full distribution (for $S N R(P I)>3$ ) is fitted. A fit to the upper envelope (not affected by the selection criteria of high-SNR) yields a slope of $-0.37 \pm 0.03$ similar to the slope derived for the full distribution for $S N R(P I)>3$ and $I \geq 150 \mathrm{mJy} / \mathrm{beam}$.

The scatter plot of $P F$ versus $I$ indicates a large scatter in $P F$ (of $\sim 2$ orders of magnitude) for a given value of $I$. Such a scatter was shown earlier by the statistical analysis of the Planck polarization data on large scales (Planck Collaboration Int. XIX 2015; Planck Collaboration Int. XLIV 2016) as well as at smaller scales in other regions analysed within the BISTRO survey (Kwon et al. 2018; Soam et al. 2018; Wang et al. 2019). We find here, however, slopes significantly shallower than those shown before, e.g., Soam et al. (2018) and Wang et al. (2019) reported slopes of -0.9 and -1 , respectively (see also similar results from the analysis of SMA and ALMA data, e.g., Chen et al. 2012; Koch et al. 2018).
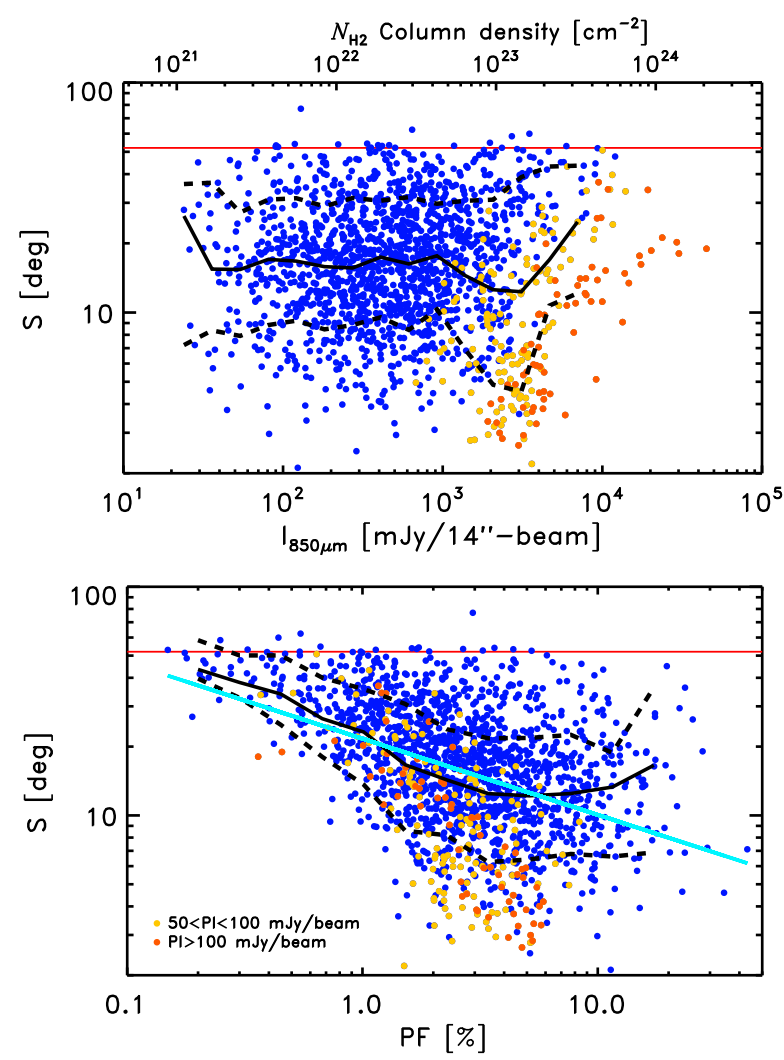

Fig. 9. Scatter plot of $S$ (dispersion function of $\chi_{B \mathrm{POS}}$ ) as a function of $I$ (top) and $P F($ bottom) for data points with $S N R(I)>25$ and $S N R(P I)>$ 3 (c.f., Fig. 7). The data points with $50<P I<100 \mathrm{mJy} / \mathrm{beam}$ and $P I>100 \mathrm{mJy} / \mathrm{beam}$ are shown in yellow and red, respectively. The black solid curves show the median $\log (S)$ per bin of $\log (I)$ and $\log (P F)$ in the top and bottom panels, respectively. The lower and the upper dashed black lines are the $16 \%$ and $84 \%$ percentiles of the distributions, respectively. The red horizontal line at $S=52^{\circ}$ shows the value that $S$ would take for a B-field dominated by turbulence. In the bottom panel, the cyan solid line is a linear fit to the distribution.

Figure 9 shows the distribution of $S$ (dispersion function of $\chi_{B_{\text {POS }}}$, see Eq. 4 and Fig. 7) as a function of $I$ or $P F$ for the whole field. While there is no particular trend seen between $S$ and $I$, an 
anti-correlation between $S$ and $P F$ can be noticed (with a slope of -0.34). We note, however, that this latter anti-correlation may be the result of the selection of data points with $S N R(P I)>3$ removing those measurements with $P F \lesssim 1 \%$. A large spread of the values of $S$ for a given value of $P F$ can be seen, especially for $P F>1 \%$. Such statistical distribution of $S$ as a function of $P F$ is also seen with Planck towards the whole sky (e.g., Planck Collaboration Int. XIX 2015) as well as on much smaller scales with ALMA towards high-mass star forming regions (e.g., Koch et al. 2018).

We discuss possible origins of these scatters of the observed polarization properties in Sects. 6.1 and 6.3 below.

\section{Properties of the different crests identified towards the NGC 6334 filament network}

The molecular cloud structure of NGC 6334, on large scales, may be described as a "ridge-dominated" structure (cf. Sect. 1) with a main $\sim 10 \mathrm{pc}$-long ridge threaded by a network of shorter filamentary structures, the "sub-filaments", connected to the ridge from the side. In the North of the field, two dense and compact star-forming hubs, I and $\mathrm{I}(\mathrm{N})$, can be seen located at the junction of multiple sub-filaments as presented by Sandell (2000) and observed recently at high-resolution with ALMA (Sadaghiani et al. 2020). These two sources are in turn observed towards a larger ellipsoidal $\sim 1 \mathrm{pc} \times 2 \mathrm{pc}$-scale hub-like structure (see Fig. 10) encompassing also a section of the ridge, and the inner-parts of the sub-filaments. To distinguish between these hub structures observed at different scales, hereafter we refer to the $\sim 0.1 \mathrm{pc}$-scale I and $\mathrm{I}(\mathrm{N})$ sources as core-hubs and the $\sim 1 \mathrm{pc}$ scale structure as a clump-hub (cf., Fig. 10).

We use the DisPer $\mathrm{SE}^{5}$ algorithm to trace the elongated dusty crests seen on the $850 \mu \mathrm{m}$ Stokes $I$ map at a resolution of $14^{\prime \prime}$ (projected onto a grid with $4^{\prime \prime}$ pixel size). The crests traced by DisPerSE may correspond to "filament-crests" or to "shellcrests" associated with shell-like structures around H II regions (such as those around sources II and IV). We mention briefly possible differences between "filament-crests" and "shell-crests" below (see also Zavagno et al. 2020, who present a study of the properties of shell-crests and filament-crests in the RCW 120 $\mathrm{H}$ II region).

In the following, we study the physical properties of the 14 crests identified towards the NGC 6334 filament network (Fig. 10). We discuss the observed values along the ridge (crests 1 to 4$)^{6}$ and the variation of the properties at both ends of the 10 sub-filaments (crests 5 to 14) from their outer parts to their inner parts connected to the clump-hub. We include in this analysis crests 10 and 11, which have one of their ends connected to the clump-hub or the ridge, even though these two crests may be better described as shell-like structures associated with the H II region of source II. We discuss this further below. Table 1 summarizes the mean properties and their respective dispersions for the 14 filament crests. Table 2 provides estimated quantities that describe the stability of these filaments.

\footnotetext{
5 See Sousbie (2011) and Sousbie et al. (2011) for a description of the method and Arzoumanian et al. (2011) for the first application of this algorithm in the context of tracing the crests of interstellar filaments.

${ }^{6}$ For the purpose of this study, which is aimed at describing the variation of the properties along the NGC 6334 elongated dusty structure, we do not include other crests traced by DisPerSE around source IV.
}

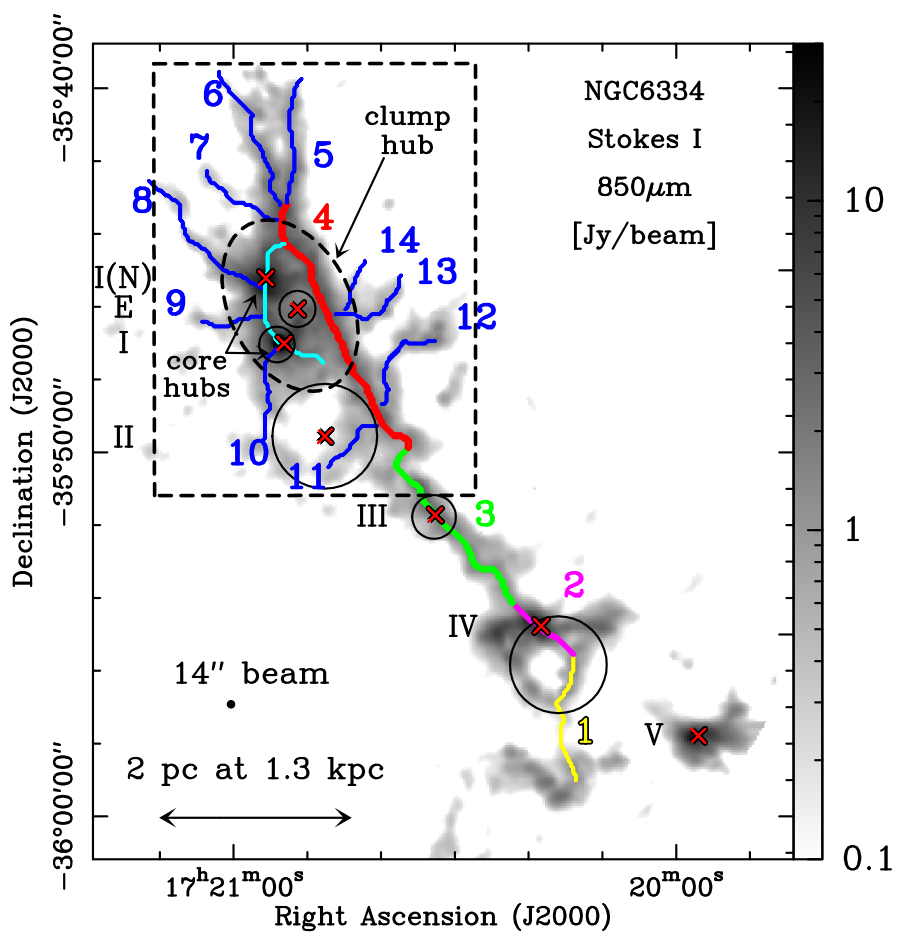

Fig. 10. The crests of the identified filamentary structures are overplotted on the Stokes $I$ map. The connected sections from 1 to 4 trace the ridge crest (cf., Figs. 11 and 12). The blue lines from 5 to 14 trace the crests of the sub-filaments. The cyan line traces the crest of a filament connecting the two core-hubs, I and I(N). The black dashed rectangle indicates the hub-filament structure (see Fig. 13). The black dashed ellipse shows the clump-hub. The crosses and circles are the same as in Fig. 1-top-left.

\subsection{Physical parameters along the ridge crest}

\subsubsection{Polarization properties}

Figure 11 shows the variation of $I, P I$, and $P F$ along the ridge crest from the South to the North. For comparison, we also plot the column density estimated from SPIRE+ArTéMiS data at $350 \mu \mathrm{m}$ (André et al. 2016) after subtracting the "Galactic emission" corresponding to the extended emission filtered out in the BISTRO data (cf. Sect. 2.3). We can see that both data trace essentially the same dust continuum emission.

We notice two clear peaks in $P I$ at $z \sim 1.8 \mathrm{pc}$ and $z \sim 5.8 \mathrm{pc}$. The former is associated to a peak in $I$ (with a maximum value of $12.6 \mathrm{Jy} / \mathrm{beam}$ ) and a trough in $P F$. This position corresponds to the active star-formation site IV (see Fig. 10). The latter PI peak, however, is not associated with a strong peak in $I$ nor with a decrease in $P F$ as usually expected (c.f., Sect. 3.3). Indeed, the distribution of $P F$ along the ridge crest has a complex structure, with several relatively compact zones (roughly $0.2-0.4 \mathrm{pc}$ ) with moderately high values $\left(P F>3 \%\right.$ and $\left.N_{\mathrm{H}_{2}} \sim 10^{23} \mathrm{~cm}^{-2}\right)$, and these localised regions of $P F$ are neither correlated nor anticorrelated with $I$. Between $z \sim 2.2 \mathrm{pc}$ and $z \sim 3.2 \mathrm{pc}, I$ increases by a factor $\sim 3$, while $P I$ and $P F$ decrease by a factor of $\sim 2$ and $\sim 6$, respectively. On the other hand, between $z \sim 5 \mathrm{pc}$ and $z \sim 5.8 \mathrm{pc}$, there is an overall increase in the three quantities $I$, $P I$, and $P F$ by factors of $\sim 2, \sim 13$, and $\sim 7$, respectively. We discuss physical possibilities for these variations in Sect. 6.3.

On average, the position angle of the ridge is $\theta_{\text {fil }} \sim 30^{\circ}$ and the prevailing $B_{\text {pos }}$ field orientation (dominated by $\chi_{B_{\mathrm{POS}}}$ of the crest 4) is close to perpendicular to the elongated ridge (cf., Table 1), as already suggested by Planck observations (see 

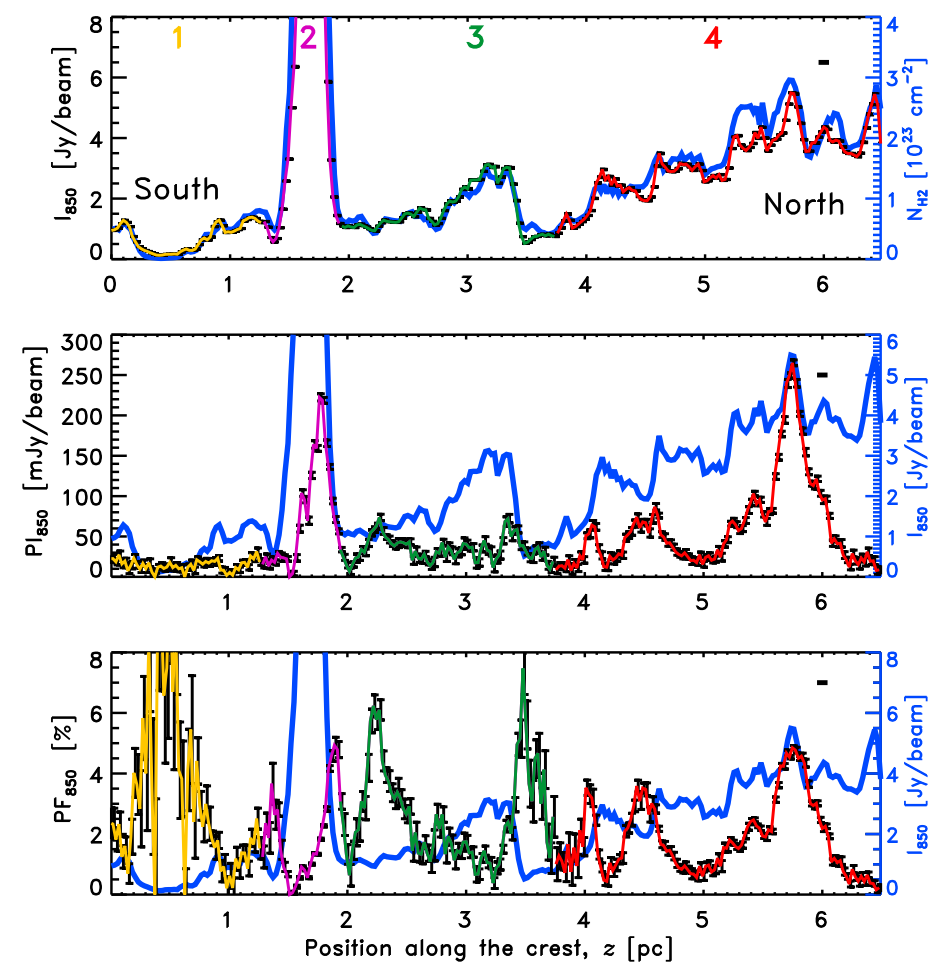

Fig. 11. Observed $I$ (top), $P I$ (middle), and $P F$ (bottom) along the ridge crest for the sections from 1 to 4 (indicated with different colours, see Fig. 10) from South to North (left to right on the horizontal axes). The short vertical black bars show the $\pm \sigma$ statistical uncertainties. The horizontal black segments on the top right hand of the panels indicate the $14^{\prime \prime}(0.09 \mathrm{pc})$ beam size of the data. The blue curve on the top panel is the column density derived from SPIRE+ArTéMiS data after subtraction of a constant "Galactic emission" value of $3 \times 10^{22} \mathrm{~cm}^{-2}$ (cf. Sect. 2.3). The blue curves on the middle and bottom panels show $I$ along the ridge crest (same as the coloured curve on the top panel).

Fig. 5). We notice, however, some sections along the ridge crest where the relative orientation between the B-field and the ridge changes. These variations are shown in Fig. 12 where we plot $\chi_{B_{\text {POS }}}, \theta_{\text {fil }}$ (the filament orientation), and $\phi_{\text {diff }}=\left|\theta_{\text {fil }}-\chi_{B_{\text {pos }}}\right|$ along crests 3 and 4 . The orientation of the crest, $\theta_{\text {fil }}$, is measured on the Stokes $I$ map using the Hessian matrix (c.f., Eq. 3 in Arzoumanian et al. 2019). Along the $\sim 3$ pc crest $4, \phi_{\text {diff }}$ is mostly uniform and close to $90^{\circ}$, except for some localised regions (between $z \sim 3.8-4.0 \mathrm{pc}$ and $z \sim 4.6-4.9 \mathrm{pc}$ ) where $\phi_{\text {diff }}$ fluctuates between $30^{\circ}$ and $60^{\circ}$, i.e., neither perpendicular nor parallel orientation. Along the $\sim 2 \mathrm{pc}$ crest $3, \phi_{\text {diff }}$ oscillates between mostly perpendicular to mostly random to mostly parallel from South to North (see Sect. 6.2 for a discussion).

\subsubsection{Magnetic field strength and stability parameters}

Dust polarized thermal emission observations do not provide a direct measurement of the B-field strength, which is critical to study the stability and dynamical evolution of filaments and the role of the B-field in the star formation process. Indirect methods, such as the Davis-Chandrasekhar-Fermi (DCF) method (Davis \& Greenstein 1951; Chandrasekhar \& Fermi 1953) calibrated on magnetohydrodynamic (MHD) simulations (e.g., Ostriker et al. 2001; Heitsch et al. 2001; Falceta-Gonçalves et al. 2008), can provide an estimate of the POS B-field strength.

We here use the DCF method to estimate the $B_{\mathrm{POS}}$ field strength, combining estimates of the volume density $n_{\mathrm{H}_{2}}$ (in
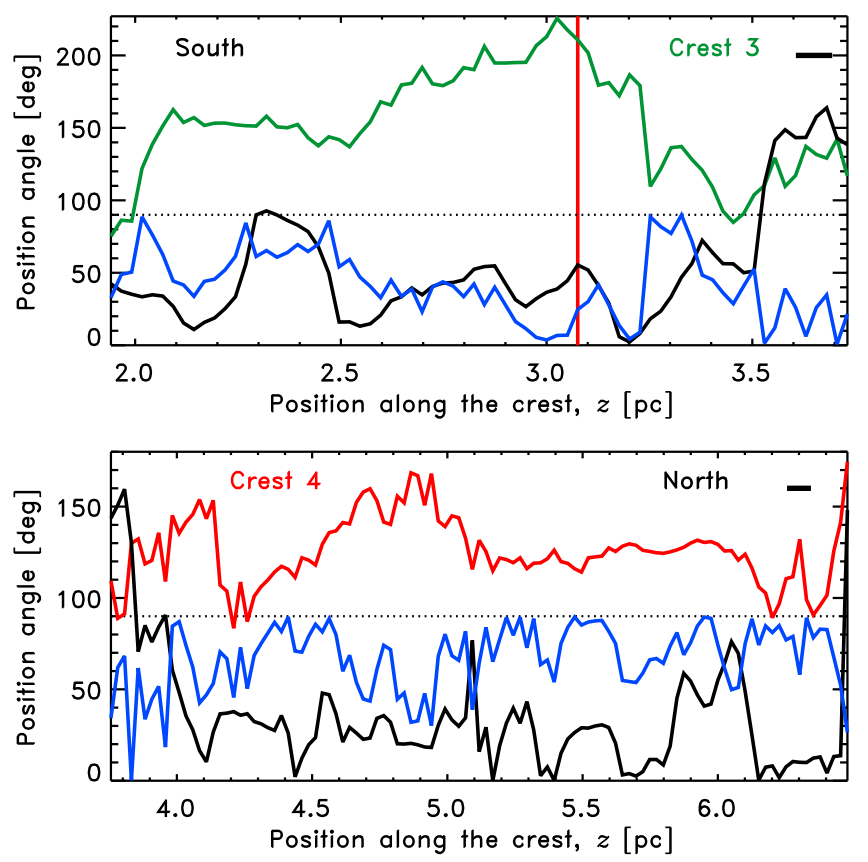

Fig. 12. Variation of the position angles of $\chi_{B_{\mathrm{POS}}}$ (green in the top and red in the bottom), $\theta_{\text {fil }}$ (black), and $\phi_{\text {diff }}=\left|\theta_{\text {fil }}-\chi_{B_{\text {pos }}}\right|$ (blue) along crests 3 (top) and 4 (bottom), from South to North. Only pixels along the crests are considered. The dotted horizontal lines show an angle of $90^{\circ}$. The vertical red line in the top panel is the position of source III (cf., Fig. 10). The horizontal black segments on the top right hand of the panels indicate the $14^{\prime \prime}(0.09 \mathrm{pc})$ beam size of the data.

$\mathrm{cm}^{-3}$ ), the non-thermal velocity dispersion $\sigma_{\mathrm{NT}}$ (in $\mathrm{km} / \mathrm{s}$ ), and the POS B-field angle dispersion $\sigma_{\chi_{B \text { pos }}}$ (in degrees), using the following relation (cf., e.g., Crutcher et al. 2004):

$B_{\mathrm{POS}}=9.3 \sqrt{n_{\mathrm{H}_{2}}} \frac{\sigma_{\mathrm{NT}} \sqrt{8 \ln 2}}{\sigma_{\chi_{\mathrm{B} \text { pS }}}}$

in units of $\mu \mathrm{G}$. We take $n_{\mathrm{H}_{2}}=N_{\mathrm{H}_{2}} / W_{\text {fil }}$ from the $N_{\mathrm{H}_{2}}$ values (derived as explained in Sect. 2.3 for $T=20 \pm 5 \mathrm{~K}$ ) and a filament width of $W_{\text {fil }}=0.11 \pm 0.04$ pc (André et al. 2016). The $\sigma_{\chi_{B \mathrm{pOS}}}$ values correspond to the median absolute deviation, $\mathrm{mad}$, of the $\chi_{B_{\text {pos }}}$ measurements observed at each pixel position along a given crest, i.e., $\sigma_{\chi_{\text {BPOS }}}=\operatorname{median}\left[\left|\chi_{B_{\mathrm{POS}}}-\operatorname{median}\left(\chi_{B_{\mathrm{pos}}}\right)\right|\right]$. These values are given in Table 1 (column 8). Here we compute the mad value, which is a more robust measure of the dispersion than the standard deviation, which is more affected by outliers in non normal distributions. We also note that the $\sigma_{\chi_{B \mathrm{POS}}}$ values calculated here are upper limits since possible large-scale variations of the B-field structure (which have not been removed) may increase the measured dispersions. We refer to a future work for detailed modelling of the B-field structure along and across the filaments. This work will be done in combination with the velocity structure derived from molecular line observations of similar resolution as the BISTRO data.

There are no available spectroscopic observations tracing the velocity structure of the dense gas towards the $10 \mathrm{pc}-$ long NGC 6334 filamentary structure at comparable resolution of the BISTRO data ${ }^{7}$. To estimate $\sigma_{v}$, we use the $\sigma_{v} \sim N_{\mathrm{H}_{2}}^{0.35}$ relation

7 The $\mathrm{N}_{2} \mathrm{H}^{+}(1-0)$ spectra observed along the NGC 6334 ridge as part of the MALT90 survey (Jackson et al. 2013) with the MOPRA 22-m telescope at the angular resolution of $40^{\prime \prime}$ show $\sigma_{v} \gtrsim 1 \mathrm{~km} \mathrm{~s}^{-1}$. This latter velocity dispersion is much larger than the values observed towards 
suggested by Arzoumanian et al. (2013) from the analysis of $\mathrm{N}_{2} \mathrm{H}^{+}(1-0)$ observations (with the IRAM-30m telescope) towards a sample of supercritical filaments with line masses between $20 \mathrm{M}_{\odot} / \mathrm{pc}$ and $500 \mathrm{M}_{\odot} / \mathrm{pc}$, corresponding to a similar line mass range for the filaments studied in this paper. The derived $\sigma_{v}{ }^{8}$ values are given in column 5 of Table 2 and range between $\sim 0.4-0.6 \mathrm{~km} \mathrm{~s}^{-1}$ compatible with observational results towards other filaments of similar line masses. For example, observations towards the SDC13 filament system with the IRAM-30m at $\sim 30^{\prime \prime}$ (using $\mathrm{N}_{2} \mathrm{H}^{+}$, Peretto et al. 2014), towards dense filaments in a sample of Galactic giant molecular clouds with the GBT as part of the KEYSTONE project at 30" (using $\mathrm{NH}_{3}$, Keown et al. 2019), and towards the infrared dark cloud G14.225-0.506 with ALMA (using $\mathrm{N}_{2} \mathrm{H}^{+}$, Chen et al. 2019), all suggest values of $\sigma_{v} \sim 0.4-0.8 \mathrm{~km} \mathrm{~s}^{-1}$. In addition, the $\sigma_{v}=0.62 \mathrm{~km} \mathrm{~s}^{-1} \mathrm{de}-$ rived here for the crest 4 is similar to that derived from $\mathrm{N}_{2} \mathrm{H}^{+}$ ALMA data at $3^{\prime \prime}$ of the same region (Shimajiri et al. 2019). We calculate the non-thermal component $\sigma_{\mathrm{NT}}=\sqrt{\sigma_{v}^{2}-c_{\mathrm{s}}^{2}}$ for a temperature $T=20 \mathrm{~K}$ for NGC 6334. The uncertainty on $\sigma_{v}$ is inferred from the dispersion of $N_{\mathrm{H}_{2}}$ along each crest. The uncertainty on $\sigma_{\mathrm{NT}}$ is derived from the propagation of those on $\sigma_{v}$ and $c_{\mathrm{s}}$ (estimated for a temperature uncertainty of $5 \mathrm{~K}$ ).

The values of $B_{\mathrm{POS}}$ along the ridge crest span a range from $\sim 100 \mu \mathrm{G}$ to $\sim 800 \mu \mathrm{G}$ with an average value of $\sim 270 \pm 100 \mu \mathrm{G}$. The uncertainty on $B_{\mathrm{POS}}$ is estimated from error propagation (see the uncertainties of the different parameters in Tables 1 and 2).

To estimate the support of the B-field against gravitational collapse, we calculate the magnetic critical mass per unit length (assuming the 3D B-field structure to be close to the POS) using the relation derived by Tomisaka (2014):

$M_{\text {line,crit }}^{B}=4.48\left(W_{\text {fil }} / 0.1 \mathrm{pc}\right)\left(B_{\mathrm{POS}} / 10 \mu \mathrm{G}\right)$.

We compare this latter value with the mass per unit length $\left(M_{\text {line }}\right)$ estimating the magnetic virial parameter $\alpha_{\text {vir }}^{B}=M_{\text {line,crit }}^{B} / M_{\text {line }}$, where $M_{\text {line }}=\mu_{\mathrm{H}_{2}} m_{\mathrm{H}} N_{\mathrm{H}_{2}} W_{\text {fil }}$, with $W_{\text {fil }}=0.11 \mathrm{pc}$ is the filament width $\left(\mu_{\mathrm{H}_{2}}\right.$ and $m_{\mathrm{H}}$ are the same as in Sect.2.3). Thus $\alpha_{\mathrm{vir}}^{B} \propto$ $B_{\mathrm{POS}} / N_{\mathrm{H}_{2}} \propto\left(\sigma_{\mathrm{NT}} / \sigma_{\chi_{B_{\mathrm{pos}}}}\right) / \sqrt{N_{\mathrm{H}_{2}} W_{\text {fil }}}$.

We also derive the kinetic (thermal + turbulent) virial parameter $\alpha_{\text {vir }}^{v}=M_{\text {line,vir }}^{v} / M_{\text {line }}$ where $M_{\text {line,vir }}^{v}=2 \sigma_{v}^{2} / G$ (c.f., e.g., Fiege \& Pudritz 2000) and the effective virial parameter that takes into account the thermal, turbulent, and magnetic support, $\alpha_{\text {vir }}^{v, B}=M_{\text {line,vir }}^{v, B} / M_{\text {line }}$, where $M_{\text {line,vir }}^{v, B}=M_{\text {line,vir }}^{v}+M_{\text {line,crit }}^{B}$ (c.f., Tomisaka 2014).

The results (see Table 2) indicate that while the different sections of the ridge are highly thermally supercritical with $\alpha_{\text {cyl }}^{T}=M_{\text {line,crit }}^{T} / M_{\text {line }} \ll 1$ (with $M_{\text {line,crit }}^{T=20 \mathrm{~K}} \sim 27 \mathrm{M}_{\odot} / \mathrm{pc}$ ), magnetically supercritical with $\alpha_{\text {vir }}^{B}<1$ and gravitationally unstable with respect to the kinetic support $\alpha_{\mathrm{vir}}^{v}<1$, the combination of the magnetic and kinetic (thermal and turbulent) energies may provide effective support to the ridge as a whole against gravitational collapse with $\alpha_{\text {vir }}^{v, B} \sim 1.1 \pm 0.6$. We notice, however, some differences: for crest $3, \alpha_{\text {vir }}^{B} \sim 0.5 \pm 0.3$ suggesting that the gravitational energy dominates over the magnetic energy, which may be compatible with the change of $\chi_{B_{\mathrm{pos}}}$ along this section of the ridge (cf., Fig. 12), while for crest $4, \alpha_{\text {vir }}^{B}=1.1 \pm 0.7$ suggesting a more important internal magnetic support against gravitational collapse (see discussion Sect. 6.2).

other dense filaments at better angular resolutions (see examples in the text)

${ }^{8}$ Since the $\sigma \sim N_{\mathrm{H}_{2}}^{0.35}$ relation in Arzoumanian et al. (2013) is calibrated for a gas temperature of $T=10 \mathrm{~K}$, we rescale it here to the NGC 6334 temperature of $T=20 \mathrm{~K}$.

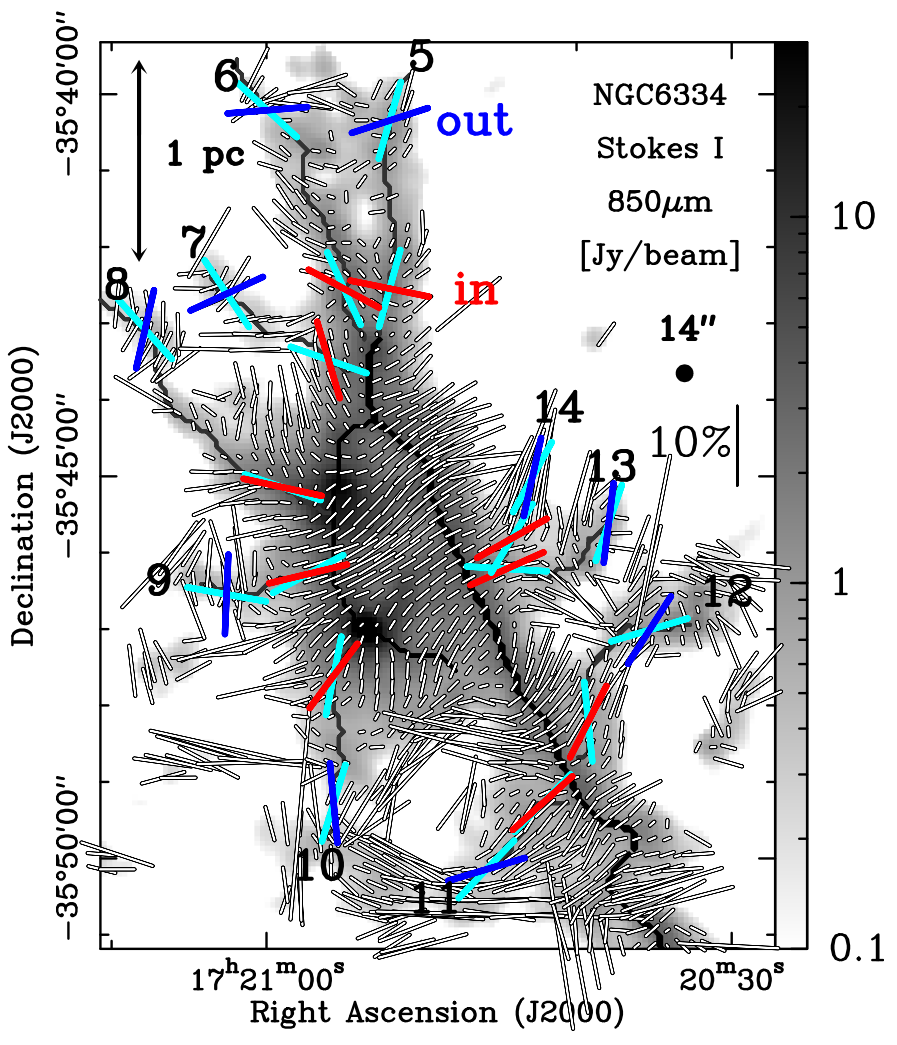

Fig. 13. Close-up of the northern region of NGC 6334 within the black dashed rectangle indicated in Fig. 10. The crests are those shown in Fig. 10 and over-plotted on the Stokes $I$ map. The white lines correspond to $\chi_{B_{\mathrm{POS}}}$ and their lengths are proportional to $P F$. On the right hand side, a vertical line shows the $10 \%$ polarization fraction scale. The black filled circle corresponds to the $14^{\prime \prime}$ beam. The blue and red lines indicate the mean values for $\chi_{B_{\mathrm{POS}}}$ for the out and in parts of the subfilaments, respectively. The cyan lines show the orientation $\left(\theta_{\text {fil }}\right)$ of the out and in parts of the sub-filaments (see Sect. 4.2, Fig. 14, and Table 1). The length of the blue, red, and cyan lines is equal to $60^{\prime \prime}$, corresponding to the extent of the circular area used to estimate the parameters.

\subsection{Physical parameters along the sub-filaments}

Figure 10 shows the crests tracing the network of the subfilaments connected to the clump-hub encompassing a section of the ridge (crest 4 ) and the two core-hubs I and $\mathrm{I}(\mathrm{N})$ that also lie along a filament traced with the cyan line in Fig. 10.

We estimate the physical properties of the sub-filaments as explained in Sect. 4.1 for the ridge. To investigate possible variations of these properties along the sub-filaments from their outerparts to their inner-parts connected to the clump-hub we give, in Tables 1 and 2, two values of the properties for each sub-filament towards their inner and outer $\sim 60^{\prime \prime}$ section of the crests, respectively. The inner-parts (in) correspond to the $33 \%$ (one-third) section of the crests connected to the clump-hub. The outer-parts (out) are the 33\% sections of the other end of the crest away from the clump-hub (cf., Fig. 13). In practice, the Stokes $I$ and the filament orientation $\theta_{\text {fil }}$ values are measured along the inner and outer $\sim 60^{\prime \prime}$ of the crests ( 4 independent $14^{\prime \prime}$ beams). The PI, $P F$, and $\chi_{B_{\text {POS }}}$ values are estimated in a circular area with a diameter of $60^{\prime \prime}$ towards the in and out parts, which yield between 10 and 14 independent measurements for a 14" beam (except for 6 out and 14 out where the values are measured towards 7 independent data points).

Figures 14 and 15 show the distribution of the properties measured towards the in and out parts of the sub-filaments. 

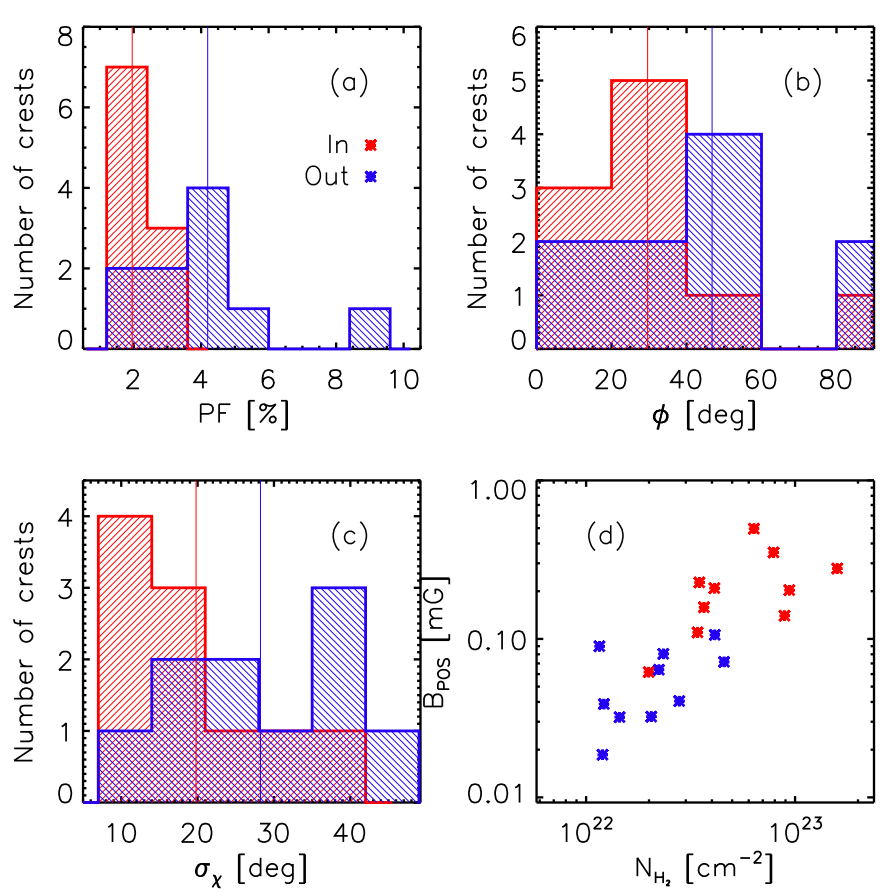

Fig. 14. Distributions of properties measured towards the in (red) and out (blue) parts of the 10 sub-filaments: a) polarization fraction, b) $\phi_{\text {diff }}=\left|\theta_{\text {fil }}-\chi_{B_{\text {pos }}}\right|$, difference between the filament orientation $\theta_{\text {fil }}$ and the POS B-field angle $\chi_{B_{\mathrm{pos}}}$, c) dispersion of $\chi_{B_{\mathrm{pos}}}$, d) POS B-field strength as a function of the column density. The red and blue vertical lines indicate the median of the distributions of the properties measured towards the in and out parts of the sub-filaments, respectively (see text for details and Tables 1 and 2 for the values).

These histograms illustrate the variation of the properties along the sub-filaments as they merge with the clump-hub. The total intensity $I$ and accordingly $N_{\mathrm{H}_{2}}$ and $M_{\text {line }}$ increase on average by factors of $\sim 2.8$ from the outer parts to the inner parts. The increase of $N_{\mathrm{H}_{2}}$ (calculated here for $T=20 \mathrm{~K}$ ) is larger than what would be expected from increases of temperature from $\sim 15 \mathrm{~K}$ to $\sim 25 \mathrm{~K}$ from the outer to the inner parts. The polarized intensity $P I$ increases on average by a factor of $\sim 1.5$ and the polarization fraction $P F$ decreases on average by a factor of $\sim 2$ (corresponding to a decrease of $\sim 2.2 \%$ on average) from the outer-parts to the inner-parts. There is, however, a larger dispersion of the $P F$ distribution in the outer part, with $\sigma_{P F}^{\text {out }} \sim 2 \%$, while in the inner part $\sigma_{P F}^{i n} \sim 0.6 \%$. The dispersion of the POS B-field angle is smaller by a factor $\sim 1.4$ on average in the inner-parts compared to the outer-parts. In the outer-parts, however, the distribution of $\sigma_{\chi_{B \mathrm{POS}}}$ spans a wider range of values. In the inner-part of the sub-filaments, the POS B-field seems to be mostly parallel to their crests, except for crest 5 (see Fig. 13). In the outer-parts, the histogram of $\phi_{\text {diff }}^{\text {out }}$ (Fig. 14b) does not show a clear trend for a preferred orientation. Towards the crests 7 and 9 the POS B-field is mostly perpendicular to the sub-filaments with relative angles $\phi_{\text {diff }} \sim 80^{\circ}$. Towards the crests 5,6 , and 8 the POS B-field has no preferred relative orientation, with $\phi_{\text {diff }}$ between $46^{\circ}$ and $55^{\circ}$. Towards the crests 10 to 14 the POS B-field is mostly parallel to the crests, with $\phi_{\text {diff }}$ between $12^{\circ}$ and $40^{\circ}$. Crests 10 and 11 are observed towards the edge of an $\mathrm{H}$ II region (i.e., Source II in Fig. 10) and may be affected by the interaction with the expanding bubble (see discussion in Sect. 6.3). Crest 14 is very short and the outer and inner parts, as we defined them here, are not really distinguishable. These variations of the relative orientation between the filament and the B-field orientations may result from the coupled dynamical evolution of the filaments and the B-fields. However, they may also be due to projection effects: A configuration that is perpendicular in projection, i.e., derived from the observations, has a high probability to be perpendicular in 3D, while a configuration that is parallel in projection may correspond to a perpendicular configuration in 3D (see Fig. C.5. in Planck Collaboration Int. XXXV 2016, and Doi et al. 2020). We discuss possible origins of the variation of the B-field orientation along the sub-filaments in Sect. 6.2.
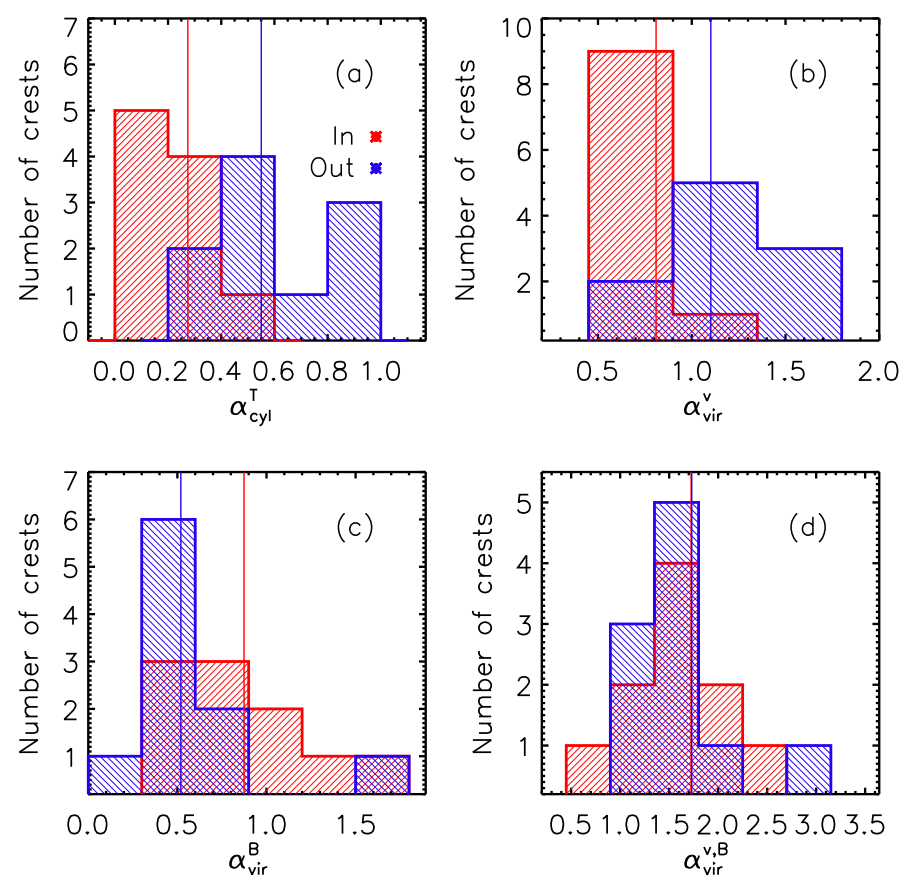

Fig. 15. Similar to Fig. 14 for the parameters describing the stability of the in (red) and out (blue) sub-filament parts: a) Thermal critical stability parameter, b) kinetic (thermal + turbulent) virial parameter, c) magnetic virial parameter, d) effective (thermal + turbulent + magnetic) virial parameter.

The sub-filaments are thermally supercritical with $\alpha_{\text {cyl }}^{T}<1$, and show an decrease of $\alpha_{\text {cyl }}^{T}$ (by a factor of $\sim 2$ on average) from out to in. The kinetic virial parameter $\alpha_{\text {vir }}^{v}$ is on average smaller in the inner parts compared to the outer parts (by a factor of 1.4 on average), while the magnetic virial parameter $\alpha_{\text {vir }}^{B}$ increases (by a factor of $\sim 1.7$ on average) from out to in. In the outer parts, $\alpha_{\mathrm{vir}}^{B} \sim 0.5 \pm 0.3<1$ suggesting that magnetic tension alone is not enough to balance gravity while the inner parts of the subfilaments seem to be in a magnetic critical balance with $\alpha_{\text {vir }}^{B} \sim$ 1. When both the magnetic and kinetic (thermal and turbulent) supports are combined, the estimated $\alpha_{\text {vir }}^{v, B} \gtrsim 1$ values suggest that the inner-parts and the outer-parts are in an overall balance between the effective pressure forces and gravity (see Sect. 6.2 for a discussion on these results).

\section{Power spectrum of intensity and B-field angle along the ridge crest}

Here we present an analysis of the one-dimensional (1D) power spectrum of $I, \chi_{B_{\text {pos }}}$, and $\phi_{\text {diff }}=\left|\theta_{\text {fil }}-\chi_{B_{\text {pos }}}\right|$ along the ridge crest. The power spectrum $P(s)$ of $Y(z)$ - one of the properties observed along a crest - is proportional to the square of its Fourier 
Table 1. Polarization properties derived from the BISTRO maps along 14 crests identified towards the NGC 6334 field. The crests 1 to 4 trace the ridge. The crests 5 to 14 correspond to the sub-filaments, for which in and out values are given (see Figs. 10 and 13 ).

\begin{tabular}{|c|c|c|c|c|c|c|c|c|c|c|}
\hline $\begin{array}{c}\text { Crest } \\
\# \\
(1)\end{array}$ & $\begin{array}{c}l_{\text {fil }} \\
{[\mathrm{pc}]} \\
(2)\end{array}$ & $\begin{array}{c}I_{\text {fil }} \pm \sigma_{I_{\text {fil }}} \\
{[\mathrm{Jy} / \text { beam] }} \\
(3)\end{array}$ & $\begin{array}{c}P I_{\text {fil }} \pm \sigma_{P I_{\text {fil }}} \\
{[\mathrm{mJy} / \mathrm{be}} \\
\text { (4) }\end{array}$ & $\begin{array}{l}\delta P I_{\text {fil }} \\
\text { 1] } \\
\text { (5) }\end{array}$ & $\begin{array}{r}P F_{\text {fil }} \pm \sigma_{P F_{\text {fil }}} \\
{[\%]} \\
\text { (6) }\end{array}$ & $\begin{array}{c}\delta P F_{\text {fil }} \\
(7)\end{array}$ & $\begin{array}{c}\chi_{B_{\mathrm{pos}}} \pm \sigma_{\chi_{B_{\mathrm{pos}}}} \\
{[\mathrm{deg}]} \\
(8)\end{array}$ & $\delta \psi_{\text {fil }}$ & $\begin{array}{c}\theta_{\text {fil }} \pm \sigma_{\theta_{\text {fil }}} \\
{[\mathrm{deg}]} \\
(10)\end{array}$ & $\begin{array}{c}\phi_{\text {diff }} \pm \sigma_{\phi_{\text {diff }}} \\
\text { [deg] } \\
(11)\end{array}$ \\
\hline 1 & 1.1 & $0.7 \pm 0.5$ & $18.0 \pm 3.2$ & 3.9 & $2.34 \pm 0.96$ & 0.50 & $92 \pm 21$ & 6 & $8 \pm 17$ & $83 \pm 27$ \\
\hline 2 & 0.7 & $3.3 \pm 2.6$ & $69.6 \pm 47.3$ & 3.9 & $1.83 \pm 0.98$ & 0.09 & $72 \pm 14$ & 1 & $55 \pm 14$ & $17 \pm 20$ \\
\hline 3 & 2.0 & $1.3 \pm 0.4$ & $33.7 \pm 9.1$ & 4.5 & $2.31 \pm 0.91$ & 0.30 & $153 \pm 26$ & 3 & $34 \pm 16$ & $61 \pm 31$ \\
\hline 4 & 3.0 & $3.2 \pm 0.7$ & $50.9 \pm 25.8$ & 3.9 & $1.66 \pm 0.78$ & 0.12 & $125 \pm 10$ & 2 & $26 \pm 12$ & $80 \pm 16$ \\
\hline 1 to 4 & 6.9 & $2.3 \pm 1.1$ & $33.1 \pm 14.5$ & 4.0 & $2.01 \pm 0.92$ & 0.20 & $121 \pm 22$ & $\overline{3}$ & $28 \pm 16$ & $87 \pm 28$ \\
\hline 5 in & 16 & $1.9 \pm 0.2$ & $16.3 \pm 7.0$ & 5.1 & $1.40 \pm 0.49$ & 0.38 & $78 \pm 37$ & 8 & $164 \pm 8$ & $85 \pm 38$ \\
\hline 5 out & 1.0 & $1.0 \pm 0.1$ & $11.4 \pm 4.3$ & 6.3 & $1.96 \pm 0.99$ & 1.18 & $108 \pm 40$ & 7 & $163 \pm 11$ & $55 \pm 41$ \\
\hline 6 in & & $2.0 \pm 0.2$ & $15.1 \pm 6.1$ & 5.2 & $1.32 \pm 0.54$ & 0.38 & $61 \pm 27$ & 10 & $24 \pm 6$ & $37 \pm 27$ \\
\hline 6 out & 1.7 & $0.4 \pm 0.2$ & $14.2 \pm 4.4$ & 7.5 & $4.06 \pm 2.30$ & 2.17 & $94 \pm 40$ & 8 & $47 \pm 13$ & $46 \pm 42$ \\
\hline 7 in & 10 & $0.8 \pm 0.6$ & $18.3 \pm 8.3$ & 4.6 & $1.96 \pm 0.68$ & 0.42 & $16 \pm 9$ & 7 & $70 \pm 11$ & $54 \pm 14$ \\
\hline 7 out & 1.0 & $0.3 \pm 0.1$ & $11.3 \pm 5.2$ & 5.4 & $4.47 \pm 2.29$ & 2.31 & $114 \pm 28$ & 6 & $33 \pm 20$ & $81 \pm 34$ \\
\hline 8 in & & $3.4 \pm 1.5$ & $28.6 \pm 9.7$ & 4.4 & $1.20 \pm 0.49$ & 0.19 & $77 \pm 31$ & 4 & $71 \pm 9$ & $6 \pm 33$ \\
\hline 8 out & 1.8 & $0.3 \pm 0.1$ & $15.3 \pm 5.5$ & 6.2 & $5.22 \pm 2.28$ & 2.41 & $167 \pm 19$ & 4 & $42 \pm 23$ & $54 \pm 30$ \\
\hline 9 in & 08 & $1.4 \pm 0.6$ & $49.3 \pm 37.3$ & 3.8 & $2.58 \pm 0.43$ & 0.20 & $103 \pm 7$ & 2 & $118 \pm 15$ & $14 \pm 16$ \\
\hline 9 out & 0.8 & $0.5 \pm 0.1$ & $11.4 \pm 3.9$ & 4.2 & $3.51 \pm 1.77$ & 1.36 & $177 \pm 22$ & 1 & $79 \pm 20$ & $82 \pm 30$ \\
\hline 10 in & & $1.7 \pm 0.8$ & $19.2 \pm 8.5$ & 4.2 & $1.64 \pm 0.37$ & 0.28 & $143 \pm 13$ & 6 & $168 \pm 21$ & $25 \pm 25$ \\
\hline 10 out & 1.2 & $0.3 \pm 0.1$ & $9.5 \pm 3.3$ & 4.7 & $4.60 \pm 2.48$ & 2.56 & $5 \pm 39$ & 5 & $162 \pm 18$ & $22 \pm 43$ \\
\hline 11 in & & $0.7 \pm 0.1$ & $18.1 \pm 5.4$ & 4.3 & $2.35 \pm 0.87$ & 0.59 & $132 \pm 19$ & 6 & $135 \pm 17$ & $3 \pm 26$ \\
\hline 11 out & 0.8 & $0.5 \pm 0.1$ & $14.5 \pm 5.4$ & 4.5 & $4.19 \pm 1.40$ & 1.32 & $106 \pm 18$ & 6 & $136 \pm 6$ & $29 \pm 19$ \\
\hline 12 in & & $0.9 \pm 0.1$ & $18.6 \pm 10.1$ & 4.4 & $2.47 \pm 0.88$ & 0.57 & $153 \pm 12$ & 6 & $5 \pm 30$ & $32 \pm 33$ \\
\hline 12 out & 1.4 & $0.9 \pm 0.2$ & $12.1 \pm 5.0$ & 4.8 & $1.96 \pm 0.80$ & 0.70 & $147 \pm 24$ & 6 & $106 \pm 28$ & $40 \pm 37$ \\
\hline 13 in & 11 & $0.8 \pm 0.2$ & $17.6 \pm 5.6$ & 3.8 & $1.64 \pm 0.60$ & 0.47 & $114 \pm 14$ & 6 & $86 \pm 20$ & $27 \pm 24$ \\
\hline 13 out & 1.1 & $0.6 \pm 0.1$ & $9.2 \pm 3.3$ & 4.3 & $2.43 \pm 1.41$ & 1.11 & $172 \pm 44$ & 5 & $160 \pm 14$ & $12 \pm 46$ \\
\hline 14 in & & $0.4 \pm 0.1$ & $15.3 \pm 4.9$ & 3.7 & $2.58 \pm 0.98$ & 0.73 & $119 \pm 20$ & 7 & $148 \pm 9$ & $29 \pm 22$ \\
\hline 14 out & 0.6 & $0.3 \pm 0.1$ & $14.0 \pm 3.5$ & 3.8 & $8.50 \pm 3.24$ & 2.51 & $167 \pm 7$ & 6 & $151 \pm 6$ & $16 \pm 9$ \\
\hline median in & 11 & $1.4 \pm 0.2$ & $18.3 \pm 8.3$ & 4.4 & $1.96 \pm 0.60$ & 0.42 & $114 \pm 19$ & 6 & $118 \pm 15$ & $29 \pm 26$ \\
\hline median out & 1.2 & $0.5 \pm 0.1$ & $12.1 \pm 4.4$ & 4.8 & $4.19 \pm 2.28$ & 2.17 & $147 \pm 28$ & 6 & $136 \pm 18$ & $46 \pm 37$ \\
\hline
\end{tabular}

Notes: The values given in this table are derived from BISTRO observations at a spatial resolution of 14". The row "1 to 4" corresponds to the full length of the ridge combining the crests from 1 to 4 . The last two rows "median in" and "median out" give the median values measured towards the sub-filaments "in" and "out" parts, respectively (see Fig. 13). Columns 3, 4, 6, 8, 10, and 11 give the median and their median absolute deviation per crests. These values are calculated along the ridge crests (for crests 1 to 4) and towards one-third of the "in" and "out" parts of the sub-filaments (c.f., Sect. 4.2). Columns 5, 7, and 9 give the statistical uncertainties. Columns: (1) Crest number. (2) Crest length. (3) Stokes I. (4) Polarized intensity. (6) Polarization fraction. (8) POS B-field orientation, $\chi_{B_{\mathrm{pos}}}$. To avoid artefacts introduced by the discontinuity in the distribution of angles around $0^{\circ}$ and $180^{\circ}$, which is the B-field orientation ambiguity inherent in polarimetric observations, we estimate two values of $\sigma_{\chi_{B \text { pos }}}$, one in the range $\left[0^{\circ}, 180^{\circ}\right]$ and another one in the range $\left[-90^{\circ}, 90^{\circ}\right]$. We then select the smallest value of $\sigma_{\chi_{B_{\mathrm{pos}}}}$ and the corresponding median $\chi_{B_{\mathrm{pos}}}$ value (mapped back to the $\left[0^{\circ}, 180^{\circ}\right]$ range when needed). (10) Orientation of the filament crest, $\theta_{\mathrm{fil}}$, measured on the Stokes I map. (11) Difference between the orientation of the POS B-field angle and the filament crest $\phi_{\text {diff }}=\left|\theta_{\text {fil }}-\chi_{B_{\text {pos }}}\right|$.

transform and can be expressed in 1D as

$P(s)=\frac{1}{L}|\tilde{Y}(s)|^{2}$

where $z$ is the spatial position along the crest (see, e.g., Figs. 11 and 12) and $s$ is the angular frequency. $\tilde{Y}(s)=\int Y(z) \exp ^{-2 i \pi s z} d z$ is the Fourier transform of $Y(z)$ and $L=\int d z$ is the total length of the studied crest.

Figure 16 shows the power spectra of $I, \chi_{B_{\text {pos }}}$, and $\phi_{\text {diff }}$ along crests 3 and 4 combined. The amplitudes of the power spectra are scaled so they can be plotted on the same panel.

No characteristic scales can been seen in the power spec-

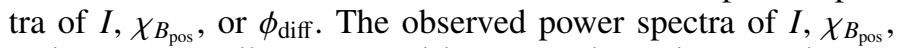
and $\phi_{\text {diff }}$ are well represented by power laws down to the angular resolution of the data $\left(14^{\prime \prime}\right.$ or $\left.s \sim 2.7 \operatorname{arcmin}^{-1}\right)$. We fit the observed power spectra with a power law function (where $s=2.7 \operatorname{arcmin}^{-1}$ is the highest frequency data point used for the fit). The best fit slope for $I$ is $\alpha_{I}=-1.74 \pm 0.27$. This value is compatible with the statistical results found by Roy et al. (2015) from the (1D) analysis of the column density along the crest of a sample of 80 subcritical/transcritical filaments observed by Herschel in the Gould Belt. The slopes of the power spectra of $\chi_{B_{\text {pos }}}$ and $\phi_{\text {diff }}$ are $\alpha_{\chi}=-1.33 \pm 0.23$ and $\phi_{\text {diff }}=-0.69 \pm 0.34$, $\sim 20 \%$ and $\sim 60 \%$ shallower than that of $I$, respectively. While the $\chi_{B_{\text {pos }}}$ distribution, which is defined in the range $\left[0^{\circ}, 180^{\circ}\right]$, may in some cases be affected by discontinuities around $0^{\circ}$ and $180^{\circ}$, that result from the B-field orientation ambiguity inherent in polarimetric observations, the power spectrum of $\chi_{B_{\text {pos }}}$ is not affected by such localised discontinuities, which would only contribute to the amplitude of the power spectrum at the smallest 
\%

$\cong$

$\Xi$

ฮี

.

$\frac{\sqrt[5]{0}}{7}$

t。

节

흘

实

$\stackrel{巳}{\Xi}$

○

동

ญ

ठ․

$\pm . \Xi$

ก

능

ปั.

E

:

$\stackrel{\Xi}{\Xi}$

จ

돕

$\forall$

ப்

䢔

는

늘 츜

ले

ชै

乙.

을

y

초

으

氖

을

岕

$\pm$

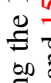

을 츨

幽

के

i 递

율

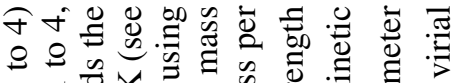

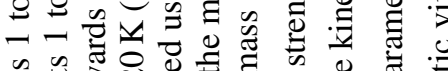

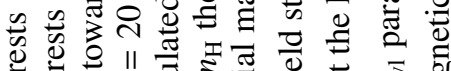

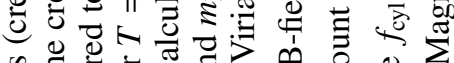

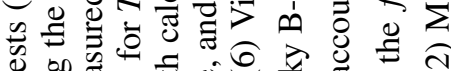
की \&.

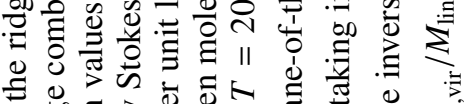

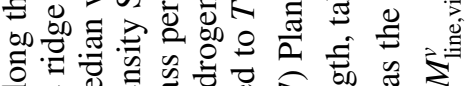

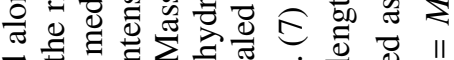
ठ

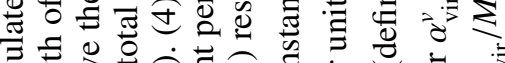

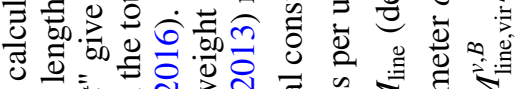

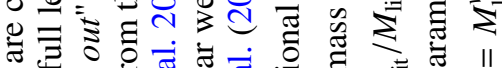

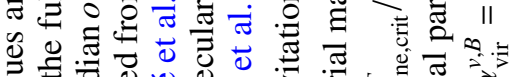

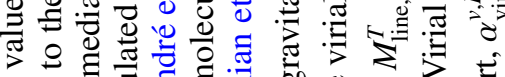

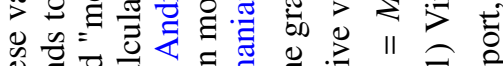

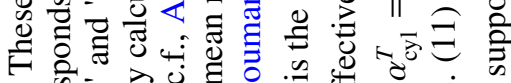

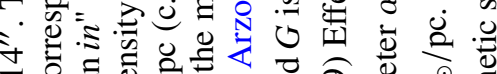

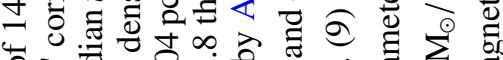

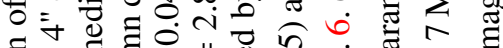
ธี

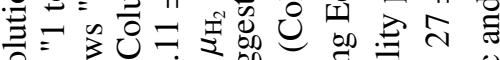
ช

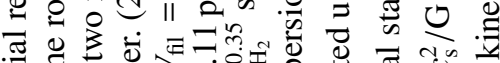

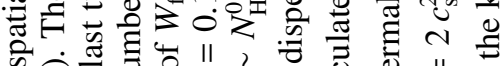

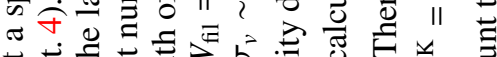

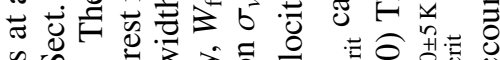

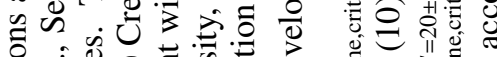

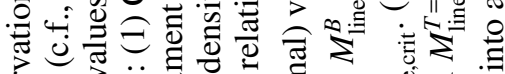

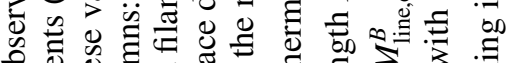

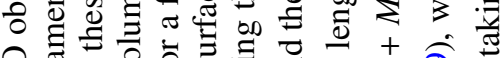
은 0 원

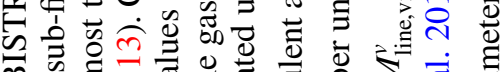

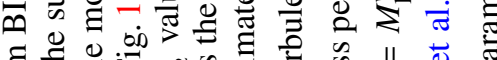

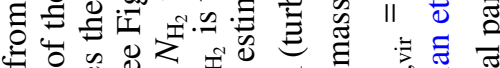

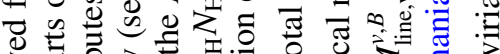

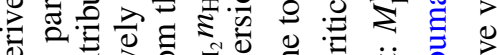
ठ ॠ

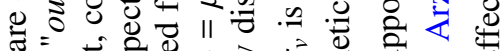

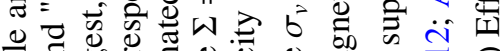

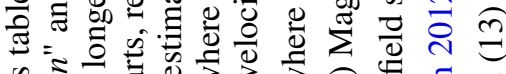

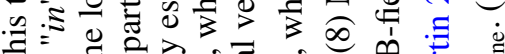

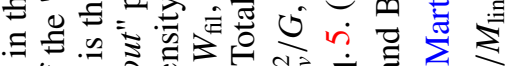
व

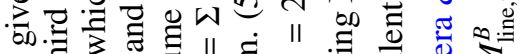

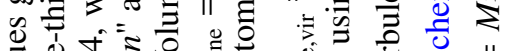

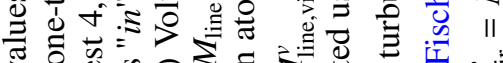

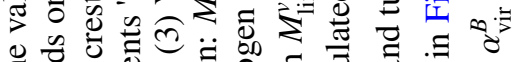

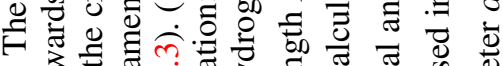
论

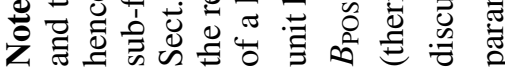




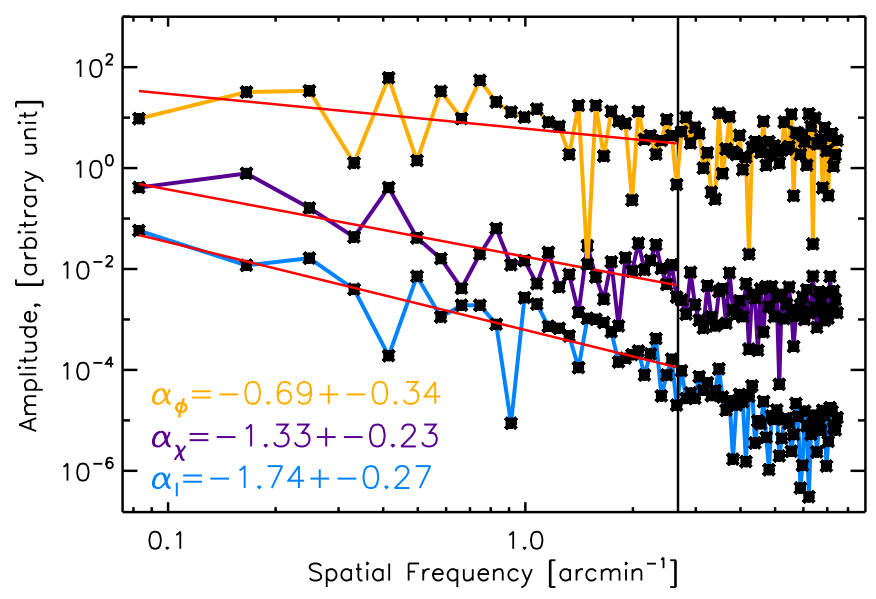

Fig. 16. Power spectra of $I, \chi_{B_{\mathrm{pos}}}$, and $\phi_{\text {diff }}$ in blue, purple, and yellow, respectively, observed along the crest ridge combining crests 3 and 4 (Fig. 10). The amplitudes are scaled to conveniently show the three power spectra on the same plot. The red solid lines indicate the best fit linear function (in log-log) to the observed power spectra. The derived best fit slopes are given in the bottom left of the plot, $\alpha_{I}$ in blue, $\alpha_{\chi}$ in purple, and $\alpha_{\phi}$ in yellow, for the power spectra of $I, \chi_{B_{\mathrm{pos}}}$, and $\phi_{\mathrm{diff}}$, respectively. The vertical black solid line indicates the $14^{\prime \prime}$ HPBW of the data at $850 \mu \mathrm{m}\left(s \sim 2.7 \mathrm{arcmin}^{-1}\right)$, which is also the highest frequency data point used to fit the power-law.

scales, i.e., at the largest spatial frequencies. Moreover, a clean power-law is also found for the power spectrum of $\theta_{\text {diff }}$ defined in the range $\left[0^{\circ}, 90^{\circ}\right]$, which is not affected by discontinuities at the edges of its interval of definition, suggesting that the power spectra of both $\chi_{B_{\mathrm{pos}}}$ and $\theta_{\text {diff }}$ are physically meaningful.

We checked whether the well defined power law shape of the observed power spectra could be obtained from uncorrelated emission of the properties along the filament crest. To do this test, we randomly reordered the observed values along the crest and derived the power spectra for 50 realisations of test-data. As can be seen on Fig. C.1, the power spectra for these test-data are not well represented by a power law and when fitted with a power law function the derived slopes are compatible with a flat spectrum. We hence conclude that the observed power spectrum slopes, $\alpha_{I}, \alpha_{\chi}$, and $\alpha_{\phi}$, are a result of correlated fluctuations along the filament crest and they can be used to infer physical properties of the filaments. We discuss physical interpretations of this proposed power spectrum analysis in Sect. 6.4.

\section{Discussion}

\subsection{Comparing the observational results of NGC 6334 with other regions observed as part of BISTRO}

NGC 6334 , at $1.3 \mathrm{kpc}$, is the first high-mass star-forming region at a distance larger than that of the Gould Belt clouds $(\sim 500 \mathrm{pc})$ that has been analyzed as part of the BISTRO survey. These observations reveal the B-field structure at a resolution of $\sim 0.1 \mathrm{pc}$ towards $\mathrm{a} \sim 10 \mathrm{pc}$-long ridge and a parsec-scale clump-hub structure. The results of the analysis of our data show similarities and differences with the regions already analysed as part of the BISTRO survey.

Figure 17 presents a comparison of the $P F$ values against $I$ observed towards three regions: NGC 6334 (this work), NGC 1333 (Doi et al. 2020), and Oph-A (Kwon et al. 2018; Pattle et al. 2019). The values of $P F$ span very similar ranges from $\sim 20 \%$ down to $\sim 0.2 \%$. In the three regions, we observe an overall decrease of $P F$ for increasing $I$ (with similar upper-envelope slopes). The range in $I$ and column density observed towards these three regions are also very similar from $\sim 10^{21} \mathrm{~cm}^{-2}$ to $\sim 10^{23} \mathrm{~cm}^{-2}$ (the lower value being due to the selection of data; in this case $S N R(I)>20$ for all three regions). In NGC 6334, values up to $\gtrsim 10^{24} \mathrm{~cm}^{-2}$ are measured (towards the two corehubs) as a hint of the extreme conditions of this region forming high-mass stars and stellar clusters compared to the other Gould Belt clouds. All three regions show a large scatter in $P F$ for a given value of $I$ as well as a large scatter in $I$ for a given value of $P F$.

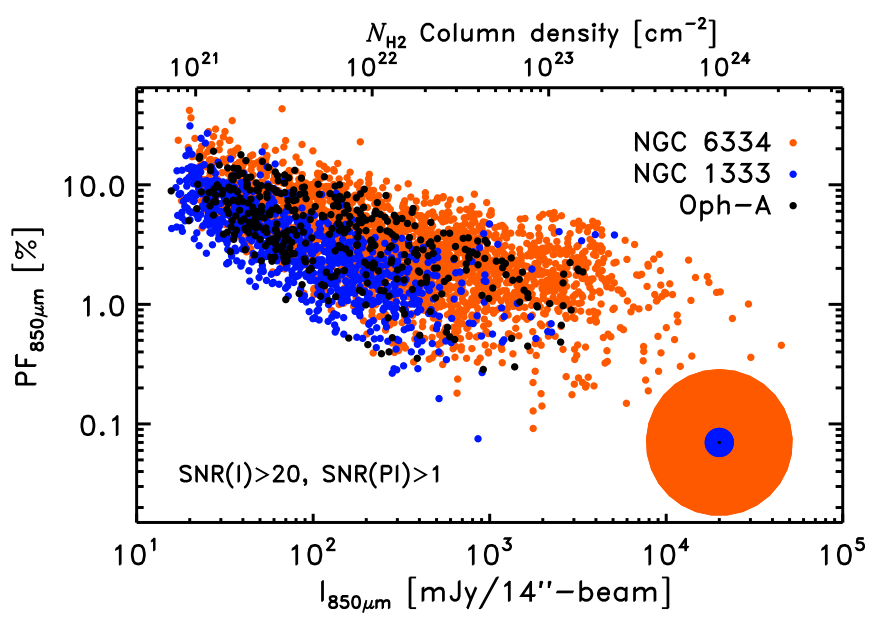

Fig. 17. Comparison plot of $P F$ as a function of $I$ derived from BISTRO observations in three different regions: NGC 6334 in orange (this work, similar to the middle panel of Fig. 8), NGC 1333 in blue (Doi et al. 2020), and Oph-A in black (presented in Kwon et al. 2018, and also Furuya et al., in prep.). The concentric filled circles on the bottom right of the plot indicate the relative sizes of the regions corresponding to about $10 \mathrm{pc}, 2 \mathrm{pc}$, and $0.2 \mathrm{pc}$ in total extent, for NGC 6334, NGC 1333 , and Oph-A, respectively. The angular resolution of the BISTRO data are $0.09 \mathrm{pc}, 0.02 \mathrm{pc}$, and $0.01 \mathrm{pc}$ in physical scales for NGC 6334, NGC 1333 , and Oph-A, at the distance of $1.3 \mathrm{kpc}, 299 \mathrm{pc}$, and $150 \mathrm{pc}$, respectively.

These similarities are all the more intriguing considering that the three regions are at different distances from us. The closest is Oph-A at a distance of just 150 pc, then NGC 1333 at 299 pc, and the furthest is NGC 6334 at $1.3 \mathrm{kpc}$. The physical sizes of the observed fields span 2 orders of magnitude from $\sim 0.2 \mathrm{pc}$, to $\sim 2 \mathrm{pc}$, and $\sim 10 \mathrm{pc}$ (in total extent), for the nearest to the furthest regions (see Fig. 17). The observed similarities despite the range of scales, could suggest that the physical properties of the dust grains emitting the linearly polarized light are comparable in all these regions and that the integration along the magnetized LOS (towards different scales) affects the observed emission in a similar way. This simple comparison could also suggest that the self-similar description of the physical processes in a possibly fractal magnetized ISM has an imprint in the dust polarized thermal emission. We refer the readers to future publications presenting the detailed comparison of different regions observed as part of the BISTRO survey.

In all three regions, the prevailing POS B-field angle observed at $14^{\prime \prime}$ is compatible with the orientation derived at $5^{\prime}$ from Planck data and starlight polarization. The high-resolution observations with BISTRO, however, reveal the complex smallscale structures of the B-field. This B-field spans a wide range of POS angle with no characteristic orientation when integrated 
over the whole region. Detailed inspection of the maps, however, shows the locally ordered B-field geometries in connection with the underlying intensity structure, i.e., the line mass distribution. In NGC 1333 for example, the POS B-field is observed to be ordered along each filament but its relative orientation with respect to the filament crests varies from one filament to the other. This variation of the relative orientation between $\chi_{B_{\mathrm{pos}}}$ and $\theta_{\mathrm{fil}}$ is arguably due to projections effects (cf., the analysis presented in Doi et al. 2020).

In NGC 6334, $\chi_{B_{\mathrm{pos}}}$ varies significantly along the different filament crests where we can see the imprint of the formation, evolution, and interaction of the sub-filaments, ridge, and hubs. With these observations, we can also trace the effects of feedback from past and on-going high-mass star formation, such as in the surroundings of the sources from I to V indicated on Fig. 1top-left. We qualitatively discuss these two aspects below and leave more detailed analysis to future works (e.g., Tahani et al., in prep.).

\subsection{The B-field structure of a high-mass star forming hub-filament system}

The B-field structure is on average perpendicular to the northern section (crest 4) of the main ridge of NGC 6334, which would suggest a formation process compatible with the theoretical model proposed by Inoue et al. (2018), where a filament is formed by the convergence of a flow of matter along the B-field lines hence forming a filament perpendicular to these lines (see also Inoue \& Fukui 2013; Vaidya et al. 2013; Arzoumanian et al. 2018; Bonne et al. 2020). In this scenario, the flow of matter would be initially generated by the bending of the ambient Bfield lines induced by an interstellar shock compression. Such a bent structure of the B-field lines around elongated molecular clouds is compatible with the observational findings of Tahani et al. (2018) and Tahani et al. (2019). Large scale CO molecular line observations have suggested that NGC 6334 may be located at the shock front of the collision of two clouds (Fukui et al. 2018).

The network of sub-filaments surrounding the ridge is connected to it in a roughly perpendicular orientation (see Fig. 13). The POS B-field is mostly perpendicular (or has a random orientation with respect) to the sub-filaments in their outer parts. In the inner parts, where the sub-filaments merge with the clumphub, the relative orientation between the sub-filaments and the POS B-field angle becomes mostly parallel (see Figs. 13 and 14). This trend has also been found recently towards the G33.92+0.11 (Wang et al. 2020) and Serpens South (Pillai et al. 2020) hubfilament systems. This finding suggests that the sub-filaments may be formed orthogonal to the ambient B-field and then merged with the ridge, forming a clump-hub structure, due to, e.g., shock compression events, interstellar turbulence, or under the gravitational attraction by the ridge itself. Indeed, the whole region, i.e., the clump-hub and the sub-filaments (crests 5 to 10,13 , and 14), is virially bound with a total mass of $\sim 7700 \mathrm{M}_{\odot}$ and total virial masses of $\sim 300-1000 \mathrm{M}_{\odot}$, assuming a spherical geometry with an effective radius of $\sim 1 \mathrm{pc}$ and $\sigma_{v} \sim 0.5-1.0 \mathrm{~km} \mathrm{~s}^{-1}$.

In addition to a large-scale longitudinal velocity gradient observed in $\mathrm{HCO}^{+}$along the ridge, some of the sub-filaments (as identified in Fig. 10) exhibit longitudinal velocity gradients perpendicular to the ridge (see Fig. 2 of Zernickel et al. 2013). ALMA high-resolution molecular line observations of dense gas show also multiple velocity components along a section of the southern part of crest 4 (Shimajiri et al. 2019), that may be tracing dynamical interactions between the ridge and the surrounding sub-filaments. These velocity gradients may be tracing matter flowing onto the ridge/clump-hub or the merging of the sub-filaments with the ridge. The B-field structure at the tip of the sub-filaments evolves under the influence of the gravitational potential of the clump-hub, while the B-field in the outer part of the sub-filaments still keeps the initial orientation, which better reflects the physical process leading to their formation. The variation of the B-field structure along the sub-filaments from their outer parts to their inner parts may be tracing and be coupled to these local velocity flows of matter in-falling onto the clumphub, as also suggested by MHD simulations (Gómez et al. 2018).

Along crest 3 of the ridge, the B-field orientation varies from being mostly perpendicular to parallel to the crest (Fig. 12). Comparing with the velocity field derived from $\mathrm{HCO}^{+}(3-2)$ molecular line data (Zernickel et al. 2013), this variation of the B-field structure may be induced by longitudinal motions along the crest, possibly by the gravitational potential of source III, a young cluster with high-mass stars. This variation of $\chi_{B_{\mathrm{POS}}}$ observed towards the source III may also be due to the effect of feedback from the expansion of the UCH II region associated with this young high-mass star-forming site.

Figure 18 shows the variation of the polarization properties as two sub-filaments (crests 5 and 6) merge into a single filament (in projection at least) connected to the clump-hub. Transverse profiles of $I$ and $P I$ across the two separated crests 5 and 6 shows two peaks in $I$ and is flat in PI (red curves in Fig. 18). The two peaks in the $I$ profile show larger values and their separation gets smaller as the distance to the clump-hub shrinks. When the two crests are observed to merge (corresponding to the blue segments in Fig. 18-Left), a single peak in $I$ is seen, with a value $\sim 4$ times larger compared to the peak intensity when the two crests were separated. This increase in $I$ towards the crests of the merged-filaments is larger than a simple addition of the intensity of the two separated-filaments, suggesting 1) this merging is not due to projection effects and integration along the LOS of two physically independent filaments, but 2) there is an additional efficient infall of matter onto the merged filaments (resulting in an increase of the density). We might be witnessing here the precursor of a core-hub. The $P I$ profile across the merged filaments shows a dip (seen also in $P F$ ) at the position of the peak in $I$. The POS B-field angle across these two filaments also changes and is observed to be perpendicular to the crest after they merge, while the B-field lines are bent on both sides of the crest in a horseshoe pattern (Figs. 4 and 18). This correlation between the variation of the POS B-field structure and the dip in $P I$ and $P F$ may be tracing depolarization of the polarized emission due to the integration along the LOS or within the beam of unresolved B-field structures. Similar variations of the polarization properties can be seen along crest 8 , which seems to be connected to the core-hub I(N) (see Figs. 4 and 13). These variations are also associated with an increase of the dispersion of $\chi_{\text {POS }}$ (c.f., Fig. 7) suggesting the change of the geometry of the B-field as the subfilaments merged with the clump- and core-hubs.

As can be seen in Table 2 and Fig. 15, the ridge and most of the sub-filaments of NGC 6334 are thermally supercritical with $\alpha_{\text {cyl }}^{T}<1$. Towards the ridge, both turbulent $\alpha_{\text {vir }}^{v}$ and magnetic $\alpha_{\text {vir }}^{B}$ virial parameters $<1$, suggesting that when taken separately the thermal, turbulent, or magnetic energy alone cannot support the ridge against gravity. Towards the sub-filaments, $\alpha_{\text {vir }}^{v}$ decreases and $\alpha_{\text {vir }}^{B}$ increases from their outer parts to their inner parts, suggesting stronger role of the gravity and magnetic field in the inner parts of the sub-filaments when they merge with the clump- 

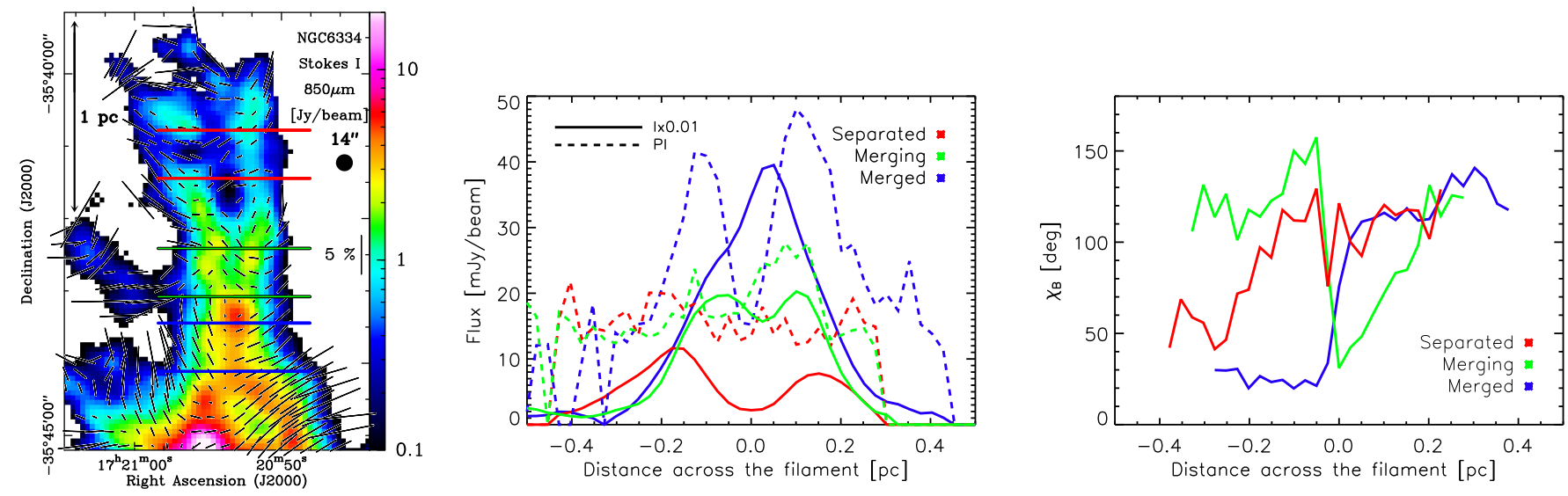

Fig. 18. Left: Stokes $I$ map of the northern region of NGC 6334 towards the sub-filaments 5 and 6 (see Fig. 10). The black lines correspond to $\chi_{B_{\mathrm{POS}}}$ and their lengths are proportional to $P F$. On the right hand side, a vertical line indicates the $5 \% P F$ scale. The black filled circle indicates the $14^{\prime \prime}$ beam. The horizontal blue, green, and red lines show the $(0.8 \mathrm{pc})$ cuts used to derive the profiles shown on the middle and right panels, for the merged, merging, and separated profiles, respectively. Middle: Three transverse cuts, from East to West, where the sub-filaments are still separated (red), where they are merging (green), and where they are merged (blue) and not distinguishable any more as two peaks in Stokes $I$. Each profile has been averaged vertically between the two lines of the same colour indicated on the left hand side plot. The solid and dashed line profiles show Stokes $I$ and $P I$, respectively. We see a drop of $P I$ towards the peak in $I$ when the two filaments merge, that may be due to depolarization via beam-smearing of the B-field where the two sub-filaments merge. Right: Same as the middle panel for the variation of $\chi_{\mathrm{B} p o s}$ across the two sub-filaments.

hub. When all three main pressure forces are considered simultaneously, $\alpha_{\text {vir }}^{v, B} \gtrsim 1$, implying that while gravity is important in the evolution of and the interactions between the NGC 6334 filaments, the magnetic field as well as kinetic support play a key role in their overall energy balance.

\subsection{Impact of the star formation feedback on the observed polarization properties}

A main difficulty in the interpretation of polarization observations is that dust polarized emission depends on 1) the dust grain properties (size, shape, composition), 2) the efficiency of grain alignment with the local B-field, and 3) on the magnetic field structure (mean orientation with respect to the LOS and dispersion on the POS and along the LOS). In addition, the observed emission is integrated along the LOS and averaged within the observational beam. Thus understanding the origin of the variation of the polarization properties, e.g., the relation between the polarization fraction and the intensity (column density), cannot be gained without taking into account these effects simultaneously. For example, large $P F$ values require well aligned grains and ordered B-field structures with optimal orientations with respect to the LOS, while low $P F$ values may be due to a loss of grain alignment, variation of the grain properties, or a change of the B-field configuration. At the observed densities, the alignment efficiency of dust grains with the local B-field lines is independent from the absolute B-field strength. In addition, alignment itself is considered instantaneous given the dynamical timescales of the molecular cloud even when there are interactions and shock compressions between different structures/filaments (Hildebrand 1988; Voshchinnikov 2012; Hoang \& Lazarian 2016). The observed $P F$ values, however, may be affected by the B-field strength due to the increase or decrease of depolarization from a turbulent or ordered B-field structure, respectively.

As mentioned earlier, NGC 6334 is actively forming stars and stellar clusters. The dense and cold molecular gas is affected by feedback from the high-mass stars formed along and next to the ridge. This region is hence an excellent laboratory to study the effect of feedback on the polarized dust emission properties and the B-field structure. While we refer the reader to a companion paper (Tahani et al. in prep.) for a detailed analysis, we give below a qualitative overview on the impact of the expanding H II regions on the surrounding $\mathrm{B}$-field and polarized emission.

As an example of the impact of feedback, we note the POS B-field structure bends along the dense shell around the $\mathrm{H}$ II region corresponding to source II, where the expansion of the H II region has swept up most of the local dust and has created a cavity of submm continuum emission seen in our observations (c.f., Fig. 19). This expansion likely influenced the pre-existing B-field structure, and the field was dragged along with the swept-up material. The cloud was reshaped into the now-observed shell with enhanced polarized emission due to an ordered B-field structure curved along the compressed shell (see, e.g., Tang et al. 2009; Planck Collaboration Int.XXXIV 2016, for similar discussions). A similar feedback effect may be seen towards the shell associated with the HII region to the South of source IV, albeit with a more complicated B-field structure and polarization pattern. Detailed 3D modelling is needed to understand the origin of these observed emission. This study will be partly addressed in Tahani et al. (in prep.).

The clump-hub shows another example of the impact of the feedback. Figure 20-top shows a scatter plot of $P F$ as a function of $I$ towards the clump-hub (black dashed ellipse in Fig. 19). All the data points towards the clump-hub have $S N R(I)>25$ and $S N R(P I)>3$. We see an overall decreasing trend of $P F$ as a function of $I$ (c.f., Fig 8). A decrease in $P F$ with $I$ (or $N_{\mathrm{H}_{2}}$ ) has been reported in previous studies and ascribed to the loss of grain alignment efficiency or the random component of the magnetic field (e.g., Jones et al. 1992; Whittet et al. 2001, 2008; Jones et al. 2015; Planck Collaboration Int. XIX 2015). Notably, the observed $P F$ versus $I$ data points from the clump-hub are not well described by a single mean power law. We can see that the linear fit $\log P F$ versus $\log N_{\mathrm{H}_{2}}$ for the data points with $N_{\mathrm{H}_{2}}<2 \times 10^{22} \mathrm{~cm}^{-2}$ provides a best fit slope of $-0.72 \pm 0.09$, while for the data points with $N_{\mathrm{H}_{2}}>2 \times 10^{22} \mathrm{~cm}^{-2}$ the fitted slope $-0.14 \pm 0.04$ is much shallower. A fit to the upper envelope of the distribution yields a slope of $-0.29 \pm 0.05$. All of these slopes 


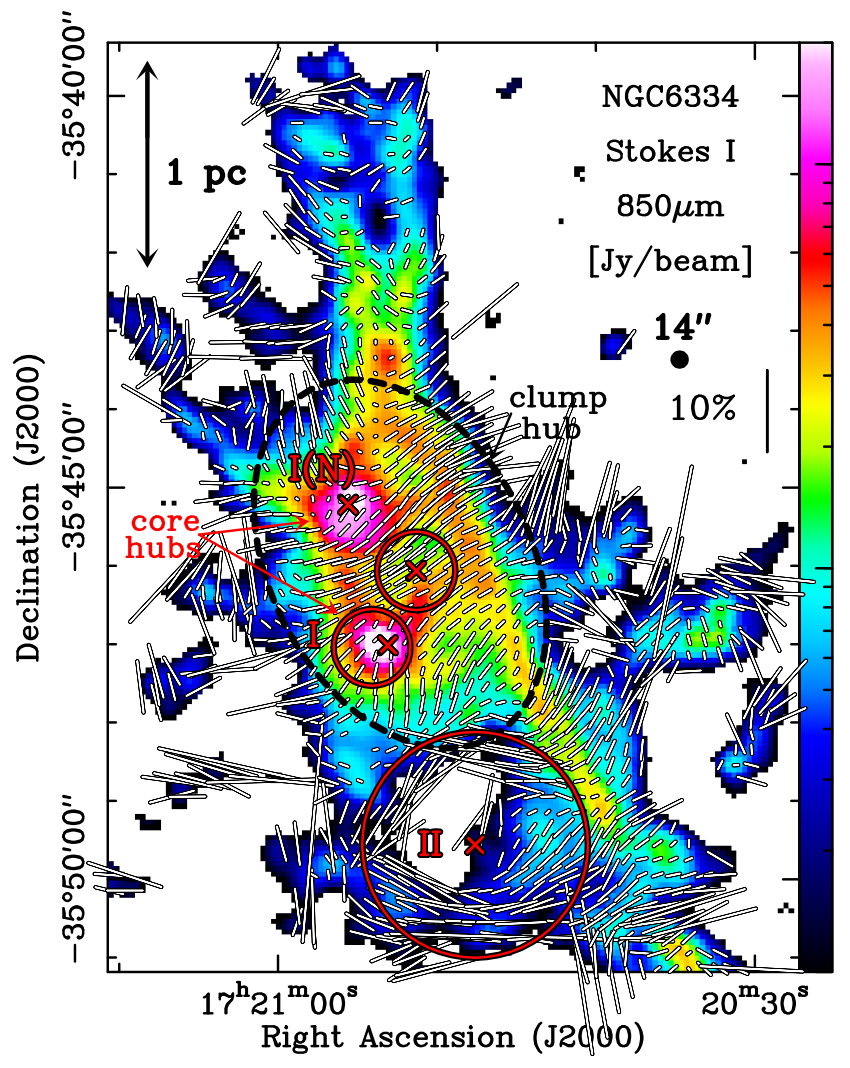

Fig. 19. Zoom-in of the northern region of NGC 6334 (same as Fig. 13). The white lines show $\chi_{B_{\mathrm{POS}}}$ and their lengths are proportional to $P F$. On the right hand side, a vertical line indicates the $10 \% P F$ scale. The black filled circle indicates the $14^{\prime \prime}$-beam. The black dashed ellipse shows the clump-hub. The two core-hubs I and I(N) are also indicated. The crosses and circles are the same as in Fig. 1-top-left.

are shallower than the $P F \propto N_{\mathrm{H}_{2}}^{-1}$ relation expected from the progressive loss of grain alignment expected in the framework of radiative torques (RAT) grain alignment theory due to the attenuation of the interstellar radiation field with increasing column density (e.g., Cho \& Lazarian 2005; Lazarian 2007; Lazarian \& Hoang 2007; Hoang \& Lazarian 2014).

For these high column densities $N_{\mathrm{H}_{2}} \gg 10^{22} \mathrm{~cm}^{-2}$, the observed shallower slopes may be tracing an increase of the grain alignment efficiency due to the enhanced anisotropic radiation from newborn stars embedded in the clump-hub. Indeed, according to the RAT theory, anisotropic radiation from stars embedded in high-column density clumps increases the efficiency of grain alignment (Lazarian \& Hoang 2007; Lee et al. 2020; Hoang et al. 2020), which may result in the observed shallower slopes (see also Pattle et al. 2019). Moreover, Hoang et al. (2019) showed that when the intensity of the radiation from the embedded stars is large, the polarization fraction decreases due to the removal of large grains. We may be witnessing this effect towards the core-hubs (red data points in Fig. 20-top), which show a steep decrease of $P F$ for $N_{\mathrm{H}_{2}} \gtrsim 10^{24} \mathrm{~cm}^{-2}$. This effect may also be seen in the bottom panel of Fig. 20, which traces the variations of $P F$ and $I$ across the two core-hubs. Towards the older source I, a trough in $P F$ is associated with an increase in Stokes $I$. Towards the youngest source $\mathrm{I}(\mathrm{N})$, however, there is a spatial shift between the position of the dip in $P F$ and the peak in $I$. Lower $P F$ values are observed towards the edge of this latter source compared to larger $P F$ values towards its high column density centre. In the cut towards the source I, the emission from the
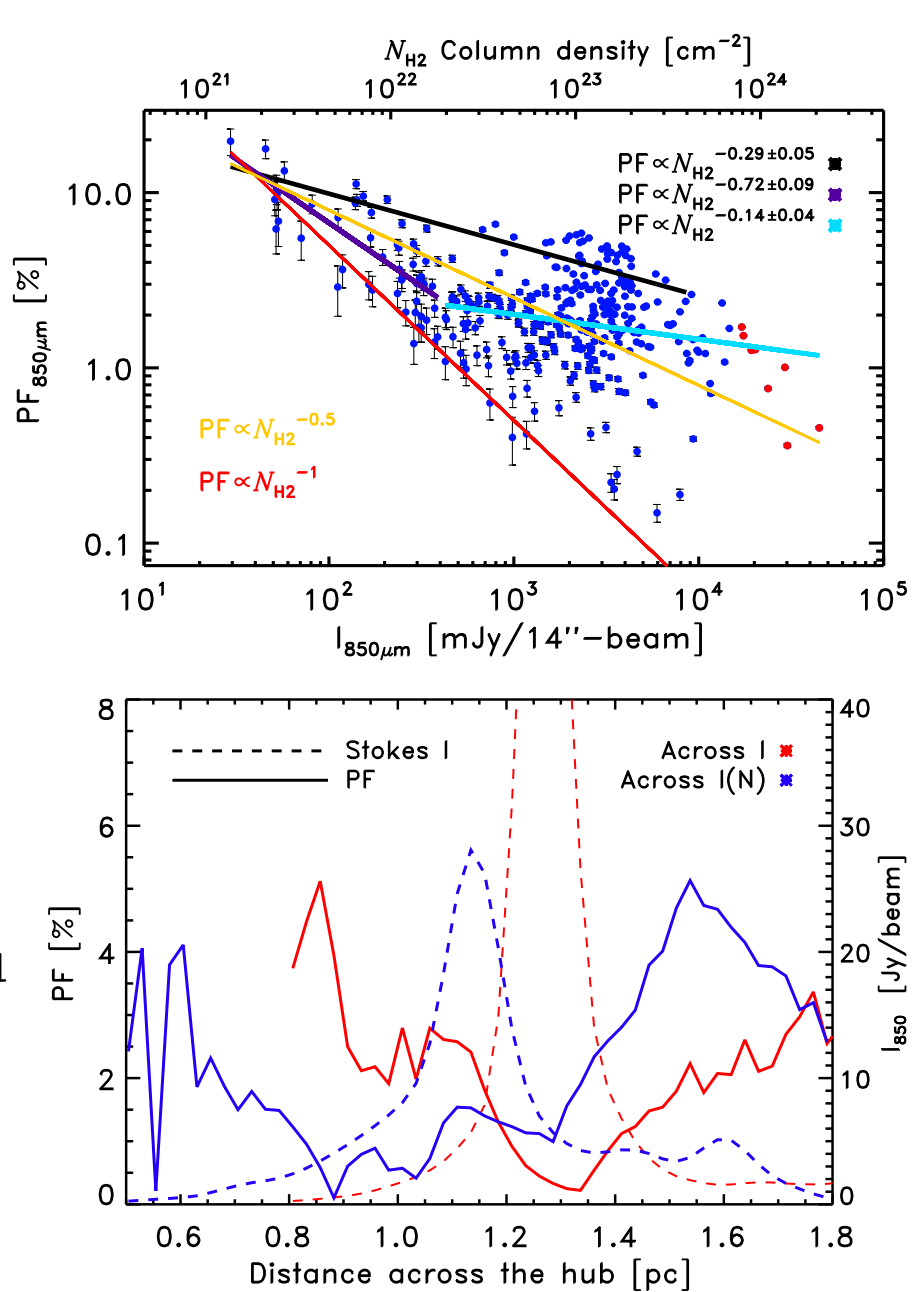

Fig. 20. Properties observed towards the clump-hub (black dashed ellipse in Fig. 19). Top: $P F$ as a function of $I$ for $S N R(I)>25$ and $S N R(P I)>3$. The vertical small black lines show the statistical uncertainties on $P F$. The black, purple, and cyan lines indicate the best linear fits to the upper $95 \%$ percentiles of the distribution, the data points with $N_{\mathrm{H}_{2}}<2 \times 10^{22} \mathrm{~cm}^{-2}$, and the data points with $N_{\mathrm{H}_{2}}>2 \times 10^{22} \mathrm{~cm}^{-2}$, respectively. The best fit slopes are indicated on the plot. For comparision, the yellow and red lines indicate slopes of -0.5 and -1 , respectively. Bottom: Variation of $P F$ (solid lines) and $I$ (dashed lines) for two horizontal cuts from East to West, crossing the star forming regions I and $\mathrm{I}(\mathrm{N})$ in red and blue, respectively. The peak in $I$ of the blue dashed profile at $\sim 1.6 \mathrm{pc}$ corresponds to the position of the ridge (crest 4 ).

ridge (crest 4 ) can also be seen (at $\sim 1.6 \mathrm{pc}$ from the $x$-axis in Fig. 20) which interestingly is not associated with a drop in $P F$, but with large $P F$ values. The observed POS B-field towards the crest 4 embedded in the clump-hub has an ordered structure, which may be also contributing to the observed large $P F$ values. The slopes derived from the $P F$ versus $I$ plot in Fig. 20-top are also shallower than the -0.5 slope expected from a purely turbulent B-field (Jones et al. 2015; Andersson 2015), consistent with the observed ordered B-field structure towards the clump-hub (c.f., e.g., Fig. 19).

In summary, the polarization features observed towards NGC 6334 may result from the impact of star formation feedback. Such features include: 1) the re-organization of the structure of the B-field on the scale of $\mathrm{H}$ II regions (within the compressed shells) where the mean B-field orientation is reoriented closer to the POS, 2) an increase of the B-field strength, ordering its structure and reducing depolarization due to integration of emis- 


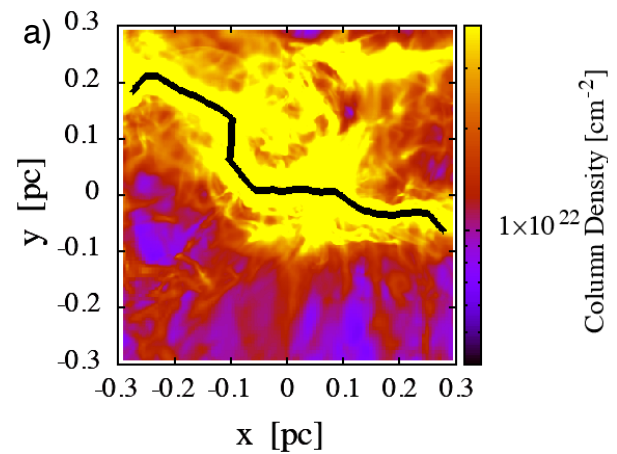

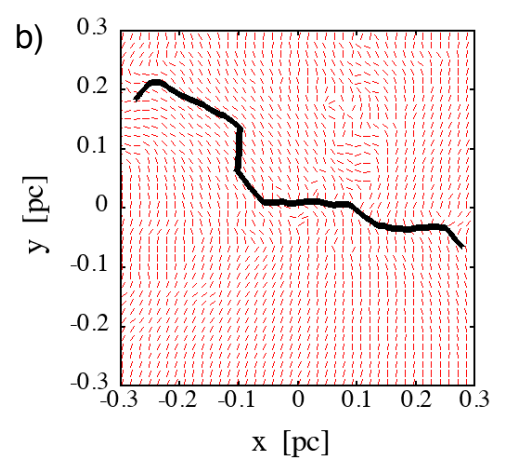

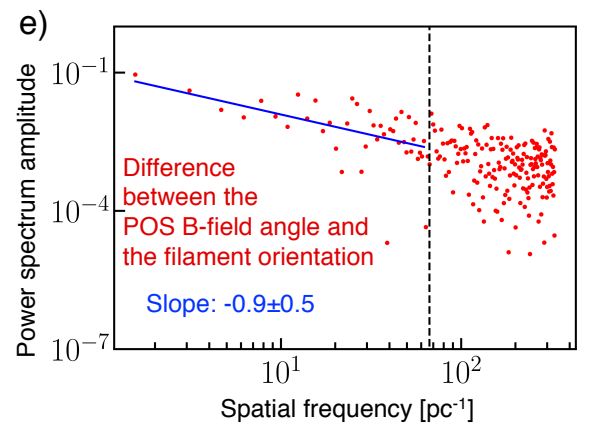

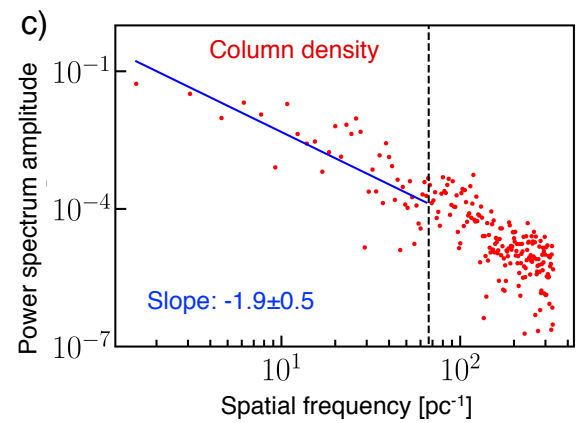

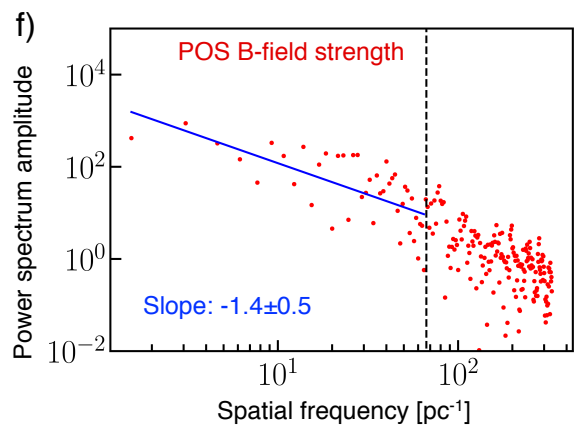

Fig. 21. Results derived from the MHD numerical simulation presented by Inoue et al. (2018). The selected snapshot corresponds to $t=0.7 \mathrm{Myr}$ after the filament formation by shock compression. The mass per unit length of the filament at this epoch is $\sim 120 \mathrm{M}_{\odot} / \mathrm{pc}$. a) Column density map of the filament. The black curve shows the crest of the filament traced with FilFinder (Koch \& Rosolowsky 2015). b) The small red lines indicate the POS B-field angle towards the same region shown in panel a). The black curve is the crest of the filament. From c) to f) Power spectra of the column density, the POS B-field angle, the difference of the angle between the POS B-field orientation and the filament orientation, and the B-field strength, respectively. The slopes derived from the power law fits (shown in blue) to each of the power spectra are indicated on the panels. The vertical black dashed line indicates the highest frequency data point used to fit the power-law corresponding to 10 computational cells.

sion along the LOS and within the beam, 3) an increase of the efficiency of grain alignment with the local B-field due to an enhanced local radiation field from the newborn high-mass stars, and 4) a drop of polarization fraction towards the densest corehubs due to the removal of large grains from stronger stellar feedback or a more tangled and unresolved configuration of the B-field.

\subsection{Understanding the origin of the slope of the B-field power spectrum}

A recent analysis of the column density variations along the crest of a sample of filaments observed by Herschel in three star-forming clouds indicates that the 1D column density power spectra of these filaments are well defined by a power law function (down to Herschel resolution of $\sim 0.02 \mathrm{pc}$ ) with a mean slope of $-1.6 \pm 0.3$ (Roy et al. 2015). This observed power law slope is compatible with the theoretical value suggested by Inutsuka (2001) close to twenty years ago to explain the origin of the Salpeter (1955) power-law slope of the IMF at the highmass end. More recently, in an attempt to investigate the origin of the observed angular momentum of star-forming cores, Misugi et al. (2019) proposed a new theoretical model based on the filament velocity power spectrum. This model suggests that cylindrical filaments characterized by a 1D Kolmogorov velocity power spectrum slope of $-5 / 3$ will fragment into cores showing a distribution of angular momentum compatible with the observations.

The results presented by Roy et al. (2015) and Misugi et al. (2019) suggest that the column density (line mass) and velocity variations/fluctuations along a filament may help to illuminate the origin of the CMF/IMF shape and the properties of the cores (e.g., angular momentum) formed from filament fragmentation. Since the gas and the magnetic field are dynamically well coupled in the ISM (i.e., frozen-in conditions), the magnetic field power spectrum should be related to the density and velocity power spectra and this link would depend on the physical processes resulting in the formation of the ISM structures. Thus, the observational determination of the power spectrum of the magnetic field configuration could provide valuable information on the origin of the formation of these structures and the link between the kinetic, magnetic, and gravitational energies at play in the ISM (see also Li \& Burkert 2018 for a theoretical approach linking the power spectra of the density and magnetic energy fields in the ISM).

Here we present for the first time the power spectrum of the B-field POS angles $\chi_{B_{\mathrm{pos}}}$ observed along the NGC 6334 ridge (cf., Fig. 16). We show that the power spectrum of $\chi_{B_{\text {pos }}}$, similarly to the power spectrum of $I$, can also be well represented with a power law function and no characteristic scales are detected down to the resolution of the data. The slope of the power spectrum of $\chi_{B_{\text {pos }}}-1.33 \pm 0.23$ is measured to be $\sim 20 \%$ shallower than that of $I(-1.74 \pm 0.27$, cf., Fig. 16). We also present the power spectrum of the difference of angle between $\chi_{B_{\mathrm{pos}}}$ and the ridge crest orientation; $\theta_{\text {diff }}$ defined in the interval $\left[0^{\circ}, 90^{\circ}\right]$. This latter power spectrum has a slope of $-0.69 \pm 0.34$, shallower than the power spectrum slope of $I$ and $\chi_{B_{\mathrm{pos}}}$.

We compare this observational result with the power spectrum of the magnetic field angle along a supercritical filament formed in the MHD numerical simulation of Inoue et al. (2018). Figure 21 presents the power spectra of the column density, the POS B-field angle, and the difference between the POS B- 
field angle and the filament orientation derived from the threedimensional data cubes of this simulation. The slopes of the power spectra of the different quantities derived from the observations and the simulations are in agreement. This similarity suggests that the observed column density (line mass) and B-field fluctuations along the star forming NGC 6334 filament may be inherited from the filament formation process and have a coupled evolution due to, e.g., the accretion of matter and fragmentation under the interplay of the turbulent, gravitational, and B-field energies as incorporated in this MHD simulation. We also derive the power spectrum of the POS B-field intensity along the filament from the MHD simulation. This latter power spectrum has a power law slope similar to that of the POS B-field angle power spectrum, both $\sim 20 \%$ shallower than that of $I$ (c.f., Fig. 21).

Following these preliminary results, we suggest that understanding the theoretical link between the power spectra of column density, velocity, and magnetic field (both angle and strength) and its evolution in time may lead to a new and independent method to infer the B-field strength from polarization observations, which do not provide a direct measurement of the B-field strength.

Here we presented the analysis of the power spectra of different quantities towards a single observed filament and a single simulated filament. Further detailed analysis of the column density and B-field fluctuations along statistical samples of both observed and simulated filaments are necessary to understand better the underlying physical processes captured by the observations.

\section{Summary and conclusions}

In this paper, we present the first BISTRO results of the JCMT/SCUBA-2/POL-2 observations at $850 \mu \mathrm{m}$ towards the NGC 6334 high-mass star-forming hub-filament network. Our main results may be summarized as follows:

- BISTRO observations provide for the first time the submm polarization properties at high angular resolution (14" or $0.09 \mathrm{pc}$ at $1.3 \mathrm{kpc}$ distance) and high $\operatorname{SNR}(\operatorname{SNR}(I) \gg 25$ and $S N R(P I) \gg 3)$ towards the $10 \mathrm{pc} \times 2 \mathrm{pc}$ hub-filament network hosting a 10 pc-long ridge, sub-filaments, and core-hubs where stellar clusters and high-mass stars are forming (Figs. 1, 2, 3, and 4).

- The observations show a positive correlation between the polarized intensity $P I$ and the total intensity $I\left(P I \propto I^{0.65 \pm 0.02}\right)$, where both $P I$ and $I$ span $\sim 3$ orders of magnitude in polarized and total emission, respectively (Fig. 8-left). This correlation, however, exhibits a scatter by more than one order of magnitude in $P I$ for $I$ values spanning $\sim 3$ orders of magnitude. The observations show an overall negative correlation between the polarization fraction $P F$ and $I$ over $\sim 3$ orders of magnitude in $P F$ with $P F \propto I^{-0.35 \pm 0.02}$ (Fig. 8-middle). As for PI, a large scatter in $P F$ (of $\sim 2$ orders of magnitude) is observed for a given value of $I$, with $P F$ values as high as $6 \%$ for $N_{\mathrm{H}_{2}} \sim 10^{23} \mathrm{~cm}^{-2}$ (see also, Fig. 20-top). This indicates that the dust grains remain well-aligned with the B-field to the highest observed column densities.

- The observed slope between the $P F$ and $I$ values (see previous paragraph) is shallower than the -1 slope expected from the progressive loss of grain alignment due to the attenuation of the interstellar radiation field with increasing column density (i.e., from the RAT theory) and shallower than the -0.5 slope expected from a purely turbulent B-field (see Fig. 20-top). In Sect.6.3, we suggest that the large $P F$ values observed towards NGC 6334 may be tracing ongoing feedback effects on the observed polarized dust emission that may result from the combination of 1) an enhanced radiation field from the newborn (high-mass) stars, 2) the re-organization of the structure of the B-field on the scale of $\mathrm{H}$ II regions (within compressed shells), and 3) the increase of the B-field strength ordering its structure and reducing depolarization due to the integration of emission along the LOS and within the beam.

- The ridge and the clump-hub structure are surrounded by sub-filaments (Fig. 10). In Sect.4.2, we investigate the variation of the polarization and physical properties along the sub-filaments from their outer (out) to their inner (in) parts. In the outer parts, the POS B-field show mostly perpendicular or random orientation with respect to the sub-filament crests. In their inner parts, where the sub-filaments are merging with the clump-hub, the B-field is parallel to their crests (cf., Fig. 13). This change of relative orientation along the sub-filaments may be due to material flowing along their crests onto the ridge and hubs. These flows of matter may drag the field lines, aligning them with the filament crests (c.f., Sect. 6.2). The polarization fractions and the dispersion of the POS B-field angle are smaller on average in the inner parts of the sub-filaments compared to their outer parts (cf., Fig. 14).

- We also observe a variation of the stability of these subfilaments (cf., Fig. 15). The kinetic virial parameter $\alpha_{\text {vir }}^{v}$ is on average smaller in the inner parts compared to the outer parts, while the magnetic virial parameter $\alpha_{\text {vir }}^{B}$ increases (by a factor of $\sim 1.8$ on average) from out to in. In the outer parts, $\alpha_{\text {vir }}^{B} \sim 0.5 \pm 0.3<1$ suggesting that the magnetic tension alone is not enough to balance gravity while the inner parts of the sub-filaments seem to be in a magnetic critical balance with $\alpha_{\text {vir }}^{B} \sim 1$. When both the magnetic and kinetic (thermal and turbulent) supports are combined, the estimated $\alpha_{\text {vir }}^{v, B} \gtrsim 1$ values suggest that the sub-filaments are in an overall balance between the effective pressure forces and gravity (Sect. 4.2). This behaviour is also seen along the ridge (Sect. 4.1).

- We identify a signature in the polarized emission towards two sub-filaments (with two spatially separated crests) that seem to be merging (as seen in dust continuum) to form a filament with a single crest (Fig. 18). We see the gradual increase of the total intensity due to the merging of the two sub-filaments and detect a drop in the polarized intensity towards the crest of the merged filaments. We suggest that this drop in $P I$ (and $P F$ ), while $I$ increases, is due to the depolarization from the integration along the LOS and within the beam of the unresolved B-field structures resulting from the merging of two different (partly independent) B-field structures along each of the initially separated filaments. This increase in $I$ towards the crests of the merged-filaments is larger than a simple addition of the intensity of the two separated-filaments, suggesting efficient infall of matter onto the merged-crest. This behaviour may be indicative of an early stage of a core-hub formation. 
- We compare the properties observed towards NGC 6334 with two other regions observed as part of the BISTRO survey: Oph-A and NGC 1333 (Fig. 17). While these three regions have physical sizes that span 2 orders of magnitude in spatial scales, from $\sim 0.2 \mathrm{pc}$ to $\sim 10 \mathrm{pc}$, they all show a similar scatter of $P F$ values, of more than 2 orders of magnitude in $P F$ (from $\sim 0.2 \%$ to $\sim 20 \%$ ). This scatter is also observed for each $I$ value, where a large scatter in $I$ is also observed for a given value of $P F$. These observed similarities suggest that the self-similar description of the physical processes in a possibly fractal magnetized ISM also has an imprint in the dust polarized thermal emission (c.f., Sect. 6.1).

- We present the first power spectrum of the POS B-field angles observed along the NGC 6334 ridge crest and compare it to the power spectrum of the intensity emission (cf., Fig. 16 and Sect. 5). The former is well represented by a power law function with a slope $\sim 20 \%$ shallower than that of the latter. We compare this result with the power spectrum of filaments obtained from an MHD numerical simulation of massive filament formation (Fig. 21) and find consistent values. These preliminary results suggest that the structure of the column densities (line mass) and POS B-field angles along the NGC 6334 filament may be each inherited from the filament formation process and have a coupled evolution (c.f., Sect. 6.4). Understanding the theoretical link between the power spectra of column density and magnetic field (POS angle and strength) may lead to a new and independent method to infer the B-field strength from polarization observations.

In this paper we have presented a first look at these rich BISTRO observations towards NGC 6334 . The different topics addressed in Sect. 6 will be investigated in dedicated and in-depth forthcoming studies (e.g., Tahani et al. in prep.). To assess the reliability of the scenario presented above regarding the change of the properties along the sub-filaments and towards the clump-hub, large-scale molecular line mapping (at the appropriate resolutions) will be needed, to better understand the dynamics of these structures. The combination of the B-field structure observed at the BISTRO scale with that at higher angular resolution will also be very valuable to study the role of the B-field in the matter assembly and fragmentation processes that lead to the formation of massive stars. Quantitative comparisons with dedicated MHD numerical simulations will also be important to understand the physical processes at play in theses high-mass star-forming hubfilament systems.

Acknowledgements. D.A. acknowledges support by FCT/MCTES through national funds (PIDDAC) by the grants UID/FIS/04434/2019 \& UIDB/04434/2020. This research is partially supported by Grants-in-Aid for Scientific Researches from the Japan Society for Promotion of Science (KAKENHI 19H0193810). C.L.H.H. acknowledges the support of the NAOJ Fellowship and JSPS KAKENHI grants 18K13586 and 20K14527. P.M.K. acknowledges support from the Ministry of Science and Technology (MOST) grants MOST 108-2112-M-001-012 and MOST 109-2112-M-001-022 in Taiwan, and from an Academia Sinica Career Development Award. C.W.L. is supported by the Basic Science Research Program through the National Research Foundation of Korea (NRF) funded by the Ministry of Education, Science and Technology (NRF-2019R1A2C1010851). W.K. is supported by the New Faculty Startup Fund from Seoul National University and by the Basic Science Research Program through the National Research Foundation of Korea (NRF-2016R1C1B2013642). This program is part of the JCMT BISTRO Large Program observed under project code M17BL011. Team BISTRO-J is in part financially supported by 260 individuals. The James Clerk Maxwell Telescope is operated by the East Asian Observatory on behalf of The National Astronomical Observatory of Japan; Academia Sinica Institute of Astronomy and Astrophysics; the Korea Astronomy and Space Science Institute; Center for Astronomical Mega-Science (as well as the National Key R\&D Program of China with No. 2017YFA0402700). Additional funding support is provided by the Science and Technology Facilities Council of the United Kingdom and participating universities in the United Kingdom, Canada, and Ireland. Additional funds for the construction of SCUBA- 2 were provided by the Canada Foundation for Innovation. The authors wish to recognize and acknowledge the very significant cultural role and reverence that the summit of Maunakea has always had within the indigenous Hawaiian community. We are most fortunate to have the opportunity to conduct observations from this mountain.

\section{References}

Andersson, B. G. 2015, Interstellar Grain Alignment: Observational Status, ed. A. Lazarian, E. M. de Gouveia Dal Pino, \& C. Melioli, Vol. 407, 59

Andersson, B. G., Lazarian, A., \& Vaillancourt, J. E. 2015, ARA\&A, 53, 501

André, P., Arzoumanian, D., Könyves, V., Shimajiri, Y., \& Palmeirim, P. 2019, A\&A, 629, L4

André, P., Di Francesco, J., Ward-Thompson, D., et al. 2014, Protostars and Planets VI, 27

André, P., Men'shchikov, A., Bontemps, S., et al. 2010, A\&A, 518, L102

André, P., Revéret, V., Könyves, V., et al. 2016, A\&A, 592, A54

Arzoumanian, D., André, P., Didelon, P., et al. 2011, A\&A, 529, L6

Arzoumanian, D., André, P., Könyves, V., et al. 2019, A\&A, 621, A42

Arzoumanian, D., André, P., Peretto, N., \& Könyves, V. 2013, A\&A, 553, A119

Arzoumanian, D., Shimajiri, Y., Inutsuka, S.-i., Inoue, T., \& Tachihara, K. 2018, PASJ, 70, 96

Arzoumanian, D., Shimajiri, Y., Roy, A., et al. 2017, Memorie della Societa Astronomica Italiana, 88, 720

Bastien, P., Bissonnette, E., Simon, A., et al. 2011, in Astronomical Society of the Pacific Conference Series, Vol. 449, Astronomical Polarimetry 2008: Science from Small to Large Telescopes, ed. P. Bastien, N. Manset, D. P. Clemens, \& N. St-Louis, 68

Bonne, L., Bontemps, S., Schneider, N., et al. 2020, A\&A, 644, A27

Chandrasekhar, S. \& Fermi, E. 1953, ApJ, 118, 113

Chapin, E. L., Berry, D. S., Gibb, A. G., et al. 2013, MNRAS, 430, 2545

Chen, H.-R., Rao, R., Wilner, D. J., \& Liu, S.-Y. 2012, ApJ, 751, L13

Chen, H.-R. V., Zhang, Q., Wright, M. C. H., et al. 2019, ApJ, 875, 24

Chibueze, J. O., Omodaka, T., Handa, T., et al. 2014, ApJ, 784, 114

Cho, J. \& Lazarian, A. 2005, ApJ, 631, 361

Coudé, S., Bastien, P., Houde, M., et al. 2019, ApJ, 877, 88

Cox, N. L. J., Arzoumanian, D., André, P., et al. 2016, A\&A, 590, A110

Crutcher, R. M. 2012, ARA\&A, 50, 29

Crutcher, R. M., Nutter, D. J., Ward-Thompson, D., \& Kirk, J. M. 2004, ApJ, 600,279

Currie, M. J., Berry, D. S., Jenness, T., et al. 2014, in Astronomical Society of the Pacific Conference Series, Vol. 485, Astronomical Data Analysis Software and Systems XXIII, ed. N. Manset \& P. Forshay, 391

Davis, Jr., L. \& Greenstein, J. L. 1951, ApJ, 114, 206

Dempsey, J. T., , P., Jenness, T., et al. 2013, MNRAS, 430, 2534

Doi, Y., Hasegawa, T., Furuya, R. S., et al. 2020, ApJ, 899, 28

Falceta-Gonçalves, D., Lazarian, A., \& Kowal, G. 2008, ApJ, 679, 537

Falceta-Gonçalves, D., Lazarian, A., \& Kowal, G. 2009, in Revista Mexicana de Astronomia y Astrofisica Conference Series, Vol. 36, 37-44

Fiege, J. D. \& Pudritz, R. E. 2000, MNRAS, 311, 85

Fischera, J. \& Martin, P. G. 2012, A\&A, 542, A77

Friberg, P., Bastien, P., Berry, D., et al. 2016, in Society of Photo-Optical Instrumentation Engineers (SPIE) Conference Series, Vol. 9914, Proc. SPIE, 991403

Fukui, Y., Kohno, M., Yokoyama, K., et al. 2018, PASJ, 70, S41

Gómez, G. C., Vázquez-Semadeni, E., \& Zamora-Avilés, M. 2018, MNRAS, 480, 2939

Hacar, A., Tafalla, M., Forbrich, J., et al. 2018, A\&A, 610, A77

Hacar, A., Tafalla, M., Kauffmann, J., \& Kovács, A. 2013, A\&A, 554, A55

Heitsch, F., Zweibel, E. G., Mac Low, M.-M., Li, P., \& Norman, M. L. 2001, ApJ, 561, 800

Hennebelle, P. \& Inutsuka, S.-i. 2019, Frontiers in Astronomy and Space Sciences, 6,5

Hennemann, M., Motte, F., Schneider, N., et al. 2012, A\&A, 543, L3

Hildebrand, R. H. 1983, QJRAS, 24, 267

Hildebrand, R. H. 1988, QJRAS, 29, 327

Hildebrand, R. H., Davidson, J. A., Dotson, J. L., et al. 2000, PASP, 112, 1215

Hill, T., Motte, F., Didelon, P., et al. 2011, A\&A, 533, A94

Hoang, T. \& Lazarian, A. 2014, MNRAS, 438, 680

Hoang, T. \& Lazarian, A. 2016, ApJ, 831, 159

Hoang, T., Tram, L. N., Lee, H., \& Ahn, S.-H. 2019, Nature Astronomy, 3, 766

Hoang, T., Tram, L. N., Lee, H., Diep, P. N., \& Bich Ngoc, N. 2020, arXiv eprints, arXiv:2010.07742

Holland, W. S., Bintley, D., Chapin, E. L., et al. 2013, MNRAS, 430, 2513 
Hull, C. L. H. \& Plambeck, R. L. 2015, Journal of Astronomical Instrumentation, 4, 1550005

Inoue, T. \& Fukui, Y. 2013, ApJ, 774, L31

Inoue, T., Hennebelle, P., Fukui, Y., et al. 2018, PASJ, 70, S53

Inutsuka, S. \& Miyama, S. M. 1997, ApJ, 480, 681

Inutsuka, S.-i. 2001, ApJl, 559, L149

Jackson, J. M., Rathborne, J. M., Foster, J. B., et al. 2013, PASA, 30, e057

Jones, R. V. \& Spitzer, Jr., L. 1967, ApJ, 147, 943

Jones, T. J., Bagley, M., Krejny, M., Andersson, B.-G., \& Bastien, P. 2015, AJ, 149,31

Jones, T. J., Klebe, D., \& Dickey, J. M. 1992, ApJ, 389, 602

Juárez, C., Girart, J. M., Zamora-Avilés, M., et al. 2017, ApJ, 844, 44

Kainulainen, J., Stutz, A. M., Stanke, T., et al. 2017, A\&A, 600, A141

Kauffmann, J., Bertoldi, F., Bourke, T. L., Evans, II, N. J., \& Lee, C. W. 2008, A\&A, 487, 993

Keown, J., Di Francesco, J., Rosolowsky, E., et al. 2019, ApJ, 884, 4

Kirk, H., Myers, P. C., Bourke, T. L., et al. 2013a, ApJ, 766, 115

Kirk, J. M., Ward-Thompson, D., Palmeirim, P., et al. 2013b, MNRAS, 432, 1424

Koch, E. W. \& Rosolowsky, E. W. 2015, MNRAS, 452, 3435

Koch, P. M., Tang, Y.-W., Ho, P. T. P., et al. 2018, ApJ, 855, 39

Könyves, V., André, P., Arzoumanian, D., et al. 2020, A\&A, 635, A34

Könyves, V., André, P., Men'shchikov, A., et al. 2015, A\&A, 584, A91

Kraemer, K. E. \& Jackson, J. M. 1999, ApJS, 124, 439

Kumar, M. S. N., Palmeirim, P., Arzoumanian, D., \& Inutsuka, S. I. 2020, A\&A, 642, A87

Kwon, J., Doi, Y., Tamura, M., et al. 2018, ApJ, 859, 4

Lazarian, A. 2007, J. Quant. Spectr. Rad. Transf., 106, 225

Lazarian, A., Andersson, B. G., \& Hoang, T. 2015, Grain alignment: Role of radiative torques and paramagnetic relaxation, 81

Lazarian, A., Goodman, A. A., \& Myers, P. C. 1997, ApJ, 490, 273

Lazarian, A. \& Hoang, T. 2007, MNRAS, 378, 910

Lee, H., Hoang, T., Le, N., \& Cho, J. 2020, ApJ, 896, 44

Lee, H. M. \& Draine, B. T. 1985, ApJ, 290, 211

Li, G.-X. \& Burkert, A. 2018, MNRAS, 474, 2167

Li, H., Griffin, G. S., Krejny, M., et al. 2006, ApJ, 648, 340

Li, H.-B., Yuen, K. H., Otto, F., et al. 2015, Nature, 520, 518

Matthews, H. E., McCutcheon, W. H., Kirk, H., White, G. J., \& Cohen, M. 2008, AJ, 136, 2083

McCutcheon, W. H., Sandell, G., Matthews, H. E., et al. 2000, MNRAS, 316, 152

Misugi, Y., Inutsuka, S.-i., \& Arzoumanian, D. 2019, ApJ, 881, 11

Molinari, S., Swinyard, B., Bally, J., et al. 2010, A\&A, 518, L100

Montier, L., Plaszczynski, S., Levrier, F., et al. 2015, A\&A, 574, A136

Motte, F., Zavagno, A., Bontemps, S., et al. 2010, A\&A, 518, L77

Muñoz, D. J., Mardones, D., Garay, G., et al. 2007, ApJ, 668, 906

Myers, P. C. 2009, ApJ, 700, 1609

Ossenkopf, V. \& Henning, T. 1994, A\&A, 291, 943

Ostriker, E. C., Stone, J. M., \& Gammie, C. F. 2001, ApJ, 546, 980

Ostriker, J. 1964, ApJ, 140, 1056

Palmeirim, P., André, P., Kirk, J., et al. 2013, A\&A, 550, A38

Pattle, K., Lai, S.-P., Hasegawa, T., et al. 2019, ApJ, 880, 27

Peretto, N., Fuller, G. A., André, P., et al. 2014, A\&A, 561, A83

Peretto, N., Fuller, G. A., Duarte-Cabral, A., et al. 2013, A\&A, 555, A112

Persi, P. \& Tapia, M. 2008, Star Formation in NGC 6334, ed. B. Reipurth, Vol. 5, 456

Persi, P. \& Tapia, M. 2010, Mem. Soc. Astron. Italiana, 81, 171

Pillai, T. G. S., Clemens, D. P., Reissl, S., et al. 2020, Nature Astronomy [arXiv:2009.14100]

Planck Collaboration Int. XIX. 2015, A\&A, 576, A104

Planck Collaboration Int. XLIV. 2016, A\&A, 596, A105

Planck Collaboration Int. XXXII. 2016, A\&A, 586, A135

Planck Collaboration Int. XXXIII. 2016, A\&A, 586, A136

Planck Collaboration Int. XXXV. 2016, A\&A, 586, A138

Planck Collaboration Int.XXXIV. 2016, A\&A, 586, A137

Plaszczynski, S., Montier, L., Levrier, F., \& Tristram, M. 2014, MNRAS, 439, 4048

Qiu, K., Wyrowski, F., Menten, K. M., et al. 2011, ApJ, 743, L25

Roy, A., André, P., Arzoumanian, D., et al. 2015, A\&A, 584, A111

Russeil, D., Schneider, N., Anderson, L. D., et al. 2013, A\&A, 554, A42

Russeil, D., Tigé, J., Adami, C., et al. 2016, A\&A, 587, A135

Sadaghiani, M., Sánchez-Monge, Á., Schilke, P., et al. 2020, A\&A, 635, A2

Sadavoy, S. I., Di Francesco, J., Johnstone, D., et al. 2013, ApJ, 767, 126

Salpeter, E. E. 1955, ApJ, 121, 161

Sandell, G. 2000, A\&A, 358, 242

Schneider, N., Csengeri, T., Bontemps, S., et al. 2010, A\&A, 520, A49+

Schneider, N., Csengeri, T., Hennemann, M., et al. 2012, A\&A, 540, L11

Shimajiri, Y., André, P., Ntormousi, E., et al. 2019, A\&A, 632, A83

Soam, A., Pattle, K., Ward-Thompson, D., et al. 2018, ApJ, 861, 65

Soler, J. D., Alves, F., Boulanger, F., et al. 2016, A\&A, 596, A93

Soler, J. D., Hennebelle, P., Martin, P. G., et al. 2013, ApJ, 774, 128
Sousbie, T. 2011, MNRAS, 414, 350

Sousbie, T., Pichon, C., \& Kawahara, H. 2011, MNRAS, 414, 384 Sugitani, K., Nakamura, F., Watanabe, M., et al. 2011, ApJ, 734, 63

Tafalla, M. \& Hacar, A. 2015, A\&A, 574, A104

Tahani, M., Plume, R., Brown, J. C., \& Kainulainen, J. 2018, A\&A, 614, A100

Tahani, M., Plume, R., Brown, J. C., Soler, J. D., \& Kainulainen, J. 2019, A\&A, 632, A68

Tang, Y.-W., Ho, P. T. P., Girart, J. M., et al. 2009, ApJ, 695, 1399

Tigé, J., Motte, F., Russeil, D., et al. 2017, A\&A, 602, A77

Tomisaka, K. 2014, ApJ, 785, 24

Vaidya, B., Hartquist, T. W., \& Falle, S. A. E. G. 2013, MNRAS, 433, 1258

Vaillancourt, J. E. 2006, PASP, 118, 1340

Voshchinnikov, N. V. 2012, J. Quant. Spectr. Rad. Transf., 113, 2334

Wang, J.-W., Koch, P. M., Galván-Madrid, R., et al. 2020, arXiv e-prints, arXiv:2011.01555

Wang, J.-W., Lai, S.-P., Eswaraiah, C., et al. 2019, ApJ, 876, 42

Wang, K., Testi, L., Ginsburg, A., et al. 2015, MNRAS, 450, 4043

Ward-Thompson, D., Pattle, K., Bastien, P., et al. 2017, ApJ, 842, 66

Whittet, D. C. B., Gerakines, P. A., Hough, J. H., \& Shenoy, S. S. 2001, ApJ, 547,872

Whittet, D. C. B., Hough, J. H., Lazarian, A., \& Hoang, T. 2008, ApJ, 674, 304

Williams, G. M., Peretto, N., Avison, A., Duarte-Cabral, A., \& Fuller, G. A. 2018, A\&A, 613, A11

Zavagno, A., André, P., Schuller, F., et al. 2020, A\&A, 638, A7

Zernickel, A., Schilke, P., \& Smith, R. J. 2013, A\&A, 554, L2

Zhang, Q., Qiu, K., Girart, J. M., et al. 2014, ApJ, 792, 116

1 Instituto de Astrofísica e Ciências do Espaço, Universidade do Porto, CAUP, Rua das Estrelas, PT4150-762 Porto, Portugal

2 Department of Physics, Graduate School of Science, Nagoya University, Furo-cho, Chikusa-ku, Nagoya 464-8602, Japan

3 Tokushima University, Minami Jousanajima-machi 1-1, Tokushima 770-8502, Japan

4 Institute of Liberal Arts and Sciences Tokushima University, Minami Jousanajima-machi 1-1, Tokushima 770-8502, Japan

5 National Astronomical Observatory of Japan, Osawa, Mitaka, Tokyo 181-8588, Japan

6 Dominion Radio Astrophysical Observatory, Herzberg Astronomy and Astrophysics Research Centre, National Research Council Canada, P. O. Box 248, Penticton, BC V2A 6J9 Canada

7 Department for Physics, Engineering Physics and Astrophysics, Queen's University, Kingston, ON, K7L 3N6, Canada

8 National Astronomical Observatory of Japan, NAOJ Chile, Alonso de Córdova 3788, Office 61B, 7630422, Vitacura, Santiago, Chile

9 Joint ALMA Observatory, Alonso de Córdova 3107, Vitacura, Santiago, Chile

10 NAOJ Fellow

11 NRC Herzberg Astronomy and Astrophysics, 5071 West Saanich Road, Victoria, BC V9E 2E7, Canada

12 Department of Physics and Astronomy, University of Victoria, Victoria, BC V8W 2Y2, Canada

13 Academia Sinica Institute of Astronomy and Astrophysics, No.1, Sec. 4., Roosevelt Road, Taipei 10617, Taiwan

14 Department of Earth Science and Astronomy, Graduate School of Arts and Sciences, The University of Tokyo, 3-8-1 Komaba, Meguro, Tokyo 153-8902, Japan

15 Korea Astronomy and Space Science Institute, 776 Daedeokdae-ro, Yuseong-gu, Daejeon 34055, Republic of Korea

16 University of Science and Technology, Korea, 217 Gajeong-ro, Yuseong-gu, Daejeon 34113, Republic of Korea

17 Department of Physics, Faculty of Science and Engineering, Meisei University, 2-1-1 Hodokubo, Hino, Tokyo 191-8506, Japan

18 Department of Astronomy, Graduate School of Science, The University of Tokyo, 7-3-1 Hongo, Bunkyo-ku, Tokyo 113-0033, Japan

19 School of Physics and Astronomy, Cardiff University, The Parade, Cardiff, CF24 3AA, UK

20 Laboratoire d'Astrophysique (AIM), CEA/DRF, CNRS, Université Paris-Saclay, Université Paris Diderot, Sorbonne Paris Cité, 91191 Gif-sur-Yvette, France

21 Centre de recherche en astrophysique du Québec \& département de physique, Université de Montréal, C.P. 6128 Succ. Centre-ville, Montréal, QC, H3C 3J7, Canada 
${ }^{22}$ East Asian Observatory, 660 N. A'ohōkū Place, University Park, Hilo, HI 96720, USA

${ }^{23}$ Institute of Astronomy and Department of Physics, National Tsing Hua University, Hsinchu 30013, Taiwan

${ }^{24}$ CAS Key Laboratory of FAST, National Astronomical Observatories, Chinese Academy of Sciences, People's Republic of China

25 National Astronomical Observatories, Chinese Academy of Sciences, A20 Datun Road, Chaoyang District, Beijing 100012, People's Republic of China

26 Nobeyama Radio Observatory, National Astronomical Observatory of Japan, National Institutes of Natural Sciences, Nobeyama, Minamimaki, Minamisaku, Nagano 384-1305, Japan

27 Department of Physics and Astronomy, University College London, WC1E 6BT London, UK

28 Department of Earth Science Education, (SNU), 1 Gwanak-ro, Gwanak-gu, Seoul 08826, Republic of Korea

29 Korea Astronomy and Space Science Institute (KASI), 776 Daedeokdae-ro, Yuseong-gu, Daejeon 34055, Republic of Korea

30 Department of Astronomy, Yunnan University, Kunming, 650091, PR China

31 Departamento de Astronomía, Universidad de Concepción, Av. Esteban Iturra s/n, Distrito Universitario, 160-C, Chile

32 Centre for Astronomy, School of Physics, National University of Ireland Galway, University Road, Galway, Ireland

33 SOFIA Science Center, Universities Space Research Association, NASA Ames Research Center, Moffett Field, California 94035, USA

34 Xinjiang Astronomical Observatory, Chinese Academy of Sciences, 830011 Urumqi, People's Republic of China

35 Jeremiah Horrocks Institute, University of Central Lancashire, Preston PR1 2HE, UK

36 School of Astronomy and Space Science, Nanjing University, 163 Xianlin Avenue, Nanjing 210023, People's Republic of China

37 Key Laboratory of Modern Astronomy and Astrophysics, Ministry of Education, Nanjing 210023, People's Republic of China

38 SKA Organisation, Jodrell Bank, Lower Withington, Macclesfield, SK11 9FT, UK

39 Jodrell Bank Centre for Astrophysics, School of Physics and Astronomy, University of Manchester, Manchester, M13 9PL, UK

${ }^{40}$ Purple Mountain Observatory, Chinese Academy of Sciences, 2 West Beijing Road, 210008 Nanjing, People's Republic of China

41 Institute of Astronomy, National Central University, Zhongli 32001, Taiwan

42 Department of Astronomy and Space Science, Chungnam National University, 99 Daehak-ro, Yuseong-gu, Daejeon 34134, Republic of Korea

43 School of Physics, Astronomy \& Mathematics, University of Hertfordshire, College Lane, Hatfield, Hertfordshire AL10 9AB, UK

44 Vietnam National Space Center, Vietnam Academy of Science and Technology, 18 Hoang Quoc Viet, Hanoi, Vietnam

45 Astrophysics Research Institute, Liverpool John Moores University, IC2, Liverpool Science Park, 146 Brownlow Hill, Liverpool, L3 5RF, UK

46 Department of Physics and Astronomy, The University of Manitoba, Winnipeg, Manitoba R3T2N2, Canada

47 Jodrell Bank Centre for Astrophysics, School of Physics and Astronomy, University of Manchester, Oxford Road, Manchester, M13 9PL, UK

48 Department of Physics, The Chinese University of Hong Kong, Shatin, N.T., Hong Kong

49 Physics and Astronomy, University of Exeter, Stocker Road, Exeter EX4 4QL, UK

50 Subaru Telescope, National Astronomical Observatory of Japan, 650 N. A'ohōkū Place, Hilo, HI 96720, USA

51 Department of Physics and Astronomy, The University of Western Ontario, 1151 Richmond Street, London N6A 3K7, Canada

52 Hiroshima Astrophysical Science Center, Hiroshima University, Kagamiyama 1-3-1, Higashi-Hiroshima, Hiroshima 739-8526, Japan
53 Department of Physics, Hiroshima University, Kagamiyama 1-3-1, Higashi-Hiroshima, Hiroshima 739-8526, Japan

${ }^{54}$ Core Research for Energetic Universe (CORE-U), Hiroshima University, Kagamiyama 1-3-1, Higashi-Hiroshima, Hiroshima 7398526, Japan

55 European Southern Observatory, Karl-Schwarzschild-Str. 2, 85748 Garching, Germany

56 Astronomical Institute, Graduate School of Science, Tohoku University, Aoba-ku, Sendai, Miyagi 980-8578, Japan

57 Department of Physics and Astronomy, McMaster University, Hamilton, ON L8S 4M1 Canada

58 Department of Physics and Atmospheric Science, Dalhousie University, Halifax B3H 4R2, Canada

59 Department of Space, Earth \& Environment, Chalmers University of Technology, SE-412 96 Gothenburg, Sweden

60 School of Space Research, Kyung Hee University, 1732 Deogyeongdaero, Giheung-gu, Yongin-si, Gyeonggi-do 17104, Republic of Korea

61 CAS Key Laboratory of FAST, National Astronomical Observatories, Chinese Academy of Sciences, People's Republic of China; University of Chinese Academy of Sciences, Beijing 100049, People's Republic of China

62 Xinjiang Astronomical Observatory, Chinese Academy of Sciences, 150 Science 1-Street, Urumqi 830011, Xinjiang, People's Republic of China

63 School of Astronomy and Space Science, Nanjing University, 163 Xianlin Avenue, Nanjing 210023, People's Republic of China

64 Key Laboratory of Modern Astronomy and Astrophysics, Nanjing University, Ministry of Education, Nanjing 210023, People's Republic of China

65 Key Laboratory for Research in Galaxies and Cosmology, Shanghai Astronomical Observatory, Chinese Academy of Sciences, 80 Nandan Road, Shanghai 200030, People's Republic of China

66 Faculty of Education \& Center for Educational Development and Support, Kagawa University, Saiwai-cho 1-1, Takamatsu, Kagawa, 760-8522, Japan

67 Department of Astronomy, Graduate School of Science, Kyoto University, Sakyo-ku, Kyoto 606-8502, Japan

68 SOKENDAI (The Graduate University for Advanced Studies), Hayama, Kanagawa 240-0193, Japan

69 Department of Physics and Astronomy, Graduate School of Science and Engineering, Kagoshima University, 1-21-35 Korimoto, Kagoshima, Kagoshima 890-0065, Japan

70 Astrophysics Group, Cavendish Laboratory, J. J. Thomson Avenue, Cambridge CB3 0HE, UK

71 Kavli Institute for Cosmology, Institute of Astronomy, University of Cambridge, Madingley Road, Cambridge, CB3 0HA, UK

72 Faculty of Pure and Applied Sciences, University of Tsukuba, 1-1-1 Tennodai, Tsukuba, Ibaraki 305-8577, Japan

73 Department of Physics, School of Science and Technology, Kwansei Gakuin University, 2-1 Gakuen, Sanda, Hyogo 669-1337, Japan

74 Astrobiology Center, National Institutes of Natural Sciences, 2-21-1 Osawa, Mitaka, Tokyo 181-8588, Japan

75 University of Science and Technology of Hanoi, Vietnam Academy of Science and Technology, 18 Hoang Quoc Viet, Hanoi, Vietnam

76 Jet Propulsion Laboratory, M/S 169-506, 4800 Oak Grove Drive, Pasadena, CA 91109, USA

77 University of South Wales, Pontypridd, CF37 1DL, UK

78 Department of Applied Mathematics, University of Leeds, Woodhouse Lane, Leeds LS2 9JT, UK

79 National Radio Astronomy Observatory, 520 Edgemont Road, Charlottesville, VA 22903, USA

80 Univ. Grenoble Alpes, CNRS, IPAG, 38000 Grenoble, France

81 School of Physics and Astronomy, University of Leeds, Woodhouse Lane, Leeds LS2 9JT, UK 


\section{Appendix A: Details on the quality assessments of the BISTRO data of NGC 6334}

To complement the presentation of the data of the JCMT/SCUBA-2/POL-2 BISTRO observations at $850 \mu \mathrm{m}$ towards the NGC 6334 field (see Sect.2), we here present maps of the uncertainties of the total intensity $I$, the polarized intensity $P I$, polarization fraction $P F$, and POS B-field angle. These maps show the statistical uncertainties derived from the pol2map data reduction pipeline reprojected onto $12^{\prime \prime}$-pixel size grids (cf., http://starlink.eao.hawaii.edu/ docs/sc22.htx/sc22.html). Two DAISY maps have been observed towards the North and the South of the field and combined into $\mathrm{a} \sim 10 \mathrm{pc} \times 2 \mathrm{pc}$ mosaic. The limits of the two sub-fields are indicated on the top-right hand side panel of Fig. A.1. The 6'-diameter circles indicate the area where the observation pattern of the POL2-DAISY mode yields uniform noise coverage. Towards these areas of the map, the data have the lowest uncertainties (see Fig. A.1). The $I$ map shows larger uncertainties towards the bright compact sources due to the increase of pointing and calibration uncertainties towards steep intensity gradients. We see larger uncertainties towards the edge of the maps and in the regions between the two sub-fields. These larger uncertainties on $I(\delta I)$ can be seen on the right-hand side panel of Fig. A.2, which shows a scatter plot of $\delta I$ as a function of $I$. For $S N R(I)>3, \delta I$ is mostly uniform with a mean value of $\sim 1.6 \mathrm{mJy} / 14^{\prime \prime}$-beam. We see larger $\delta I$ for the extreme $I$ values $\gtrsim 8 \mathrm{Jy} / 14^{\prime \prime}$-beam towards the bright compact sources (c.f., Fig. A.1). More than $65 \%$ and $70 \%$ (2293 pixels) of the emission for $S N R(I)>3$ and $S N R(I)>5$, respectively, correspond to $S N R(I)>25$. For $S N R(I)>25$ (2293 pixels), $79 \%$ of the data points (1822 pixels) have $S N R(P I)>3$. Only $4 \%$ (81 pixels) of the total pixels with $S N R(P I)>3$ have $S N R(I)<25$. This excellent overall SNR can be also seen on Fig. A.3 showing SNR contours over-plotted on the $I$ and $P I$ maps. For the analysis presented in this paper, we thus select data points with $\operatorname{SNR}(I)>25$ that correspond also mostly to data points with $S N R(P I)>3$. The selection criteria on $S N R(P I)$ is defined by the debiasing method that we use and which is shown to be reliable for $S N R(P I)>3$ (c.f., 2.2, Vaillancourt 2006; Plaszczynski et al. 2014).

Figure A. 4 shows the distribution of the observed Stokes parameters for $S N R(I)>25$. The total intensity spans $\sim 3$ orders of magnitude showing the large intensity dynamic range of these observations from $N_{\mathrm{H}_{2}} \sim 10^{21} \mathrm{~cm}^{-2}$ to $\sim 10^{24} \mathrm{~cm}^{-2}$. The Stokes $Q$ and $U$ values have asymmetric distributions about 0 with different shapes. The $U$ distribution is skewed towards positive values. This distribution is compatible with a dominant POS B-field mostly at $45^{\circ}+90^{\circ}=135^{\circ}$ with respect to the North direction (a peak in the histogram of $\chi_{B_{\mathrm{POS}}}$ can be seen at $\sim 130^{\circ}$, c.f, Fig. 6). The mean and dispersion of the uncertainties of the $I, Q$, and $U$ Stokes parameters are $\delta I=(1.6 \pm 1.8) \mathrm{mJy} / 14^{\prime \prime}$-beam, $\delta Q=$ $(1.13 \pm 0.29) \mathrm{mJy} / 14^{\prime \prime}$-beam, and $\delta U=(1.6 \pm 0.30) \mathrm{mJy} / 14^{\prime \prime}-$ beam (see Fig. A.4-right).

Figure A. 5 shows the distributions of the uncertainties of $P I$, $P F$, and $\chi_{B_{\mathrm{POS}}}$ for $S N R(I)>25$ and $S N R(P I)>3$. The mean and dispersion of the uncertainties of these distributions are $\delta P I=$ $(1.1 \pm 0.3) \mathrm{mJy} / 14^{\prime \prime}$-beam, $\delta P F=(0.2 \pm 0.3) \mathrm{mJy} / 14^{\prime \prime}$-beam, and $\delta \chi_{B_{\mathrm{POS}}}=(7 \pm 1)^{\circ},(4 \pm 1)^{\circ}$, and $(1.3 \pm 0.8)^{\circ}$ for data points with $3<$ $S N R(P I)<5,5<S N R(P I)<10$, and $S N R(P I)>10$, respectively.

\section{Appendix B: Column density and dust temperature maps of the NGC 6334 filament network}

\section{Appendix B.1: Column density and dust temperature}

We derive the column density $\left(N_{\mathrm{H}_{2}}\right)$ map of the NGC 6334 field observed by BISTRO from the total intensity Stokes I map, with the relation

$N_{\mathrm{H}_{2}}=I_{850} /\left(B_{850}[T] \kappa_{850} \mu_{\mathrm{H}_{2}} m_{\mathrm{H}}\right)$,

where $\kappa_{v}^{850}=0.0182 \mathrm{~cm}^{2} / \mathrm{g}$ is the dust opacity per unit mass of dust + gas at $850 \mu \mathrm{m}$ (e.g., Ossenkopf \& Henning 1994), $\mu_{\mathrm{H}_{2}}=$ 2.8 is the mean molecular weight per hydrogen molecule (e.g., Kauffmann et al. 2008), $m_{\mathrm{H}}$ is the mass of a hydrogen atom, and $B_{850}[T]$ is the Planck function for a given temperature $T$.

Figure B.1-left, shows the dust temperature map of this region derived from grey-body fits to the Herschel five wavelength data presented in Russeil et al. (2013). As can be seen in this latter figure, in the northeast section of the NGC 6334 filamentary structure (e.g., crests 4, 5, 6, and 7), which is the least affected by the heating from the multiple $\mathrm{H}$ II bubbles present in the field (Russeil et al. 2016), the dust temperature is $\mathrm{T}_{\text {dust }} \sim 20 \mathrm{~K}$. Towards the Southern parts, the dust temperatures derived from the LOS integrated Herschel emission reach values up to $\sim 30 \mathrm{~K}$, which possibly trace the emission of warmer dust grains located mostly in the outer layers of the cloud. The dust temperatures $(>25 \mathrm{~K})$ derived from Herschel data in these regions may not provide a good estimate of the gas temperature of the cold and dense molecular cloud traced with the $850 \mu \mathrm{m}$ emission of the BISTRO data.

We thus derive a column density map, $N_{\mathrm{H}_{2}}^{\mathrm{T}=20 \mathrm{~K}}$, for a constant $T=20 \mathrm{~K}$ corresponding to the dominent temperature observed towards the Northern part of the NGC 6334 molecular cloud that is less affected by the warm $\mathrm{H}$ II regions. Temperatures of about $20 \mathrm{~K}$ are also compatible with the kinetic temperature toward star forming high-mass filaments and hubs derived from $\mathrm{NH}_{3} \mathrm{GBT}$ observations of the KEYSTONE project (Keown et al. 2019).

For completeness, we also derive a column density map, $N_{\mathrm{H}_{2}}^{\mathrm{T}_{\text {dust }}^{\text {Hershel }}}$, using the Herschel dust temperature map at a resolution of 36" (Russeil et al. 2013, and Fig. B.1-left) to compare with $N_{\mathrm{H}_{2}}^{\mathrm{T}=20 \mathrm{~K}}$. The distribution of the $N_{\mathrm{H}_{2}}^{\mathrm{T}=20 \mathrm{~K}} / N_{\mathrm{H}_{2}}^{\mathrm{T}_{\text {dust }}^{\text {Herscl }}}$ ratio of these two maps, towards the field observed by BISTRO with $S N R(I)>25$, is shown in the right panel of Fig. B.1. This distribution is narrow, with a mean of 1.32 and a standard deviation of 0.35 . The difference between the two maps, $N_{\mathrm{H}_{2}}^{\mathrm{T}=20 \mathrm{~K}}$ and $N_{\mathrm{H}_{2}}^{\mathrm{T} \text { dust }}$, of about $30 \%$ on average is mostly smaller than the dispersion of the $N_{\mathrm{H}_{2}}^{\mathrm{T}=20 \mathrm{~K}}$ found along the different crests studied in the paper, cf., column (2) of Table 2.

Thus, for the analysis presented in this paper, we derive the column density using a constant dust temperature of $20 \mathrm{~K}$.

\section{Appendix B.2: Comparison of BISTRO data with Herschel/SPIRE+ArTéMiS data}

Here we compare the BISTRO observations at $850 \mu \mathrm{m}$ with observations towards the same region obtained by Herschel/SPIRE (Motte et al. 2010) combined with ArTéMiS data at $350 \mu \mathrm{m}$ at $8^{\prime \prime}$ resolution (André et al. 2016).

To compare the two observations at different wavelengths, we estimate the column density from the total intensity Stokes $I$ values with the dust opacity per unit mass of dust + gas 

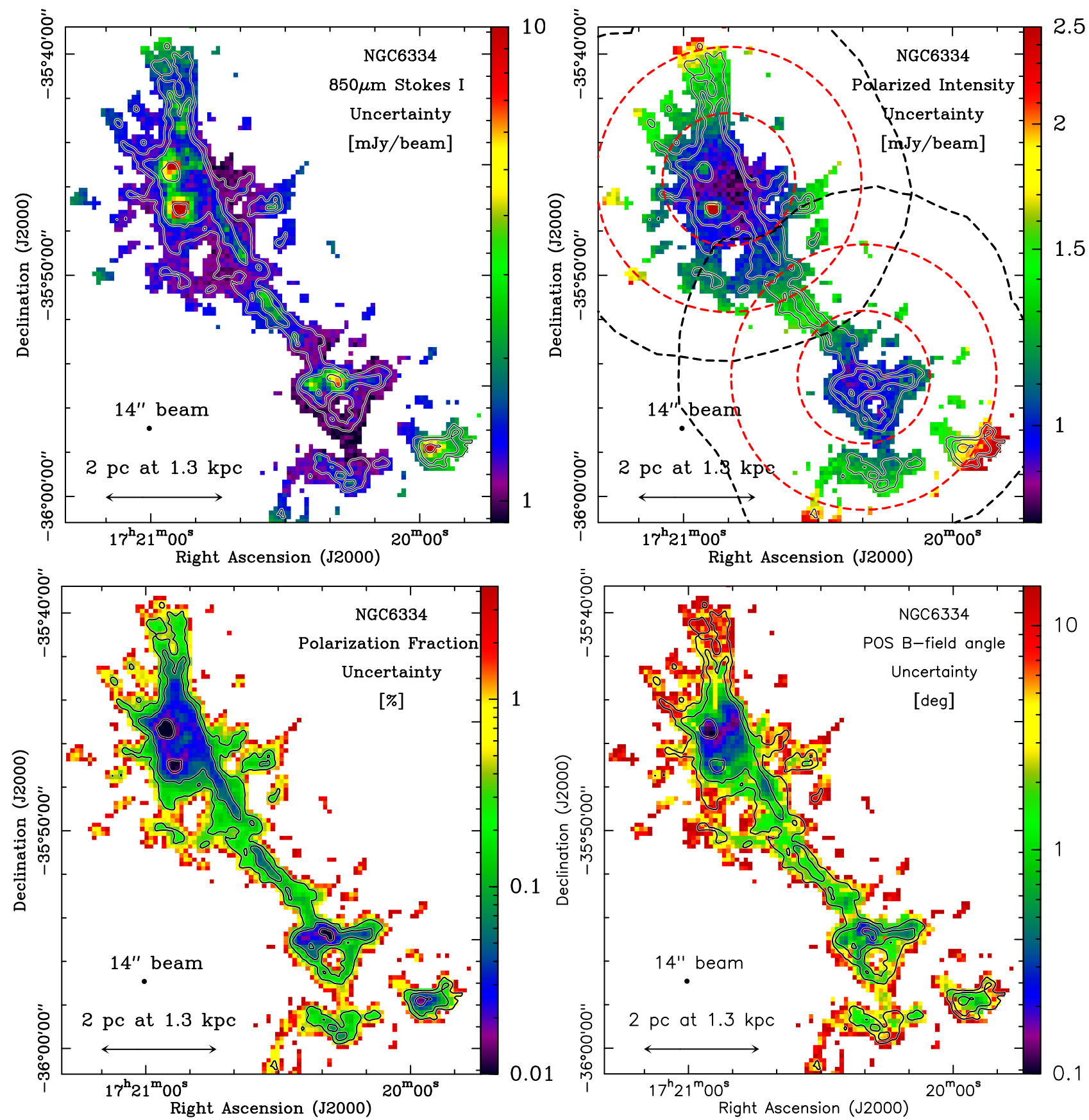

Fig. A.1. Maps showing the distribution of the uncertainties derived for Stokes $I, P I, P F$, and $\chi_{B_{\mathrm{POS}}}$ from left to right and top to bottom. The HPBW resolution of these maps is $14^{\prime \prime}$. The same emission area is plotted for all the four maps corresponding to $\operatorname{SNR}(I)>25$. The contours, which are the same for all the plots, correspond to $I=0.4,1.4$, and $8 \mathrm{Jy} \mathrm{beam}^{-1}$ or $N_{\mathrm{H}_{2}} \sim(2,6$, and 37$) \times 10^{22} \mathrm{~cm}^{-2}$ (as in Fig. 1). The black dashed circles over-plotted on the PI map (top right) indicate the limits of the two observed DAISY sub-fields centred on (17:20:50.011, -35:45:34.33) and (17:20:19.902, -35:54:30.45) in (R.A. J2000, Dec. J2000) for the North and South fields, respectively. The small and large red dashed circles have sizes of $6^{\prime}$ and $12^{\prime}$.

$\kappa_{v}^{850}=0.0182 \mathrm{~cm}^{2} / \mathrm{g}$ for the BISTRO data at $850 \mu \mathrm{m}$ and $\kappa_{v}^{350}=$ $0.1 \mathrm{~cm}^{2} / \mathrm{g}$ for the Herschel/SPIRE+ArTéMiS data at $350 \mu \mathrm{m}$ (Ossenkopf \& Henning 1994; Kauffmann et al. 2008). For both maps, we use a mean dust temperature of $T=20 \mathrm{~K}$ as explained above and also adopted by André et al. (2016).

Figure B.2 shows the column density values derived from BISTRO $850 \mu \mathrm{m}$ data and those derived from SPIRE+ArTéMiS data at $350 \mu \mathrm{m}$ data smoothed to the same resolution of $14^{\prime \prime}$. For column densities $\gtrsim 3 \times 10^{22} \mathrm{~cm}^{-2}$ both maps agree within a factor $<2$ (Fig. B.2-left).
We derive mean radial profiles perpendicular to the main axis of the elongated filament and averaged along its length. In practice, the maps have first been rotated by $30^{\circ}$ (corresponding to the mean orientation of NGC 6334 on the plane-of-thesky) from East to North. Second, horizontal cuts, perpendicular to the main axis of NGC 6334 have been averaged from South to North along the $10 \mathrm{pc}$ structure. Figure B.2-middle shows the mean radial column density profiles perpendicular to the NGC 6334 cloud. For $N_{\mathrm{H}_{2}} \approx 3 \times 10^{22} \mathrm{~cm}^{-2}$ the mean ratio between the two profiles is $\sim 1$.6. For $N_{\mathrm{H}_{2}}<3 \times 10^{22} \mathrm{~cm}^{-2}$, corresponding to scales $r \gtrsim 0.3 \mathrm{pc}$ from the central part of the filament, the ratio 

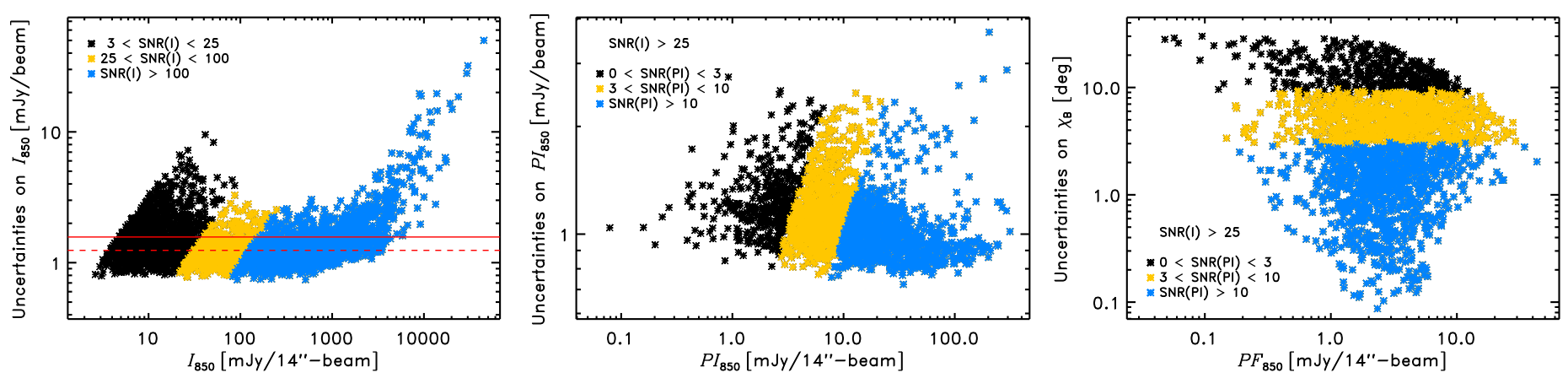

Fig. A.2. Left: Measurement uncertainties on Stokes $I$ as a function of the signal on $I$. The black, yellow, and blue data points correspond to $3<S N R(I)<25,25<S N R(I)<100$, and $S N R(I)>100$, respectively. The dashed and solid red lines show the median $(1.24 \mathrm{mJy} / \mathrm{beam})$ and mean $(1.57 \mathrm{mJy} / \mathrm{beam})$ uncertainties, respectively. Middle: Uncertainties on $P I$ as a function of $P I$ for $S N R(I)>25$. The black, yellow, and blue data points correspond to $0<S N R(P I)<3,3<S N R(P I)<10$, and $S N R(P I)>10$, respectively. Right: Uncertainties on the POS B-field angle, $\chi_{B_{\mathrm{POS}}}$, as a function of $P F$ for $S N R(I)>25$. The colours are the same as in the middle plot.
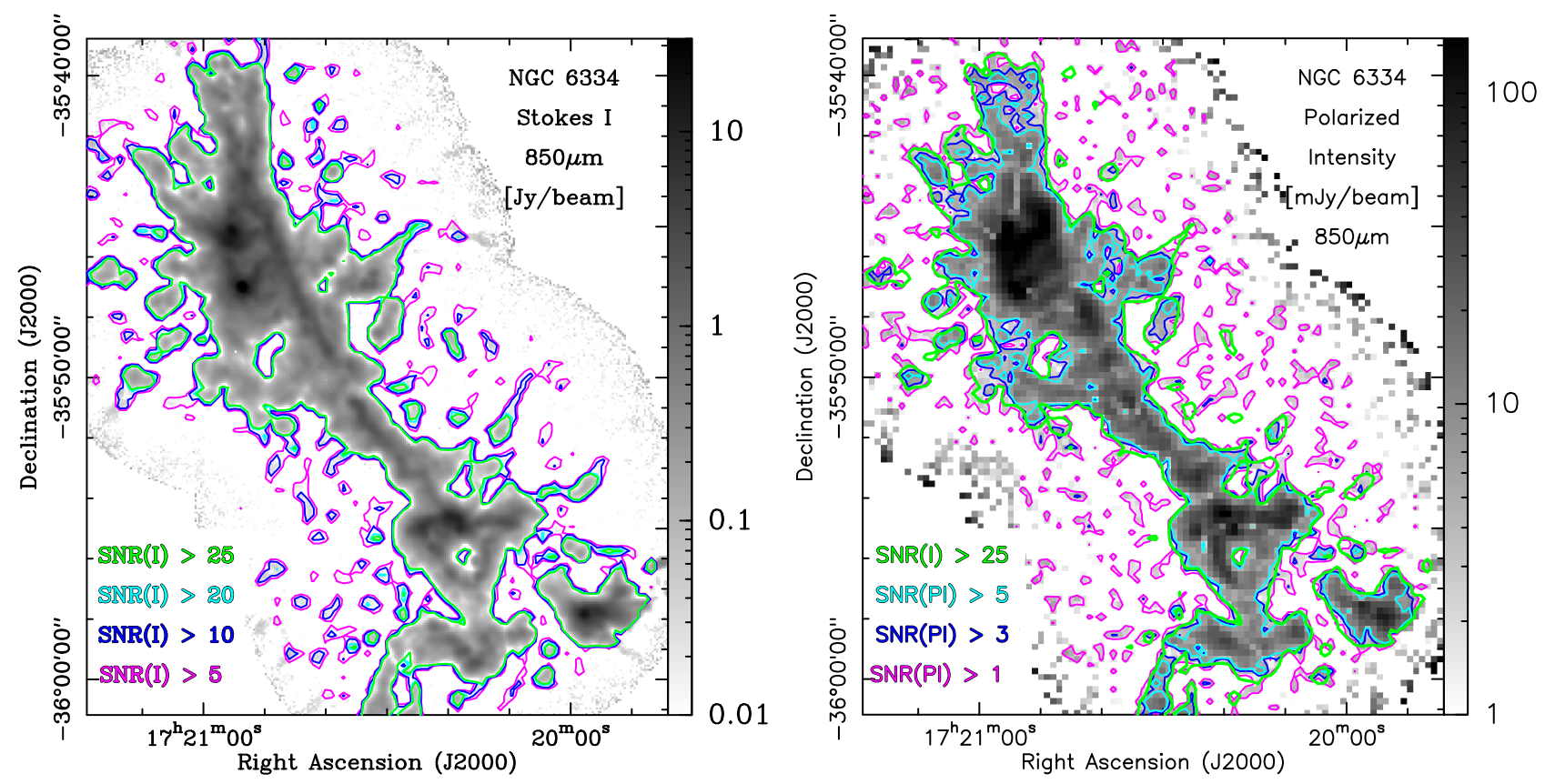

Fig. A.3. Left: Stokes $I$ map at $850 \mu$ m observed with the JCMT SCUBA-2/POL-2 towards NGC 6334. The HPBW resolution of these maps is $14^{\prime \prime}$. The pixel size of the map is $4^{\prime \prime}$. The magenta, blue, cyan, and green contours are $S N R(I)>5,10,20$, and 25 , respectively. The $S N R(I)=25$ correspond to $I \sim 30 \mathrm{mJy}_{\text {beam }}^{-1}$ or $N_{\mathrm{H}_{2}} \sim 1.5 \times 10^{21} \mathrm{~cm}^{-2}$. Right: Map of the polarized emission PI. The HPBW resolution of these maps is $14^{\prime \prime}$. The pixel size of the map is $12^{\prime \prime}$. The magenta, blue, and cyan contours are $\operatorname{SNR}(P I)>1,3$, and 5, respectively. The green contour corresponds to $\operatorname{SNR}(I)>25$ and is the same as in the left hand side panel.
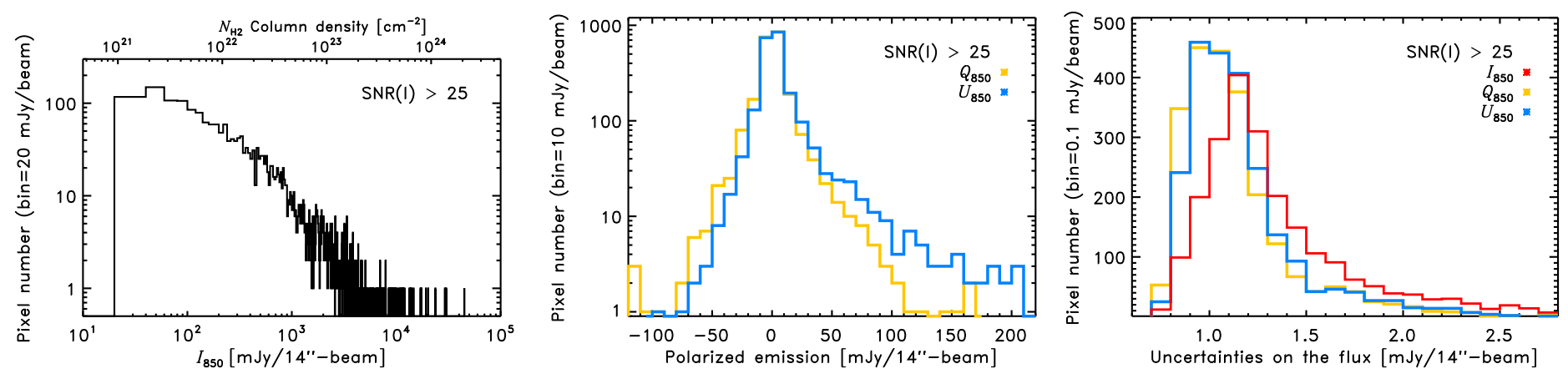

Fig. A.4. Distribution of the signal of Stokes $I$ (left), $Q$ and $U$ (middle) for $S N R(I)>25$. The measured uncertainties on the three Stokes parameters are shown on the right hand plot. 

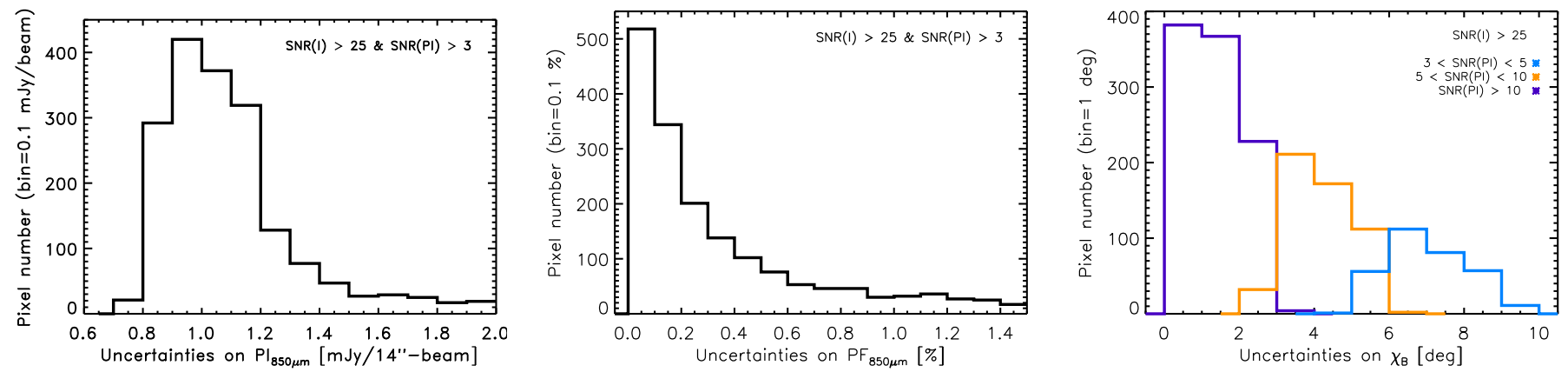

Fig. A.5. Distributions of the uncertainties on $P I$ (left), $P F$ (middle), and $\chi_{B_{\mathrm{POS}}}$ (right) for $S N R(I)>25$ and $S N R(P I)>3$ corresponding to the maps shown in Fig. A.1. On the right hand side plot the different colors are as follow: blue for $3<S N R(P I)<5$, orange for $5<S N R(P I)<10$, and purple for $S N R(P I)>10$.

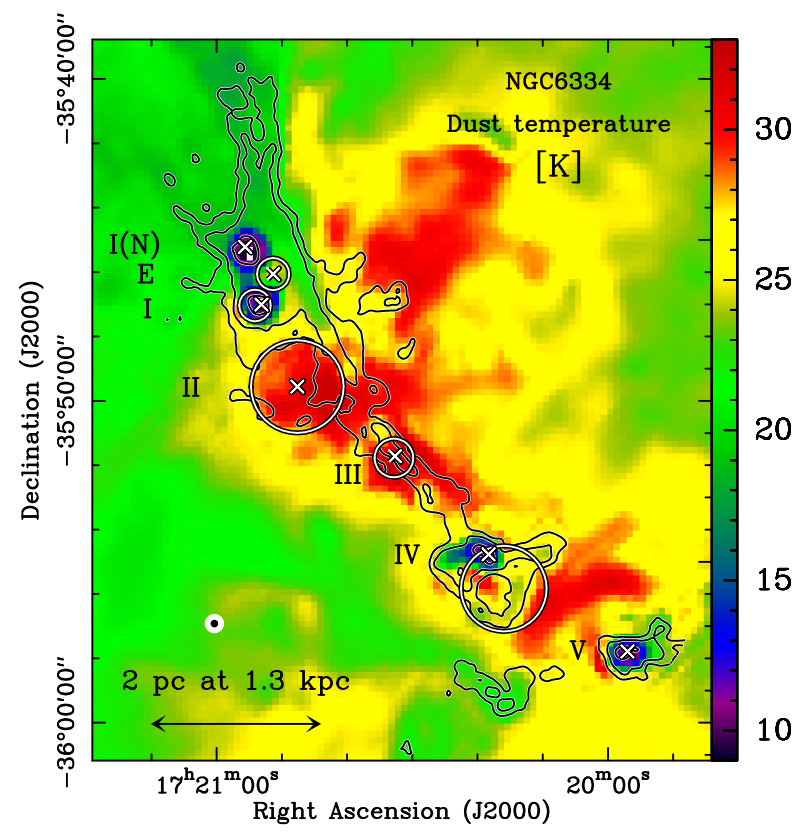

Fig. B.1. Left: Dust temperature $\mathrm{T}_{\text {dust }}^{\text {Hershel }}$ map (Russeil et al. 2013) derived from Herschel data observed as part of the HOBYS key program (Motte et al. 2010). This map is at the resolution of 36". The contours (corresponding to $I=0.4,1.4$, and $8 \mathrm{Jy} \mathrm{beam}^{-1}$ of the BISTRO data), the crosses, and the circles are the same as in Fig. 1-top-left. The Herschel (36") and BISTRO (14") beams are shown on the left bottom of the plot as concentric filled white and black circles, respectively. Right: Distribution of the ratio $N_{\mathrm{H}_{2}}^{\mathrm{T}=20 \mathrm{~K}} / N_{\mathrm{H}_{2}}^{\text {Tust }}$ (serchel Sect. B.1 describing the derivation of the two quantities). The mean value of this distribution is 1.3 , as indicated by the vertical line.

$N_{\mathrm{H}_{2}}^{\mathrm{Art}+\mathrm{SPIRE}} / N_{\mathrm{H}_{2}}^{\mathrm{BISTRO}}$ increases up to values of $\sim 10$ for $r \sim 1 \mathrm{pc}$ or $r \sim 2$.'6 (Fig. B.2-right).

The drop of the observed emission of the BISTRO data compared to the emission observed by Herschel may be due to the removal of the large scale emission affecting the JCMT ground based data while Herschel data are in principle not affected by this problem.

Since NGC 6334 is located within $0.6 \mathrm{deg}$ of the Galactic Plane, the emission is affected by large-scale "Galactic emission", which corresponds to background and foreground emission $\left(N_{\mathrm{H}_{2}} \sim 3 \times 10^{22} \mathrm{~cm}^{-2}\right)$ observed along the LOS in the surrounding of the NGC 6334 molecular cloud. We estimate here that "Galactic emission" emission to be $N_{\mathrm{H}_{2}} \sim 3 \times 10^{22} \mathrm{~cm}^{-2}$.

We hence compare the column density derived from BISTRO data with the "Galactic emission" subtracted column density values of the SPIRE+ArTéMiS data. As can be seen in Fig. B.2-middle and left, these "Galactic emission" column density subtracted SPIRE+ArTéMiS data are compatible within $\sim 50 \%$ with the column density derived from BISTRO observations at $850 \mu \mathrm{m}$ down to $N_{\mathrm{H}_{2}} \sim 10^{21} \mathrm{~cm}^{-2}$. 
We thus conclude that our BISTRO data trace the intrinsic emission of the molecular cloud of NGC 6334 filtering out the large-scale emission on the order of $N_{\mathrm{H}_{2}} \sim 3 \times 10^{22} \mathrm{~cm}^{-2}$ corresponding, in this case, to a combination of extended emission physically linked to the NGC 6334 molecular complex and to LOS foreground/background Galactic emission.

\section{Appendix C: Tests on the power spectrum analysis}

The observations show a well defined power law shape of the power spectra of $I, \chi_{B_{\text {POS }}}$, and $\phi_{\text {diff }}(\mathrm{cf} ., 16)$. To check whether such well-defined power law power spectra could be the result of uncorrelated emission, we randomly reordered the observed data along the crest of the studied filament and derived the power spectrum for 50 realisations of test-data. Figure C.1-left shows an example of the power spectrum derived from one of the realisations. These power spectra are not well represented by power laws. Figure C.1-right shows the histogram of the slopes measured by fitting the test power spectra for the 50 realisations. The derived slopes are compatible with a flat spectrum. We hence conclude that the observed power spectra with well defined power law shapes are a result of the correlated fluctuations of these properties along the filament crest. 

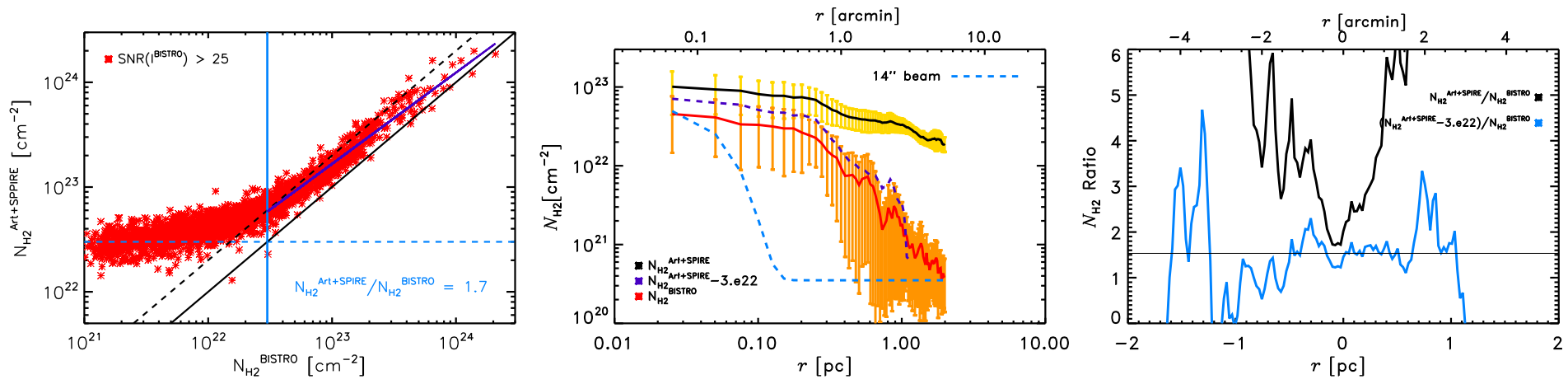

Fig. B.2. Left: Comparing the column density values derived from ArTéMiS+SPIRE ( $N_{\mathrm{H}_{2}}^{\mathrm{Art}+\mathrm{SPIRE}}$ ) at $350 \mu \mathrm{m}$ (from André et al. 2016) and BISTRO $\left(N_{\mathrm{H}_{2}}^{\mathrm{BISTOO}}\right)$ at $850 \mu \mathrm{m}$ (see Appendix B.2 for the expression used to estimate the column density from the observed total intensity). The vertical blue line indicates $N_{\mathrm{H}_{2}}=3 \times 10^{22} \mathrm{~cm}^{-2}$ above which the two maps agree within a factor $<2$. The oblique black solid and dashed lines show the $y=x$ and $y=2 x$ relations. Middle: Radial column density profiles (in log scale) perpendicular to and averaged along the 10 pc NGC 6334 elongated structure (i.e., crests from 1 to 5 in Fig. 10). The solid black and red lines correspond to the average profiles derived from ArTéMiS+SPIRE and BISTRO data, respectively. The purple dashed curve corresponds to $N_{\mathrm{H}_{2}}^{\text {Art }+ \text { SPIRE }}-N_{\mathrm{H}_{2}}^{\text {Galactic }}$, where $N_{\mathrm{H}_{2}}^{\text {Galactic }}=3 \times 10^{22} \mathrm{~cm}^{-2}$ corresponds to the large scale "Galactic emission" filtered out in the BISTRO maps. The angular resolution of the data is indicated by the blue dashed Gaussian function. Right: The mean profiles (in linear scale) of the ratios $N_{\mathrm{H}_{2}}^{\text {Art+SPIRE }} / N_{\mathrm{H}_{2}}^{\text {BISTRO }}$ (black) and $\left(N_{\mathrm{H}_{2}}^{\mathrm{Art}+\mathrm{SPIRE}}-N_{\mathrm{H}_{2}}^{\text {Galactic }}\right) / N_{\mathrm{H}_{2}}^{\text {BISTRO }}$ (blue). The mean value of this latter ratio is $\sim 1.5$ indicated by the horizontal line.
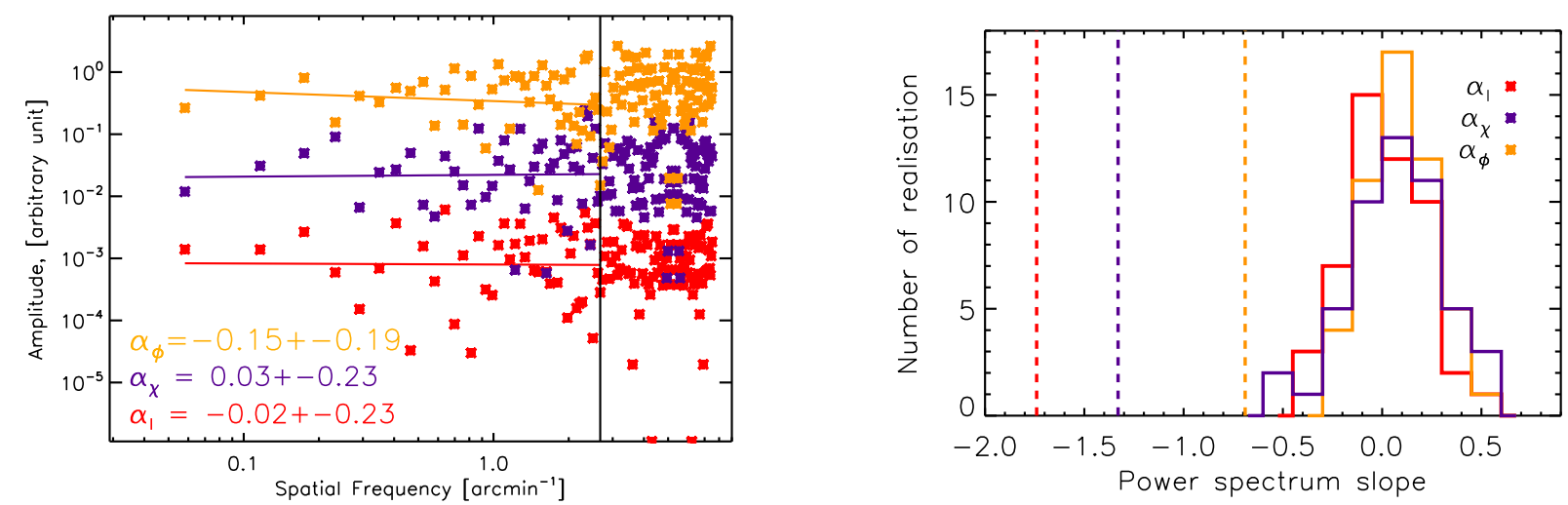

Fig. C.1. Left: A power spectrum example of the test data (intensity in red, POS B-field angle in purple, and difference angle in yellow), where the observed values along the ridge crest are randomly reordered. The vertical line shows the spatial resolution of the data. The data points have pixel size of $4 "$. The straight coloured lines show the best power-law fits to the power spectra and the derived power-law indexes $(\alpha)$ are shown on the bottom left hand side of the plot. Right: Histogram of $\alpha$ for 50 realisations of random reorganization of the observed values. The vertical dashed lines indicate the derived power spectrum slopes from the observed values (see Fig. 16). 